\title{
Calculus of functors and model categories, II
}

\author{
GEORG BIEDERMANN \\ OLIVER RÖNDIGS
}

\begin{abstract}
This is a continuation, completion, and generalization of our previous joint work [3] with Boris Chorny. We supply model structures and Quillen equivalences underlying Goodwillie's constructions on the homotopy level for functors between simplicial model categories satisfying mild hypotheses.
\end{abstract}

55U35; $18 \mathrm{G} 55$

\section{Introduction}

In a series of three papers $[9 ; 10 ; 11]$, Tom Goodwillie developed a method of analyzing homotopy functors between various categories of topological spaces and spectra, with concrete applications towards Waldhausen's algebraic $K$-theory of spaces. The resulting theory has been extended to other situations (such as categories of chain complexes and Weiss's orthogonal calculus), and successfully applied in many different areas of algebraic and geometric topology, including chromatic stable homotopy theory, nonrealization results for unstable modules over the Steenrod algebra, and computations of unstable homotopy groups of spheres. An overview addressing several of these topics can be found in the article by Kuhn [16].

The basic intuition behind Goodwillie's approach comes from classical calculus, which studies smooth functions via linear or polynomial approximations. Suppose that $F$ is an endofunctor on the category of pointed topological spaces preserving weak homotopy equivalences, such as $\Omega \Sigma$. A priori, the homotopy groups of values of $F$ are not computable. However, suppose that $F$ is excisive in the sense that $F$ sends homotopy pushout squares (such as a Mayer-Vietoris square) to homotopy pullback squares (leading to long exact sequences of homotopy groups). Then the homotopy groups of values of $F$ form a homology theory. Homology theories are in principle computable for many spaces, and can be studied via their representing spectra. Goodwillie associates to a homotopy functor $F$ a tower of endofunctors

$$
F \rightarrow \cdots \rightarrow P_{n+1} F \rightarrow P_{n} F \rightarrow \cdots \rightarrow P_{1} F \rightarrow P_{0} F
$$


in which the functor $P_{n} F$ satisfies an $n^{\text {th }}$ order excision and the map $F \rightarrow P_{n} F$ is initial up to weak equivalence among all maps from $F$ with $n$-excisive target. For instance, the functor $P_{0} F$ is weakly equivalent to the constant functor at $F(*)$, the functor $P_{1} F$ is "the best" excisive approximation to $F$, and the homotopy groups of the homotopy fiber of $P_{1} F \rightarrow P_{0} F$ are a reduced homology theory, represented by a spectrum $\partial^{1} F$. More generally (and very surprisingly) Goodwillie determines the "homogeneous" fibers of this tower in the sense that, for any $n \geq 1$, there is a spectrum $\partial^{n} F$ with $\Sigma_{n}$-action and a natural weak equivalence

$$
D_{n} F(X)=\operatorname{hofib}\left(P_{n} F(X) \rightarrow P_{n-1} F(X)\right) \simeq \Omega^{\infty}\left(\partial^{n} F \wedge X^{\wedge n}\right)_{h \Sigma_{n}},
$$

where $\Sigma_{n}$ acts by permutation on $X^{\wedge n}$. In other words, the layers of the Goodwillie tower (1-1) are governed by spectra, whence the associated spectral sequence computes unstable homotopy groups from stable input. We cannot resist to point out the similarity with the formula for the $n^{\text {th }}$ homogeneous term

$$
\frac{f^{(n)}(0) x^{n}}{n !}
$$

in the Taylor series of a smooth function $f$ around zero. Indeed, Goodwillie calls the $\Sigma_{n}$-spectrum $\partial^{n} F$ the $n^{\text {th }}$ derivative of $F$ at $*$, and produces a corresponding derivative at any pointed topological space. Goodwillie's third and foundational paper [11] contains this classification of homogeneous functors, which is basically subsumed by a commutative diagram of equivalences of homotopy categories:

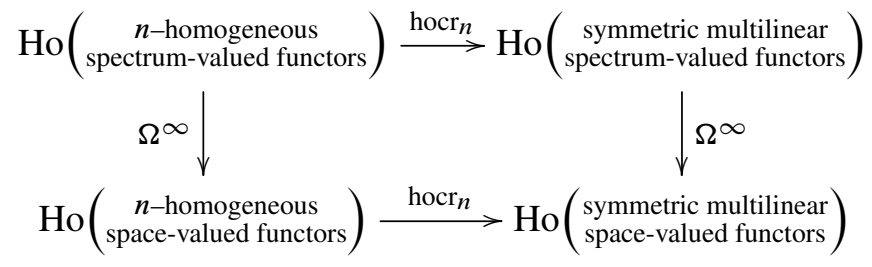

The right-hand side of this diagram consists of symmetric functors in $n$ variables which are linear in each variable; on the left-hand side are $n$-homogeneous functors. The horizontal functor is the $n^{\text {th }}$ homotopy cross effect, which Goodwillie simply denotes $\mathrm{cr}_{n}$. The classification concludes via evaluation on the $n$-tuple $\left(S^{0}, \ldots, S^{0}\right)$ of zero spheres, which constitutes an equivalence from the homotopy category of symmetric multilinear spectrum-valued homotopy functors to the stable homotopy category of spectra with $\Sigma_{n}$-action. Whereas Goodwillie's original proofs of the basic theorems usually involve certain connectivity assumptions, his more recent proofs in [11] consist of clever diagram manipulations which apply in great generality (see also Rezk [20]). In fact, this generality is one reason "for reworking this whole theory in the context of 
closed model categories" [11, page 655], which is the present goal. This goal has been addressed already by Kuhn [16], Biedermann, Chorny and Röndigs [3], Lurie [17], Barnes and Oman [1] (for orthogonal calculus), and most recently by Pereira [19].

Our "reworking" obtained in this article provides not only a lift of the above classification from the level of homotopy categories to the level of model categories, but also a generalization of Goodwillie's calculus to more general model categories. In order to describe this more precisely, let $\mathcal{S}$ be the category of pointed simplicial sets. Given a pointed simplicial model category $\mathcal{D}$, let $\operatorname{Sp}(\mathcal{D})$ denote the stable model category of spectra in $\mathcal{D}$. Finally, let $\mathcal{F}$ be the category of pointed simplicial functors from $\mathcal{C}$ to $\mathcal{D}$. Here $\mathcal{C}$ is a small full subcategory of a pointed simplicial model category $\mathcal{B}$, and both $\mathcal{D}$ and $\mathcal{B}$ are required to satisfy further, but not too restrictive, conditions, which are given in Conventions 3.8, 4.7, 5.2, and 6.1. We explicitly describe a sequence

$$
\mathcal{F}_{\mathrm{hf}} \rightarrow \cdots \rightarrow \mathcal{F}_{(n+1) \text {-exc }} \rightarrow \mathcal{F}_{n-\mathrm{exc}} \rightarrow \cdots \rightarrow \mathcal{F}_{1-\mathrm{exc}} \rightarrow \mathcal{F}_{0-\mathrm{exc}} \simeq *
$$

of left Bousfield localizations of a homotopy functor model structure on $\mathcal{F}$, such that the respective fibrant replacements are $n$-excisive approximations. This lifts Goodwillie's tower (1-1). Furthermore, right Bousfield localization supplies "fiber sequences"

$$
\mathcal{F}_{n \text {-hom }} \rightarrow \mathcal{F}_{n-\text { exc }} \rightarrow \mathcal{F}_{(n-1) \text {-exc }}
$$

of model structures for every $n$, such that cofibrant replacement in $\mathcal{F}_{n \text {-hom }}$ yields an $n$-reduced approximation. In order to complete the description of the layers of the tower, we supply model structures that promotes Goodwillie's diagram (1-3) to a commuting diagram of Quillen equivalences. In the special case where $\mathcal{C}$ is the category of finite pointed simplicial sets, the model structure for symmetric multilinear functors is shown to be Quillen equivalent to the stable model category of spectra in $\mathcal{D}$ with a $\Sigma_{n}$-action, thus also lifting Goodwillie's derivative to the level of model categories.

This article applies to the target $\mathcal{D}=\mathcal{S}$, the category of all pointed simplicial sets, with the full subcategory of finite pointed simplicial sets $\mathcal{C}=\mathcal{S}^{\text {fin }}$ as source. Already in this case, our results extend those in our paper with Chorny [3], which constructed homogeneous model structures only for spectrum-valued functors. Moreover, we now cover the important variation given by the relative setting, where one considers simplicial sets retractive over a fixed simplicial set $K$. Other possible applications, to be investigated elsewhere, are equivariant homotopy theory, and homotopy theory of simplicial sheaves with respect to suitable Grothendieck topologies.

The category of finite pointed CW complexes does not satisfy the conditions we impose on the source category for the construction of the homotopy functor model structure. 
However, all finite $\mathrm{CW}$ complexes are both fibrant and cofibrant and, thus, all simplicial functors defined on finite CW complexes are automatically homotopy functors. In this case, the homotopy functor model structure is not needed and one can perform all the constructions in this article by using the projective model structure or the cross effect model structure directly. Shortly, finite pointed $\mathrm{CW}$ complexes as source category $\mathcal{C}$ and all pointed topological spaces as target category $\mathcal{D}$ qualify as further examples.

Jacob Lurie describes in [17], among many other things, the $n$-excisive part and derivatives in terms of $\infty$-categories for functors between certain $\infty$-categories. Our model structures provide an alternative approach, which should be useful for the practitioners of "calculus", since it upgrades Goodwillie's results on spaces and their generalizations obtained by Pereira [19] from the level of homotopy categories to the level of model categories.

As a guideline to the reader, we offer a short summary of the contents of this article, followed by diagrams indicating the various model structures.

Section 2 supplies the necessary prerequisites on enriched category theory, in particular for functors of several variables, and discusses differences between the unpointed simplicial and the pointed simplicial case. Theorem 2.14 provides projective model structures on functor categories as a starting ground. Section 3 introduces symmetric functors and the categorical cross effect. Theorem 3.19 supplies the cross effect model structure, a modification of the projective model structure with more cofibrations, such that the cross effect becomes a right Quillen functor, with Goodwillie's homotopy cross effect as its right derived functor (Proposition 3.25). Section 4 develops homotopy functor model structures based on mild hypotheses (Convention 4.7), refining and extending the results of [7]. Both the projective and the cross effect model structure may serve as a basis, as explained in Theorem 4.14. Section 5 contains the description of the Goodwillie tower (1-1) on the level of model categories in the following sense: Theorem 5.8 supplies, for every natural number $n$, an $n$-excisive model structure as a suitable left Bousfield localization of the homotopy functor model structures given in Section 4. Furthermore, multilinear model structures are introduced in Theorem 5.20, in order to model Goodwillie's classification of homogeneous functors. Theorem 5.27 shows that, up to Quillen equivalence, symmetric multilinear functors on finite pointed simplicial sets are just spectra with a symmetric group action. Section 6 introduces homogeneous model structures in Theorem 6.4 and completes the classification of homogeneous functors via diagram (6-2). With Lemma 6.24 we supply a characterization of homogeneous cofibrations.

The plethora of model structures on $\mathcal{F}=\operatorname{Fun}(\mathcal{C}, \mathcal{D})$ can be organized into the following schematic diagram of left Quillen identity functors, where an arrow pointing to the 
right represents left Bousfield localization (keep cofibrations, add weak equivalences), a downward arrow displays a special Quillen equivalence (add cofibrations, keep equivalences) and the single upward arrow is a right Bousfield localization (add equivalences, keep fibrations):

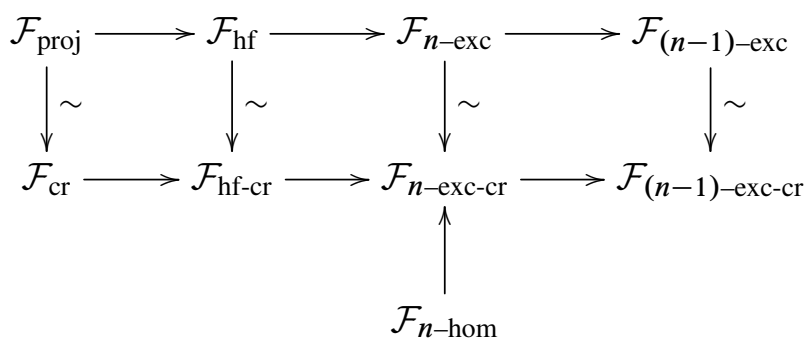

Notation 1.1 The category of simplicial sets (unpointed spaces) is denoted $\mathcal{U}$, and the category of pointed simplicial sets (spaces) is denoted $\mathcal{S}$. The corresponding full subcategories of finite (pointed) simplicial sets are denoted $\mathcal{U}^{\text {fin }}$ and $\mathcal{S}^{\text {fin }}$, respectively. Left adjoints are always on top or to the left. A terminal object in a category $\mathcal{C}$ will be denoted $*_{C}$ or simply $*$.

Acknowledgements We would like to thank Bill Dwyer and André Joyal for many encouraging and enlightening discussions. We thank Luís Alexandre Pereira and the referee for their detailed and helpful comments. The second author was partially supported by DFG grant RO 3867/1-1.

\section{Enriched functors}

This section recalls the necessary prerequisites on enriched category theory, in particular for functors of several variables. Special emphasis is given on the differences between the unpointed simplicial and the pointed simplicial case in Section 2.4.

\subsection{Preliminaries on enriched functors}

References for enriched category theory are Borceux [4], Eilenberg and Kelly [8] and Kelly [15]. This section mainly presents notation.

Convention 2.1 Let $(\mathcal{V}, \otimes, I)$ be a closed symmetric monoidal category.

The two main examples of closed symmetric monoidal categories are mentioned in Notation 1.1: the category $(\mathcal{U}, \times, *)$ of simplicial sets equipped with the categorical product and the category $\left(\mathcal{S}, \wedge, S^{0}\right)$ of pointed simplicial sets equipped with the smash product. 
Notation 2.2 The $\mathcal{V}$-object of morphisms from $A$ to $B$ in any given $\mathcal{V}$-category $\mathcal{C}$ is denoted $\mathcal{V}_{\mathcal{C}}(A, B)$. The category of $\mathcal{V}$-functors from a small $\mathcal{V}$-category $\mathcal{C}$ to another $\mathcal{V}$-category $\mathcal{D}$ is again a $\mathcal{V}$-category, denoted by $\operatorname{Fun}_{\mathcal{V}}(\mathcal{C}, \mathcal{D})$ or simply $\operatorname{Fun}(\mathcal{C}, \mathcal{D})=\mathcal{D}^{\mathcal{C}}$ if no confusion may arise. One example is the functor category $\mathcal{D}^{G}$, where $G$ is a monoid, considered as a (discrete) $\mathcal{V}$-category with a single object.

Definition 2.3 Given $\mathcal{V}$-categories $\mathcal{C}_{i}$ for $i=1, \ldots, n$, the monoidal product category $\mathcal{C}_{1} \otimes \cdots \otimes \mathcal{C}_{n}$ has as objects ordered $n$-tuples $\left(K_{1}, \ldots, K_{n}\right)$ of objects $K_{i}$ in $\mathcal{C}_{i}$, and as $\mathcal{V}$-object of morphisms from $\underline{K}=\left(K_{1}, \ldots, K_{n}\right)$ to $\underline{L}=\left(L_{1}, \ldots, L_{n}\right)$ the $n$-fold monoidal product

$$
\mathcal{V}_{\mathcal{C}_{1} \otimes \cdots \otimes \mathcal{C}_{n}}(\underline{K}, \underline{L}):=\bigotimes_{i=1}^{n} \mathcal{V}_{\mathcal{C}}\left(K_{i}, L_{i}\right)
$$

Composition and units are readily introduced, giving $\mathcal{C}_{1} \otimes \cdots \otimes \mathcal{C}_{n}$ a $\mathcal{V}$-category structure.

Of course it suffices to give Definition 2.3 for two factors. The general case is presented in view of discussing enriched functors in several variables.

Example 2.4 In the case where the closed symmetric monoidal base category is $(\mathcal{U}, \times, *)$, the underlying category of a monoidal product category coincides with the ordinary product category; an observation which could be abbreviated as $\mathcal{C} \otimes \mathcal{U} D \cong \mathcal{C} \times \mathcal{D}$. This is different in the case of $\left(\mathcal{S}, \wedge, S^{0}\right)$. For example, any object of the form $(K, *)$ or $(*, L)$ in $\mathcal{S} \wedge \mathcal{S}$ is a zero object.

Definition 2.5 Let $\mathcal{C}$ and $\mathcal{D}$ be $\mathcal{V}$-categories, with $\mathcal{C}$ small. Recall that if $\mathcal{D}$ is tensored over $\mathcal{V}$, there is a $\mathcal{V}$-functor

$$
\operatorname{Fun}(\mathcal{C}, \mathcal{V}) \otimes \mathcal{D} \rightarrow \operatorname{Fun}(\mathcal{C}, \mathcal{D})
$$

sending $(D, \underset{\sim}{X})$ to the $\mathcal{V}$-functor

$$
\underset{\sim}{X} \otimes D: C \mapsto \underset{\sim}{X}(C) \otimes D .
$$

For fixed $D$ in $\mathcal{D}$ the $\mathcal{V}$-functor $\underset{\sim}{X} \mapsto \underset{\sim}{X} \otimes D$ has a right adjoint $\underset{\sim}{Y} \mapsto{\underset{\sim}{Y}}^{D}$, where $\left({\underset{\sim}{Y}}^{D}\right)(C)=\mathcal{V}_{\mathcal{D}}(D, \underset{\sim}{Y}(C))$. For fixed $\underset{\sim}{X} \in \operatorname{Fun}(\mathcal{C}, \mathcal{V})$ the functor $\widetilde{\sim} \mapsto \underset{\sim}{X} \otimes D$ has a right adjoint

$$
\underset{\sim}{Y} \mapsto \operatorname{hom}(\underset{\sim}{X}, \underset{\sim}{Y})=\int_{C}(\underset{\sim}{Y}(C))_{\sim}^{X(C)} \in \mathcal{D}
$$

if $\mathcal{D}$ is also cotensored over $\mathcal{V}$. Slightly adapting these definitions supplies $\mathcal{V}$-functors

$$
\operatorname{Fun}(\mathcal{C}, \mathcal{D}) \otimes \mathcal{V} \rightarrow \operatorname{Fun}(\mathcal{C}, \mathcal{D})
$$


and

$$
\mathcal{V}^{\text {op }} \otimes \operatorname{Fun}(\mathcal{C}, \mathcal{D}) \rightarrow \operatorname{Fun}(\mathcal{C}, \mathcal{D})
$$

giving the functor category $\operatorname{Fun}(\mathcal{C}, \mathcal{D})$ the usual structure of a tensored and cotensored $\mathcal{V}$-category.

Notation 2.6 The covariant $\mathcal{V}$-functor represented by the object $C \in \mathcal{C}$ is denoted

$$
R^{C}=R_{\mathcal{C}}^{C}=\mathcal{V}_{\mathcal{C}}\left(C,{ }_{-}\right): \mathcal{C} \rightarrow \mathcal{V}
$$

Lemma 2.7 (Yoneda) Let $C$ be an object in $\mathcal{C}$ and $\underset{\sim}{Y}$ in $\operatorname{Fun}(\mathcal{C}, \mathcal{D})$. The $\mathcal{V}$-natural transformation

$$
\left\{\underset{\sim}{Y}(C) \rightarrow \underset{\sim}{Y}(D)^{R^{C}(D)}\right\}_{D \in \mathcal{C}}
$$

induces an isomorphism

$$
\underset{\sim}{Y}(C) \cong \operatorname{hom}\left(R^{C}, \underset{\sim}{Y}\right) .
$$

Definition 2.8 Let $\mathcal{C}$ be a small $\mathcal{V}$-category. The objectwise tensor product of $\underset{\sim}{X}$ and $\underset{\sim}{Y}$ in $\operatorname{Fun}(\mathcal{C}, \mathcal{V})$, denoted by $\underset{\sim}{X} \otimes \underset{\sim}{Y}$, is given by the equation

$$
(\underset{\sim}{X} \otimes \underset{\sim}{Y})(C):=\underset{\sim}{X}(C) \otimes \underset{\sim}{Y}(C) \text {. }
$$

\subsection{The projective model structure}

For terminology concerning model categories, consider Hirschhorn [12] or Hovey [13]. A (co)fibration which is also a weak equivalence will be called an acyclic (co)fibration.

Convention 2.9 Let $\mathcal{V}$ be a symmetric monoidal model category.

Again the main examples are (pointed) simplicial sets, with monomorphisms as cofibrations and maps inducing homotopy equivalences after geometric realization as weak equivalences. As explained in the references mentioned, a $\mathcal{V}$-model category is tensored and cotensored over $\mathcal{V}$, and the compatibility of the model structures with the enrichment is expressed using the following definition.

Definition 2.10 Let $f: A \rightarrow B$ and $g: C \rightarrow D$ be two maps in $\mathcal{V}$. The map

$$
f \square g:(A \otimes D) \cup_{(A \otimes C)} B \otimes C \rightarrow B \otimes D
$$

induced by $f \otimes D$ and $B \otimes g$ is called the pushout product of $f$ and $g$. The analogous construction where $g$ is a map in a tensored $\mathcal{V}$-category $\mathcal{D}$ with pushouts yields a map $f \square g$ is a map in $\mathcal{D}$. 
Hirschhorn calls it the pushout corner map in [12, 9.3.5.(2)]. In order to equip the category $\operatorname{Fun}(\mathcal{C}, \mathcal{D})$ of $\mathcal{V}$-functors from a small $\mathcal{V}$-category $\mathcal{C}$ to a $\mathcal{V}$-model category $\mathcal{D}$ with the projective model structure (whose weak equivalences and fibrations are defined objectwise), certain assumptions on $\mathcal{D}$ are necessary.

Definition 2.11 Let $\mathcal{V}$ be a monoidal model category and let $\mathcal{D}$ be a $\mathcal{V}$-model category. The $\mathcal{V}$-model category $\mathcal{D}$ satisfies the $\mathcal{V}$-monoid axiom if the following property holds: Let acof ${ }_{\mathcal{D}}$ be the class of acyclic cofibrations in $\mathcal{D}$. Let $\mathcal{E}_{\mathcal{D}}$ be the class of relative cell complexes in $\mathcal{D}$ generated by the class of morphisms

$$
\left\{j \otimes A \mid j \in \operatorname{acof}_{\mathcal{D}}, A \in \mathrm{Ob} \mathcal{V}\right\} .
$$

Then every morphism in $\mathcal{E}_{\mathcal{D}}$ is a weak equivalence.

Definition 2.12 Let $\mathcal{V}$ be a monoidal model category, and let $\mathcal{D}$ be a $\mathcal{V}$-model category. Let $\mathcal{F}_{\mathcal{D}}$ be the class of relative cell complexes in $\mathcal{D}$ generated by the class of morphisms

$$
\left\{i \otimes A \mid i \in \operatorname{cof}_{\mathcal{D}}, A \in \mathrm{Ob} \mathcal{V}\right\} .
$$

The $\mathcal{V}$-model category $\mathcal{D}$ is $\mathcal{V}$-left proper if weak equivalences in $\mathcal{D}$ are closed under cobase change along morphisms in $\mathcal{F}_{\mathcal{D}}$.

Remark 2.13 If all objects in $\mathcal{V}$ are cofibrant, the $\mathcal{V}$-monoid axiom holds automatically in any $\mathcal{V}$-model category $\mathcal{D}$. Furthermore, in that case, $\mathcal{V}$-left properness is equivalent to left properness. This holds in particular for the cases $\mathcal{V}=\mathcal{S}$ or $\mathcal{U}$.

Theorem 2.14 Let $\mathcal{D}$ be a bicomplete $\mathcal{V}$-model category which is cofibrantly generated. If the $\mathcal{V}$-monoid axiom holds in $\mathcal{D}$, the category

$$
\operatorname{Fun}_{\mathcal{V}}(\mathcal{C}, \mathcal{D})
$$

of $\mathcal{V}$-functors from a small $\mathcal{V}$-category $\mathcal{C}$ to $\mathcal{D}$ carries a cofibrantly generated model structure, where the weak equivalences and fibrations are defined objectwise. If the model structure on $\mathcal{D}$ is right proper, so is the projective model structure. If the model structure on $\mathcal{D}$ is $\mathcal{V}$-left proper, the projective model structure is left proper.

Proof This follows by adapting the proof of Dundas, Röndigs and Østvær [7, Theorem 4.4]. Left properness is shown as in the proof of [7, Corollary 4.8]. Following standard terminology, this model structure will be referred to as the projective model structure. For future reference, generating sets for cofibrations and acyclic cofibrations 
in the projective model structure are constructed as follows: Tensoring the functor $R^{K}$ with an object $E \in \operatorname{Ob}(\mathcal{D})$ yields a $\mathcal{V}$-functor

$$
R^{K} \otimes E: \mathcal{C} \rightarrow \mathcal{D}, \quad L \mapsto \mathcal{V}_{\mathcal{C}}(K, L) \otimes E .
$$

The $\mathcal{V}$-Yoneda Lemma 2.7 implies that any $\mathcal{V}$-functor $\underset{\sim}{X}: \mathcal{C} \rightarrow \mathcal{D}$ is naturally isomorphic to the coend

$$
\int_{K \in \mathcal{C}} R^{K} \otimes \underset{\sim}{X}(K)
$$

Given generating sets $I_{\mathcal{D}}$ and $J_{\mathcal{D}}$ for the model structure on $\mathcal{D}$, the sets

$$
\begin{aligned}
& I_{\text {Fun } \mathcal{V}(\mathcal{C}, \mathcal{D})}^{\text {proj }}:=\left\{R^{K} \otimes i \mid K \in \mathrm{Ob}(\mathcal{C}), i \in I_{\mathcal{D}}\right\} \\
& J_{\text {Fun }_{\mathcal{V}}(\mathcal{C}, \mathcal{D})}^{\text {proj }}:=\left\{R^{K} \otimes j \mid K \in \mathrm{Ob}(\mathcal{C}), j \in J_{\mathcal{D}}\right\}
\end{aligned}
$$

are generating (acyclic) cofibrations for the projective model structure.

\subsection{Enriched functors in several variables}

A $\mathcal{V}$-functor in several variables is simply a $\mathcal{V}$-functor

$$
\mathcal{C}_{1} \otimes \cdots \otimes \mathcal{C}_{n} \rightarrow \mathcal{D}
$$

where $\mathcal{D}$ and $\mathcal{C}_{i}$ for $i=1, \ldots, n$ are $\mathcal{V}$-categories. In order to translate between $\mathcal{V}$-functors in several variables and in a single variable, let $\mathcal{I}_{\mathcal{V}}$ denote the $\mathcal{V}$-category given by the full subcategory of $\mathcal{V}$ containing as its single object the unit $I$. In other words, it is a $\mathcal{V}$-category with one object, also denoted $I$, and endomorphism object $I$. For every $\mathcal{V}$-category $\mathcal{A}$ there are canonical unit isomorphisms

$$
\mathcal{I}_{\mathcal{V}} \otimes \mathcal{A} \stackrel{\mathcal{A}}{\cong} \mathcal{A} \otimes \mathcal{I}_{\mathcal{V}}
$$

of $\mathcal{V}$-categories.

For any object $B$ in a $\mathcal{V}$-category $\mathcal{B}$, there is a $\mathcal{V}$-functor

$$
i_{B}: \mathcal{A} \otimes \mathcal{I}_{\mathcal{V}} \rightarrow \mathcal{A} \otimes \mathcal{B}
$$

which is given on objects by $(A, I) \mapsto(A, B)$ and on morphisms by

$$
\mathcal{V}_{\mathcal{A}}\left(A_{1}, A_{2}\right) \otimes I \rightarrow \mathcal{V}_{\mathcal{A}}\left(A_{1}, A_{2}\right) \otimes \mathcal{V}_{\mathcal{B}}(B, B),
$$

where $I \rightarrow \mathcal{V}_{\mathcal{B}}(B, B)$ is the canonical unit map. Of course, there is an analogous functor $i_{A}: \mathcal{I}_{\mathcal{V}} \otimes \mathcal{B} \rightarrow \mathcal{A} \otimes \mathcal{B}$ for every $A$ in $\mathcal{A}$. Given a $\mathcal{V}$-functor $G: \mathcal{A} \otimes \mathcal{B} \rightarrow \mathcal{D}$, every object $B$ in $\mathcal{B}$ defines the partial functor $G_{B}: \mathcal{A} \rightarrow \mathcal{D}$ by composition:

$$
\mathcal{A} \stackrel{\gamma_{\mathcal{A}}}{\cong} \mathcal{A} \otimes \mathcal{I}_{\mathcal{V}} \stackrel{i_{B}}{\longrightarrow} \mathcal{A} \otimes \mathcal{B} \stackrel{G}{\longrightarrow} \mathcal{D}
$$


The functor $G$ is uniquely determined by all its partial functors $G_{A}$ and $G_{B}$ :

Proposition 2.15 (Eilenberg and Kelly [8, Proposition 4.2]) Suppose that, for all objects $A$ of $\mathcal{A}$ and $B$ of $\mathcal{B}$, there are $\mathcal{V}$-functors $G_{A}: \mathcal{B} \rightarrow \mathcal{D}$ and $G_{B}: \mathcal{A} \rightarrow \mathcal{D}$ with the property $G_{A}(B)=G_{B}(A)=: G(A, B)$. Then there exists a unique $\mathcal{V}$-functor $G: \mathcal{A} \otimes \mathcal{B} \rightarrow \mathcal{D}$ with $\left\{G_{A}\right\}$ and $\left\{G_{B}\right\}$ as partial functors if and only if the following diagram commutes:

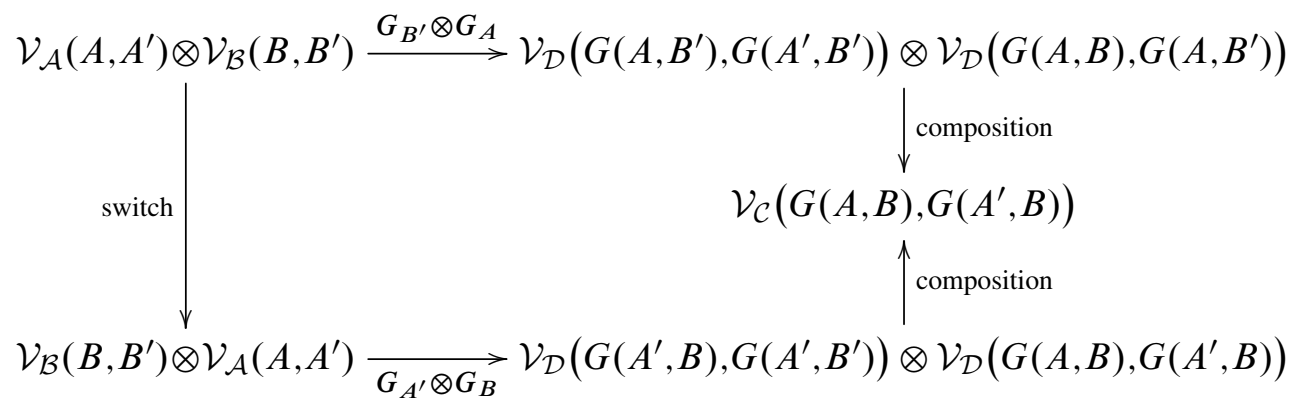

In other words, a $\mathcal{V}$-functor from a monoidal product category is essentially a functor in $n$ variables which is componentwise enriched over $\mathcal{V}$. The analogous result for $\mathcal{V}$-natural transformations will be used as well.

Proposition 2.16 (Eilenberg and Kelly [8, Proposition 4.12]) Let $\mathcal{V}$ be a symmetric monoidal category and $T, S: \mathcal{A} \otimes \mathcal{B} \rightarrow \mathcal{D}$ be two $\mathcal{V}$-functors. For all objects $A$ in $\mathcal{A}$ and $B$ in $\mathcal{B}$ let

$$
\alpha_{A, B}: S(A, B) \rightarrow T(A, B)
$$

be a map in the underlying category of $\mathcal{D}$. The maps $\alpha_{A, B}$ are the components of a $\mathcal{V}$-natural transformation $\alpha: S \rightarrow T$ if and only if, for each $A$, the map $\alpha_{A, B}$ is the $B$-component of a $\mathcal{V}$-natural transformation $\alpha_{A}: S_{A} \rightarrow T_{A}$ and, for each $B$, the map $\alpha_{A, B}$ is the $A$-component of a $\mathcal{V}$-natural transformation $\alpha_{B}: S_{B} \rightarrow T_{B}$.

Recall that a terminal object in a category $\mathcal{C}$ is denoted $*_{\mathcal{C}}$ or simply $*$.

Definition 2.17 Suppose that the categories $\mathcal{C}$ and $\mathcal{D}$ admit a terminal object. A functor $F: \mathcal{C} \rightarrow \mathcal{D}$ is called reduced if $F\left(*_{\mathcal{C}}\right) \cong *_{\mathcal{D}}$. A functor $F$ to $\mathcal{D}$ in $n$ variables is called multireduced if

$$
F\left(K_{1}, \ldots, K_{n}\right) \cong *_{\mathcal{D}}
$$

whenever $K_{i}$ is a terminal object for at least one $i \in\{1, \ldots, n\}$. 
Remark 2.18 Suppose that $\mathcal{V}$ and the categories $\mathcal{C}_{i}$ are pointed categories. Then every representable $\mathcal{V}$-functor $\mathcal{C}_{1} \otimes \cdots \otimes \mathcal{C}_{n} \rightarrow \mathcal{V}$ is multireduced. Hence if $\mathcal{D}$ is a cocomplete $\mathcal{V}$-category, every object in $\operatorname{Fun}\left(\mathcal{C}_{1} \otimes \cdots \otimes \mathcal{C}_{n}, \mathcal{D}\right)$ is multireduced as a colimit of representable functors by the $\mathcal{V}$-Yoneda Lemma 2.7 .

\subsection{Smash product and product categories}

This section discusses monoidal product categories in the special cases of unpointed and pointed simplicial sets. Since the functor $u: \mathcal{S} \rightarrow \mathcal{U}$ forgetting the base point is lax symmetric monoidal, every $\mathcal{S}$-category $\mathcal{C}$ has an associated $\mathcal{U}$-category $u \mathcal{C}$ by simply forgetting base points in all morphism objects.

Lemma 2.19 Let $\mathcal{C}$ and $\mathcal{D}$ be $\mathcal{S}$-categories. Then $u \mathcal{C} \times u \mathcal{D}$ is an $\mathcal{S}$-category in a natural way.

Proof Given objects $\underline{K}=\left(K_{1}, K_{2}\right), \underline{L}=\left(L_{1}, L_{2}\right), \underline{M}=\left(M_{1}, M_{2}\right) \in u \mathcal{C} \times u \mathcal{D}$, the simplicial set

$$
\mathcal{U}_{u \mathcal{C} \times u \mathcal{D}}\left(\left(K_{1}, K_{2}\right),\left(L_{1}, L_{2}\right)\right)=u \mathcal{S}_{\mathcal{C}}\left(K_{1}, L_{1}\right) \times u \mathcal{S}_{\mathcal{D}}\left(K_{2}, L_{2}\right)
$$

is naturally a pointed simplicial set. The $\mathcal{S}$-composition is induced by the $\mathcal{U}$-composition map

$$
\mathcal{S}(\underline{L}, \underline{M}) \times \mathcal{S}(\underline{K}, \underline{L}) \rightarrow \mathcal{S}(\underline{K}, \underline{L})
$$

since if $\underline{f}=*$ or $\underline{g}=*$, then $f_{i} \circ g_{i}=*$ for all $1 \leq i \leq n$. The unit

$$
S^{0} \rightarrow u \mathcal{S}_{u \mathcal{C} \times u \mathcal{D}}(\underline{K}, \underline{K})
$$

is induced by the diagonal. Associativity and unitality of the $\mathcal{U}$-composition imply associativity and unitality for the $\mathcal{S}$-composition.

Notation 2.20 Let $\mathcal{C}$ and $\mathcal{D}$ be $\mathcal{S}$-categories. The $\mathcal{S}$-category from Lemma 2.19 is denoted $\mathcal{C} \times \mathcal{D}$.

Lemma 2.21 Let $\mathcal{C}$ and $\mathcal{D}$ be $\mathcal{S}$-model categories. Then $\mathcal{C} \times \mathcal{D}$ is an $\mathcal{S}$-model category with the componentwise model structure.

Proof The category underlying the $\mathcal{S}$-category $\mathcal{C} \times \mathcal{D}$ is simply the product category. Hence, the existence of the componentwise model structure follows from Hovey [13, Example 1.1.6]. It remains to prove that $\mathcal{C} \times \mathcal{D}$ is tensored and cotensored over $\mathcal{S}$, and to verify the pushout product axiom. Tensor and cotensor are defined componentwise. It is straightforward to check that they constitute $\mathcal{S}$-functors which are part of $\mathcal{S}$ adjunctions. The pushout product axiom follows immediately. 
Definition 2.22 Let $\mathcal{C}_{1}, \ldots, \mathcal{C}_{n}$ be $\mathcal{S}$-categories. The canonical functor

$$
p: \mathcal{C}_{1} \times \cdots \times \mathcal{C}_{n} \rightarrow \mathcal{C}_{1} \wedge \cdots \wedge \mathcal{C}_{n}
$$

being the identity on objects and the quotient map from the Cartesian product to the smash product on morphisms is an $\mathcal{S}$-functor. If each $\mathcal{C}_{i}$ is small and $\mathcal{D}$ is another $\mathcal{S}$-category, $p$ induces an $\mathcal{S}$-adjoint pair

$$
p_{*}: \operatorname{Fun}\left(\mathcal{C}_{1} \times \cdots \times \mathcal{C}_{n}, \mathcal{D}\right) \rightleftarrows \operatorname{Fun}\left(\mathcal{C}_{1} \wedge \cdots \wedge \mathcal{C}_{n}, \mathcal{D}\right): p^{*}
$$

Lemma 2.23 Let $\mathcal{D}$ be an $\mathcal{S}$-model category. The adjoint pair $\left(p_{*}, p^{*}\right)$ is a Quillen pair of projective model structures. The functor $p^{*}$ preserves and detects objectwise weak equivalences and objectwise fibrations.

Proof This is immediate, since $p^{*}$ is precomposition with a functor being the identity on objects.

For the sake of brevity, notational differences between an $\mathcal{S}$-category and its associated $\mathcal{U}$-category may be ignored in the following discussion. We denote by $\{*\}=\mathcal{I}_{\mathcal{U}}$ the unpointed simplicial category with one object and no non-identity morphisms and by $\left\{S^{0}\right\}=\mathcal{I}_{\mathcal{S}}$ the corresponding $\mathcal{S}$-category. There is exactly one $\mathcal{U}$-functor

$$
N:\{*\} \rightarrow\left\{S^{0}\right\}
$$

and it is given on underlying simplicial sets by sending $*$ to the non-basepoint. Let $\mathcal{A}$ and $\mathcal{B}$ be $\mathcal{S}$-categories (not necessarily containing a zero object). There is a canonical isomorphism of unpointed simplicial categories

$$
\pi_{\mathcal{A}}: \mathcal{A} \stackrel{\cong}{\longrightarrow} \mathcal{A} \times\{*\}
$$

and an analogous one with entries switched. The functors $N$ and $\pi_{\mathcal{A}}$ are unpointed simplicial but not pointed simplicial. In particular, the functor

$$
J: \mathcal{A} \times\{*\} \stackrel{(\mathrm{id}, N)}{\longrightarrow} \mathcal{A} \times\left\{S^{0}\right\} \stackrel{p}{\longrightarrow} \mathcal{A} \wedge\left\{S^{0}\right\}
$$

is unpointed simplicial. For any $B$ in $\mathcal{B}$ we obtain an unpointed simplicial functor

$$
i_{B}: \mathcal{A} \times\{*\} \rightarrow \mathcal{A} \times \mathcal{B}
$$

given on objects by $(A, *) \mapsto(A, B)$ and on morphisms by $(f, *) \rightarrow\left(f, \mathrm{id}_{B}\right)$. Again, there are also functors $i_{A}$. We hope no confusion with the analogous definition in the pointed case will arise from the indiscriminate notation. 
For every object $B$ in $\mathcal{B}$ the diagram

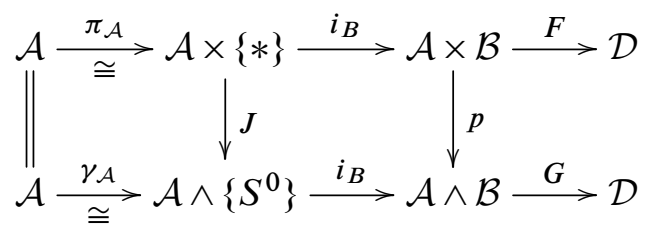

commutes, where the upper row consists of $\mathcal{U}$-functors and the lower row consists of $\mathcal{S}$-functors. Obviously, there is an analogous commutative diagram for every object $A$ in $\mathcal{A}$. Given a $\mathcal{U}$-functor $F$ and an object $B$ as above we define the partial functor $F_{B}$ by composing the upper row. Given an $\mathcal{S}$-functor $G$ and $B$ as above we define the partial functor $G_{B}$ by composing the lower row. Similarly, we define partial functors $F_{A}$ and $G_{A}$ for every $A$ in $\mathcal{A}$. The functor $F$ in (2-2) is $\mathcal{S}$-enriched if and only if $F\left(*_{\mathcal{A}}, *_{\mathcal{B}}\right) \cong *_{\mathcal{D}}$. The latter does not imply that $F$ is multireduced.

Lemma 2.24 Let $\mathcal{A}, \mathcal{B}$ and $\mathcal{D}$ be $\mathcal{S}$-categories. For a $\mathcal{U}$-functor $F: \mathcal{A} \times \mathcal{B} \rightarrow \mathcal{D}$, the following are equivalent:

(1) The functor $F$ is multireduced.

(2) All partial functors of $F$ are reduced.

(3) The functor $F$ is isomorphic to $p^{*} G$ for some $\mathcal{S}$-functor $G: \mathcal{A} \wedge \mathcal{B} \rightarrow \mathcal{D}$.

If these conditions hold, $F$ is in particular an $\mathcal{S}$-functor.

Proof Since all $\mathcal{S}$-functors $G: \mathcal{A} \wedge \mathcal{B} \rightarrow \mathcal{D}$ are multireduced and $p^{*}$ is the identity on objects, (3) implies (1). Obviously, (1) implies (2). Now, assume (2). Then, for all objects $A$ in $\mathcal{A}$ and $B$ in $\mathcal{B}$ the partial functors $F_{A}: \mathcal{B} \rightarrow \mathcal{D}$ and $F_{B}: \mathcal{A} \rightarrow \mathcal{D}$ are $\mathcal{S}-$ functors. The partial functors $G_{A}:=F_{A}$ and $G_{B}:=F_{B}$ assemble by Proposition 2.15 to an $\mathcal{S}$-functor $G: \mathcal{A} \wedge \mathcal{B} \rightarrow \mathcal{D}$. Because the diagram (2-2) commutes, there are canonical isomorphisms

$$
\left(p^{*} G\right)_{A} \cong G_{A}=F_{A} \quad \text { and } \quad\left(p^{*} G\right)_{B} \cong G_{B}=F_{B}
$$

for all $A$ and $B$. This gives (3).

\section{Symmetric functors}

A major tool in Goodwillie's theory is the cross effect, a functorial construction which measures to which extent a given homotopy functor deviates from being excisive. The purpose of this section is to interpret Goodwillie's cross effect construction as a Quillen functor between appropriate model categories. 


\subsection{Symmetric functors}

Definition 3.1 Let $\mathcal{C}$ and $\mathcal{D}$ be $\mathcal{V}$-categories. A $\mathcal{V}$-functor $\underset{\sim}{X}: \mathcal{C} \otimes \cdots \otimes \mathcal{C} \rightarrow \mathcal{D}$ in $n$ variables with values in $\mathcal{D}$ is symmetric if it is equipped with a $\mathcal{V}$-natural isomorphism

$$
\sigma_{\underset{\sim}{X}}: \underset{\sim}{X}\left(K_{1}, \ldots, K_{n}\right) \cong \underset{\sim}{X}\left(K_{\sigma(1)}, \ldots, K_{\sigma(n)}\right)
$$

for every $\sigma \in \Sigma_{n}$, such that the equalities $\operatorname{id}_{\underset{\sim}{X}}=\mathrm{id}$ and $(\tau \sigma)_{\underset{\sim}{X}}=\tau_{\underset{\sim}{X}} \sigma_{\underset{X}{X}}$ hold. A symmetric $\mathcal{V}$-natural transformation is a $\mathcal{V}$-natural transformation between two symmetric functors that respects the symmetry in the obvious way. Let $\operatorname{Fun}_{\text {sym }}\left(\mathcal{C}^{\otimes n}, \mathcal{D}\right)$ denote the corresponding $\mathcal{V}$-category.

The standard example of a symmetric $\mathcal{V}$-functor is the $n$-fold monoidal product

$$
\bigotimes_{i=1}^{n}: \mathcal{V} \otimes \cdots \otimes \mathcal{V} \rightarrow \mathcal{V}
$$

The main example of interest here are cross effect functors, to be described in the next section. In order to introduce model structures for symmetric functors, it will be convenient to describe them as a genuine functor category instead of just a proper subcategory of a functor category.

Convention 3.2 Suppose that the closed symmetric monoidal category $(\mathcal{V}, \otimes, I)$ has finite coproducts, denoted as $\vee$.

Definition 3.3 Let $\mathcal{C}$ be a $\mathcal{V}$-category. The wreath product category $\Sigma_{n} \imath \mathcal{C}^{\otimes n}$ has as its objects $n$-tuples $\left(K_{1}, \ldots, K_{n}\right)$ of objects in $\mathcal{C}$. The morphisms from $\underline{K}=$ $\left(K_{1}, \ldots, K_{n}\right)$ to $\underline{L}=\left(L_{1}, \ldots, L_{n}\right)$ are given by

$$
\mathcal{V}_{\Sigma_{n} \mathcal{C}^{\mathcal{C}} \otimes n}(\underline{K}, \underline{L}):=\bigvee_{\sigma \in \Sigma_{n}} \bigotimes_{i=1}^{n} \mathcal{V}_{\mathcal{C}}\left(K_{i}, L_{\sigma^{-1}(i)}\right)
$$

Composition is defined as it is in the wreath product of groups or, more generally, in a semi-direct product by the formula

(3-1) $\left(\tau,\left(g_{1} \otimes \cdots \otimes g_{n}\right)\right) \circ\left(\sigma,\left(f_{1} \otimes \cdots \otimes f_{n}\right)\right)=\left(\sigma \tau,\left(g_{\sigma^{-1}(1)} f_{1} \otimes \cdots \otimes g_{\sigma^{-1}(n)} f_{n}\right)\right)$.

More formally, composition is a map

$$
\left.\mathcal{V}_{\Sigma_{n} \mathcal{C} \otimes n}(\underline{L}, \underline{M}) \otimes \mathcal{V}_{\Sigma_{n} \mathcal{C} \otimes n}(\underline{K}, \underline{L}) \rightarrow \mathcal{V}_{\Sigma_{n} \mathcal{C} \otimes n}(\underline{K}, \underline{M})\right) .
$$

Observe that the source of the composition map is canonically isomorphic to the term

$$
\bigvee_{(\tau, \sigma) \in \Sigma_{n} \times \Sigma_{n}}\left[\bigotimes_{j=1}^{n} \mathcal{V}_{\mathcal{C}}\left(L_{j}, M_{\tau^{-1}(j)}\right) \otimes \bigotimes_{i=1}^{n} \mathcal{V}_{\mathcal{C}}\left(K_{i}, L_{\sigma^{-1}(i)}\right)\right]
$$


while the target is given by

$$
\bigvee_{\omega \in \Sigma_{n}} \bigotimes_{k=1}^{n} \mathcal{V}_{\mathcal{C}}\left(K_{k}, M_{\omega^{-1}(k)}\right)
$$

Given $\sigma$ and $\tau$, the corresponding summand in the first term is mapped to the summand corresponding to $\omega=\sigma \tau$, with the map being the $n$-fold monoidal product of the $\mathcal{V}$-composition

$$
\mathcal{V}_{\mathcal{C}}\left(L_{\sigma^{-1}(k)}, M_{\tau^{-1}\left(\sigma^{-1}(k)\right)}\right) \otimes \mathcal{V}_{\mathcal{C}}\left(K_{k}, L_{\sigma^{-1}(k)}\right) \rightarrow \mathcal{V}_{\mathcal{C}}\left(K_{k}, M_{\omega^{-1}(k)}\right)
$$

up to a permutation of monoidal factors. This amounts to the formula (3-1). Associativity and identity conditions are checked readily, implying that $\Sigma_{n} \prec \mathcal{C}^{\otimes n}$ is indeed a $\mathcal{V}$-category.

Remark 3.4 Here are two interpretations of this construction.

(1) The $\mathcal{V}$-category $\Sigma_{n} 2 \mathcal{C}^{\otimes n}$ is the $\mathcal{V}$-category of unordered $n$-tuples. More precisely, in $\Sigma_{n} \curlywedge \mathcal{C}^{\otimes n}$ there is for every $\sigma \in \Sigma_{n}$ and every $n$-tuple $\underline{K}$ a canonical map

$$
\underline{K}=\left(K_{1}, \ldots, K_{n}\right) \stackrel{\left(\sigma^{-1}, \mathrm{id} \otimes \cdots \otimes \mathrm{id}\right)}{\longrightarrow}\left(K_{\sigma(1)}, \ldots, K_{\sigma(n)}\right),
$$

that is an isomorphism with inverse $(\sigma, \mathrm{id} \otimes \cdots \otimes \mathrm{id})$. Moreover, any map in $\Sigma_{n} \prec \mathcal{C}^{\otimes n}$ can be written as the composition of such an isomorphism with a map from $\mathcal{C}^{\otimes n}$ :

$$
\begin{aligned}
\left(\sigma, f_{1} \otimes \cdots \otimes f_{n}\right) & =(\sigma, \mathrm{id} \otimes \cdots \otimes \mathrm{id}) \circ\left(\mathrm{id}, f_{1} \otimes \cdots \otimes f_{n}\right) \\
& =\left(\mathrm{id}, f_{\sigma^{-1}(1)} \otimes \cdots \otimes f_{\sigma^{-1}(n)}\right) \circ(\sigma, \mathrm{id} \otimes \cdots \otimes \mathrm{id}) .
\end{aligned}
$$

(2) The $\mathcal{V}$-category $\Sigma_{n} \mathcal{C}^{\otimes n}$ is obtained as the $\mathcal{V}$-Grothendieck construction or $\mathcal{V}$-category of elements of the functor $\Sigma_{n} \rightarrow \mathcal{V}$-Cat sending the unique object to $\mathcal{C}^{\otimes n}$ with the permutation action.

Definition 3.5 For every $\mathcal{V}$-category $\mathcal{C}$ there is a functor

$$
\varepsilon: \mathcal{C}^{\otimes n} \rightarrow \Sigma_{n} \prec \mathcal{C}^{\otimes n}
$$

which is the identity on objects and the inclusion of the summand indexed by the identity in $\Sigma_{n}$ on morphisms: $\left(f_{1} \otimes \cdots \otimes f_{n}\right) \mapsto\left(\right.$ id, $\left.f_{1} \otimes \cdots \otimes f_{n}\right)$.

Lemma 3.6 Let $\mathcal{C}$ and $\mathcal{D}$ be $\mathcal{V}$-categories. Suppose also that $\mathcal{C}$ is small. Precomposition with $\varepsilon$ induces an equivalence

$$
\varepsilon^{*}: \operatorname{Fun}\left(\Sigma_{n} \curlywedge \mathcal{C}^{\otimes n}, \mathcal{D}\right) \rightarrow \operatorname{Fun}_{\text {sym }}\left(\mathcal{C}^{\otimes n}, \mathcal{D}\right)
$$

of $\mathcal{V}$-categories. 
Proof Precomposition with $\varepsilon$ defines a $\mathcal{V}$-functor

$$
\varepsilon^{*}: \operatorname{Fun}\left(\Sigma_{n} \prec \mathcal{C}^{\otimes n}, \mathcal{D}\right) \rightarrow \operatorname{Fun}\left(\mathcal{C}^{\otimes n}, \mathcal{D}\right) .
$$

By construction, every $\mathcal{V}$-functor $\left(\mathcal{V}\right.$-natural transformation) in the image of $\varepsilon^{*}$ is symmetric. The $\mathcal{V}$-functor with target restricted to the category of symmetric functors will also be denoted $\varepsilon^{*}$. Unravelling the definitions shows that a $\mathcal{V}$-functor $\mathcal{C}^{\otimes n} \rightarrow \mathcal{D}$ is symmetric precisely if its domain extends (via the extra data) to the wreath product category $\Sigma_{n} \prec \mathcal{C}^{\otimes n}$, which essentially completes the proof.

Definition 3.7 Let $\mathcal{D}$ be a tensored and cotensored $\mathcal{V}$-category. For an object $L$ in $\mathcal{V}^{\Sigma_{n}}$ and a functor $\underset{\sim}{X}$ in $\operatorname{Fun}\left(\Sigma_{n} \prec \mathcal{C}^{\otimes n}, \mathcal{D}\right)$ set

$$
\left(\underset{\sim}{X} \otimes_{\Sigma_{n}} L\right)(\underline{K}):=\underset{\sim}{X}(\underline{K}) \otimes L
$$

using the tensor $\mathcal{D} \otimes \mathcal{V} \rightarrow \mathcal{D}$. This is a functor in $\underline{K}$. The symmetry automorphisms

$$
\sigma_{\sim}^{X} \otimes L: \underset{\sim}{X}\left(K_{1}, \ldots, K_{n}\right) \otimes L \rightarrow \underset{\sim}{X}\left(K_{\sigma^{-1}(1)}, \ldots, K_{\sigma^{-1}(n)}\right) \otimes L
$$

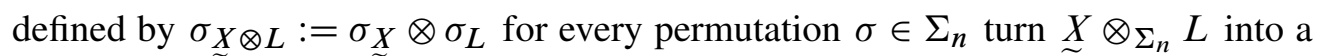
symmetric functor. For fixed $L$ in $\mathcal{V}^{\Sigma_{n}}$, the functor $\underset{\sim}{X} \mapsto \underset{\sim}{X} \otimes_{\Sigma_{n}} L$ has as a $\mathcal{V}$-right adjoint $\underset{\sim}{Y} \mapsto \operatorname{hom}_{\Sigma_{n}}(L, \underset{\sim}{Y})$, where

$$
\operatorname{hom}_{\Sigma_{n}}(L, \underset{\sim}{Y})(\underline{K}):=\operatorname{hom}_{\mathcal{D}}(L, \underset{\sim}{Y}(\underline{K})) .
$$

with symmetric structure obtained by the conjugation action.

\subsection{The cross effect}

Convention 3.8 Suppose that $\mathcal{V}=\mathcal{S}$, so that Convention 3.2 is satisfied. Suppose further that $\mathcal{D}$ is a bicomplete $\mathcal{S}$-category, and that $\mathcal{C}$ is a small $\mathcal{S}$-category with finite coproducts and terminal object $*$.

Notation 3.9 The $\mathcal{S}$-functor tr: $\mathcal{D} \rightarrow \mathcal{D}^{\Sigma_{n}}$, sending an object to itself equipped with the trivial $\Sigma_{n}$-action, has a $\mathcal{S}$-left adjoint given by the orbit functor $\left({ }_{-}\right)_{\Sigma_{n}}$.

Definition 3.10 Let $\underline{n}=\{1, \ldots, n\}$, with associated power set $P(\underline{n})$, and let

$$
P_{0}(\underline{n}):=P(\underline{n})-\{\varnothing\}
$$

be the partially ordered set of non-empty subsets of $\underline{n}$. For every $n$-tuple $\underline{K}=$ $\left\{K_{1}, \ldots, K_{n}\right\}$ of objects in $\mathcal{C}$ and every $S \in P_{0}(\underline{n})$ there is a map

$$
\bigvee_{i=1}^{n} K_{i} \rightarrow \bigvee_{i \in \underline{n}-S} K_{i}
$$


induced by the canonical inclusion $K_{i} \rightarrow \bigvee_{i \in n-S} K_{i}$ if $i \notin S$ and the trivial map $K_{i} \rightarrow *$ if $i \in S$.

Definition 3.11 The $n^{\text {th }}$ cross effect $\mathrm{cr}_{n}: \mathcal{C}^{\wedge n} \rightarrow \mathcal{D}$ of a functor $\underset{\sim}{X}: \mathcal{C} \rightarrow \mathcal{D}$ is given by the formula

$$
\operatorname{cr}_{n} \underset{\sim}{X}\left(K_{1}, \ldots, K_{n}\right):=\operatorname{fib}\left[\underset{\sim}{X}\left(\bigvee_{i=1}^{n} K_{i}\right) \rightarrow \lim _{S \in P_{0}(\underline{n})} \underset{\sim}{X}\left(\bigvee_{i \in \underline{n}-S} K_{i}\right)\right],
$$

where the map is induced by the maps described in Definition 3.10. Here "fib" refers to the strict fiber, the preimage of the basepoint.

Remark 3.12 The $n^{\text {th }}$ cross effect, as defined above, does not coincide with the construction (denoted by the same symbol) $\mathrm{cr}_{n}$ introduced by Goodwillie [11]. Goodwillie's $\mathrm{cr}_{n}$ refers to the functor

$$
\operatorname{hocr}_{n} \underset{\sim}{X}\left(K_{1}, \ldots, K_{n}\right):=\operatorname{hofib}\left[\underset{\sim}{X}\left(\bigvee_{i=1}^{n} K_{i}\right) \rightarrow \underset{S \in P_{0}(\underline{n})}{\operatorname{holim}} \underset{\sim}{X}\left(\bigvee_{i \in \underline{n}-S} K_{i}\right)\right]
$$

that we call the $n^{\text {th }}$ homotopy cross effect. Section 3.3 supplies a model structure on $\operatorname{Fun}(\mathcal{C}, \mathcal{D})$ such that the homotopy cross effect becomes the right derived functor of the strict cross effect.

A permutation $\sigma \in \Sigma_{n}$ defines an automorphism of an $n$-fold coproduct, whence $\mathrm{cr}_{n}$ produces symmetric functors

$$
\operatorname{cr}_{n}: \operatorname{Fun}(\mathcal{C}, \mathcal{D}) \rightarrow \operatorname{Fun}\left(\Sigma_{n} \prec \mathcal{C}^{\wedge n}, \mathcal{D}\right) .
$$

The main goal of this section is to construct a left adjoint to this functor.

Definition 3.13 For every object $\underline{K}=\left\{K_{1}, \ldots, K_{n}\right\}$ in $\Sigma_{n} \curlywedge \mathcal{C}^{\wedge n}$, the maps given in Definition 3.10 induce a map

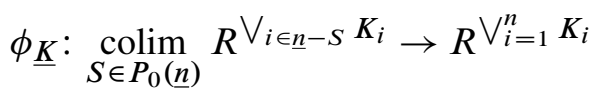

in $\operatorname{Fun}(\mathcal{C}, \mathcal{S})$, functorial in $\underline{K}$.

Lemma 3.14 For $\underline{K}$ in $\Sigma_{n} 2 \mathcal{C}^{\wedge n}$ and $\underset{\sim}{X}$ in $\operatorname{Fun}(\mathcal{C}, \mathcal{D})$ there is a canonical isomorphism

$$
\operatorname{hom}\left(\bigwedge_{i=1}^{n} R^{K_{i}}, \underset{\sim}{X}\right) \cong \operatorname{cr}_{n} \underset{\sim}{X}\left(K_{1}, \ldots, K_{n}\right) .
$$

Proof In the case $n=2$, the map $\phi_{\underline{K}}$ corresponds to the canonical map

$$
R^{K_{1}} \vee R^{K_{2}} \rightarrow R^{K_{1} \vee K_{2}} \cong R^{K_{1}} \times R^{K_{2}}
$$


whose objectwise cofiber is the objectwise smash product $R^{K_{1}} \wedge R^{K_{2}}$ described in Definition 2.8. For arbitrary $n$, the map $\phi_{\underline{K}}$ given in Definition 3.13 induces an objectwise cofiber sequence

$$
\operatorname{colim}_{S \in P_{0}(\underline{n})} R^{\bigvee_{i \in \underline{n}-S} K_{i}} \stackrel{\phi_{\underline{K}}}{\longrightarrow} R^{\bigvee_{i=1}^{n} K_{i}} \rightarrow \bigwedge_{i=1}^{n} R^{K_{i}}
$$

in $\operatorname{Fun}(\mathcal{C}, \mathcal{S})$, where $\bigwedge_{i=1}^{n} R^{K_{i}}: \mathcal{C} \rightarrow \mathcal{S}$ is the $n$-fold objectwise smash product. The result then follows from the enriched Yoneda Lemma 2.7.

Lemma 3.15 The functor

$$
\operatorname{cr}_{n}: \operatorname{Fun}(\mathcal{C}, \mathcal{D}) \rightarrow \operatorname{Fun}\left(\Sigma_{n} \curlywedge \mathcal{C}^{\wedge n}, \mathcal{D}\right)
$$

has a left $\mathcal{S}$-adjoint $\mathcal{L}_{n}$, sending the symmetric functor $\underset{\sim}{X}$ to the functor

$$
K \mapsto(\underset{\sim}{X}(K, \ldots, K))_{\Sigma_{n}}
$$

where $\Sigma_{n}$ operates by permuting the entries in $(K, \ldots, K)$.

Lemma 3.14 implies for the case $\mathcal{D}=\mathcal{S}$ that a left $\mathcal{S}$-adjoint of $\mathrm{cr}_{n}$ (if it exists) sends the functor represented by $\underline{K} \in \Sigma_{n} \prec \mathcal{C}^{\wedge n}$ to the objectwise smash product $\bigwedge_{i=1}^{n} R^{K_{i}}$. This supplies a candidate for the definition of the left adjoint by the enriched Yoneda Lemma 2.7.

Proof Let $\underset{\sim}{X}$ in $\operatorname{Fun}\left(\Sigma_{n} \prec \mathcal{C}^{\wedge n}, \mathcal{D}\right)$ be written as a colimit of representable functors:

$$
\underset{\sim}{X} \cong \int^{\underline{K}} \underset{\sim}{X}(\underline{K}) \wedge R^{K}
$$

Then the functor $\mathcal{L}_{n}: \operatorname{Fun}\left(\Sigma_{n} \curlywedge \mathcal{C}^{\wedge n}, \mathcal{D}\right) \rightarrow \operatorname{Fun}(\mathcal{C}, \mathcal{D})$ is defined by the coend

$$
\mathcal{L}_{n}(\underset{\sim}{X}) \cong \int^{\underline{K}} \underset{\sim}{X}(\underline{K}) \wedge \mathcal{L}_{n}\left(R^{\underline{K}}\right) \cong \int^{\underline{K}}\left(\underset{\sim}{X}(\underline{K}) \wedge \bigwedge_{i=1}^{n} R^{K_{i}}\right) .
$$

For every $\mathcal{S}$-functor $\underset{\sim}{Y}: \mathcal{C} \rightarrow \mathcal{D}$, one obtains natural isomorphisms

$$
\begin{aligned}
\mathcal{S}_{\text {Fun }(\mathcal{C}, \mathcal{D})}\left(\mathcal{L}_{n}(\underset{\sim}{X}), \underline{Y}\right) & \cong \mathcal{S}_{\mathrm{Fun}(\mathcal{C}, \mathcal{D})}\left(\int^{\underline{K}}\left(\underset{\sim}{X}(\underline{K}) \wedge \bigwedge_{i=1}^{n} R^{K_{i}}\right), \underset{\sim}{Y}\right) \\
& \cong \int_{\underline{K}} \mathcal{S}_{\mathcal{D}}\left(\underset{\sim}{X}(\underline{K}), \operatorname{hom}\left(\bigwedge_{i=1}^{n} R^{K_{i}}, \underset{\sim}{Y}\right)\right) \\
& \cong \int_{\underline{K}} \mathcal{S}_{\mathcal{D}}\left(\underset{\sim}{X}(\underline{K}),\left(\operatorname{cr}_{n} \underset{\sim}{Y}\right)(\underline{K})\right) \cong \mathcal{S}_{\text {Fun }\left(\Sigma_{n} \mathcal{C} \mathcal{C}^{\wedge n}, \mathcal{D}\right)}\left(\underset{\sim}{X}, \operatorname{cr}_{n} \underline{Y}\right)
\end{aligned}
$$


Lemma 3.14 is used for the third isomorphism. Hence, the functor $\mathcal{L}_{n}$ is $\mathcal{S}$-left adjoint to $\mathrm{cr}_{n}$. One identifies the functor $\mathcal{L}_{n}$ explicitly by the formula

$$
\mathcal{L}_{n} \cong\left(\Delta_{n}^{*}\left(\_\right)\right)_{\Sigma_{n}}=\underset{\operatorname{pr}_{\mathcal{C}}}{\operatorname{LKan} \circ \Delta_{n}^{*}}
$$

Here $\operatorname{pr}_{\mathcal{C}}: \mathcal{C} \times \Sigma_{n} \rightarrow \mathcal{C}$ is the projection onto the first factor, and $\Delta_{n}^{*}$ is precomposition with the symmetric diagonal $\Delta_{n}: \Sigma_{n} \times \mathcal{C} \rightarrow \Sigma_{n} 2 \mathcal{C}^{\wedge n}$ sending an object $K$ to the $n$-tuple $\Delta_{n}(K)=(K, \ldots, K)$ and a morphism $(\sigma, f)$ to $(\sigma,(f, \ldots, f))$. A straightforward computation shows that the right-hand side of (3-2) sends the functor represented by $\underline{K} \in \Sigma_{n} \prec \mathcal{C}^{\wedge n}$ to the objectwise smash product $\bigwedge_{i=1}^{n} R^{K_{i}}$. Since it also commutes with colimits, the isomorphism (3-2) holds by the universal property of the left Kan extension. This supplies the formula stated in the Lemma.

\subsection{The cross effect model structure}

Suppose that $\mathcal{D}$ is a cofibrantly generated $\mathcal{S}$-model category, so that Theorem 2.14 is applicable. In all interesting cases, the cross effect

$$
\operatorname{cr}_{n}: \operatorname{Fun}(\mathcal{C}, \mathcal{D})_{\text {proj }} \rightarrow \operatorname{Fun}\left(\Sigma_{n} \prec(\mathcal{C})^{\wedge n}, \mathcal{D}\right)_{\text {proj }}
$$

is not a right Quillen functor of projective model structures. One can deduce this from the behavior of the second homotopy cross effect, which measures the failure of linearity for reduced homotopy functors. For example, the $\mathcal{S}$-functor $\underset{\sim}{Q}:=\operatorname{Sing} \circ|-|: \mathcal{S} \rightarrow \mathcal{S}$ sending a pointed simplicial set to the singular complex of its geometric realization is an objectwise fibrant replacement of the identity functor $\operatorname{Id}_{\mathcal{S}}$. As the functor $\operatorname{Id}_{\mathcal{S}}$ is not linear, its homotopy cross effect $\operatorname{hocr}_{2}\left(\operatorname{Id}_{\mathcal{S}}\right) \simeq \operatorname{hocr}_{2}(\underset{\sim}{Q)})$ is not contractible. However, since the canonical map

$$
\underset{\sim}{Q}(K \vee L) \rightarrow \underset{\sim}{Q}(K \times L) \cong \underset{\sim}{Q}(K) \times \underset{\sim}{Q}(L)
$$

is injective for all $K, L \in \mathcal{S}$, one has $\operatorname{cr}_{2} Q=\underset{\sim}{*}$. The purpose of this section is to supply a model structure on $\operatorname{Fun}(\mathcal{C}, \mathcal{D})$ with objectwise weak equivalences, such that the $n^{\text {th }}$ cross effect is a right Quillen functor. The task will be accomplished by introducing more cofibrations. The right derived functor of the cross effect turns out to be Goodwillie's homotopy cross effect, as promised in Remark 3.12.

Definition 3.16 Let $n \geq 1$. If $\underline{K}=\left\{K_{1}, \ldots, K_{n}\right\}$ is an $n$-tuple of objects in $\mathcal{C}$, one says $|\underline{K}|=n$. Definition 3.13 supplies a map

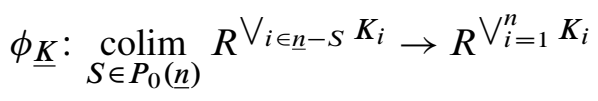

in $\operatorname{Fun}(\mathcal{C}, \mathcal{S})$ for every $\underline{K}$ with $|\underline{K}|=n$. Let $\Phi_{n}:=\left\{\phi_{\underline{K}}|| \underline{K} \mid \leq n\right\}$, and $\Phi:=\Phi_{\infty}:=$ $\bigcup_{n \geq 1} \Phi_{n}$. 
The argument with $\underset{\sim}{Q}$ given above indicates that not all of the maps in $\Phi_{2}$ are projective cofibrations.

Definition 3.17 The tensor $\mathcal{S} \times \mathcal{D} \rightarrow \mathcal{D}$ induces a tensor $\operatorname{Fun}(\mathcal{C}, \mathcal{S}) \times \mathcal{D} \rightarrow \operatorname{Fun}(\mathcal{C}, \mathcal{D})$. Hence, the pushout product $\square$ of Definition 2.10 of a map in $\operatorname{Fun}(\mathcal{C}, \mathcal{S})$ and a map in $\mathcal{D}$ is defined. For $1 \leq n \leq \infty$, the pushout product defines two sets of maps in $\operatorname{Fun}(\mathcal{C}, \mathcal{D})$ :

$$
I_{n}^{\mathrm{cr}}=\left(\Phi_{n} \square I_{\mathcal{D}}\right) \quad J_{n}^{\mathrm{cr}}=\left(\Phi_{n} \square J_{\mathcal{D}}\right)
$$

Set $I^{\mathrm{cr}}:=I_{\infty}^{\mathrm{cr}}$ and $J^{\mathrm{cr}}:=J_{\infty}^{\mathrm{cr}}$. A map in $\operatorname{Fun}(\mathcal{C}, \mathcal{D})$ is:

(1) A cross effect fibration or cr fibration if it belongs to the class $J^{\text {cr }}$-inj, that is, it has the right lifting property with respect to all maps in $J^{\text {cr }}$.

(2) A cross effect cofibration or cr cofibration if it belongs to the class $I^{\mathrm{cr}}$-cof, that is, it has the left lifting property with respect to all cr fibrations.

Weak equivalences are still given by objectwise weak equivalences.

Remark 3.18 Note that $I_{1}^{\mathrm{cr}}=I_{\mathrm{Fun}(\mathcal{C}, \mathcal{D})}^{\mathrm{proj}}$ and $J_{1}^{\mathrm{cr}}=J_{\text {Fun }(\mathcal{C}, \mathcal{D})}^{\text {proj }}$. In particular, every projective cofibration is a cr cofibration and every acyclic projective cofibration is an acyclic cr cofibration.

Theorem 3.19 The classes of objectwise weak equivalences, cross effect fibrations and cross effect cofibrations form a cofibrantly generated $\mathcal{S}$-model structure on the category $\operatorname{Fun}(\mathcal{C}, \mathcal{D})$, which is as proper as $\mathcal{D}$.

The idea for the proof of the theorem can be found in Isaksen [14]. It is an application of the recognition principle for cofibrantly generated model structures (see Hirschhorn [12, 11.3.1]). Replacing $\Phi$ with $\Phi_{n}$ for some $1 \leq n \leq \infty$ leads to a model structure where only the $k^{\text {th }}$ cross effects for $1 \leq k \leq n$ become right Quillen functors. The model structure supplied by Theorem 3.19 is called the cross effect model structure or simply cr model structure and denoted by $\operatorname{Fun}(\mathcal{C}, \mathcal{D})_{\mathrm{cr}}$.

Proof Remark 3.18 implies that every projective cofibration is a cr cofibration. Sources and targets of maps in $I^{\text {cr }}$ and $J^{\text {cr }}$ are as small as the sources and targets of the maps in $I_{\mathcal{D}}$ and $I_{\mathcal{D}}$, thus allowing the small object argument. Lemmata 3.20, 3.21 and 3.22 below conclude the proof of the existence of the cofibrantly generated model structure. The model structure is an $\mathcal{S}$-model structure, as one checks on the generators $I^{\text {cr }}$ and $J^{\text {cr }}$. The statement regarding properness is proved in Lemma 3.23. 
Lemma 3.20 A map in $\operatorname{Fun}(\mathcal{C}, \mathcal{D})$ is in $I^{\mathrm{cr}}$-inj if and only if it is an objectwise weak equivalence and a cross effect fibration.

Proof Let $f: \underset{\sim}{X} \rightarrow \underset{\sim}{Y}$ be in $I^{\text {cr }}-$ inj. Then $f$ is in $I_{\text {Fun }(\mathcal{C}, \mathcal{D})}^{\text {proinj, and so an acyclic }}$ projective fibration. In particular, $f$ is an objectwise weak equivalence. The inclusion $I_{\mathcal{D}}$-inj $\subset J_{\mathcal{D}}$-inj implies

$$
\left(\Phi \square I_{\mathcal{D}}\right)-\text { inj } \subset\left(\Phi \square J_{\mathcal{D}}\right)-\text { inj },
$$

whence $f$ is a cr fibration as well.

Conversely, let $f$ be an objectwise equivalence and a cr fibration. Then it is in particular an acyclic projective fibration, hence in $I_{\operatorname{Fun}(\mathcal{C}, \mathcal{D})}^{\text {proj }}-$ inj. By assumption, it is also in $\left(\Phi \square J_{\mathcal{D}}\right)$-inj, which means exactly that the map

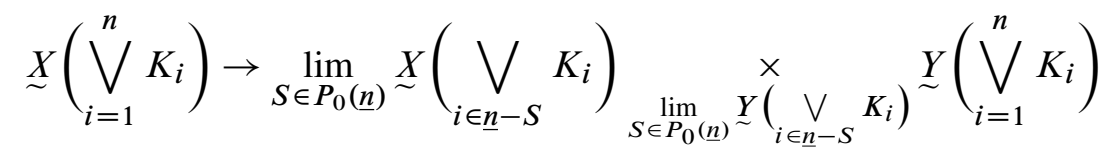

is a fibration for every possible choice of $n$ and $\underline{K}$. It remains to show that $f$ is in ( $\Phi \square I_{\mathcal{D}}$ )-inj. This is equivalent to the map in (3-3) being an acyclic fibration. By assumption, the map

$$
\underset{\sim}{X}\left(\bigvee_{i=1}^{n} K_{i}\right) \rightarrow \underset{\sim}{Y}\left(\bigvee_{i=1}^{n} K_{i}\right)
$$

is a weak equivalence. Thus it suffices to show that the map

$$
\lim _{S \in P_{0}(\underline{n})} \underset{\sim}{X}\left(\bigvee_{i \in \underline{n}-S} K_{i}\right) \rightarrow \lim _{S \in P_{0}(\underline{n})} \underset{Y}{Y}\left(\bigvee_{i \in \underline{n}-S} K_{i}\right)
$$

is an acyclic fibration. To conclude this, recall that $P_{0}(\underline{n})$ is an inverse category by the functor deg: $P_{0}(\underline{n})^{\text {op }} \rightarrow \mathbb{N}$ which sends $S$ to the number of elements in $\underline{n} \backslash S$. By Hovey [13, Theorem 5.1.3], there is a model structure on the category of functors from $P_{0}(\underline{n})$ to any model category. It has objectwise weak equivalences and cofibrations, and the fibrations are characterized by an appropriate matching space condition. The limit is thus a right Quillen functor on this functor category, and in particular preserves acyclic fibrations. Since $f: \underset{\sim}{X} \rightarrow \underset{\sim}{Y}$ is a cr fibration, the induced natural transformation $f^{\prime}$ of functors on $P_{0}(\underline{n})$ is a fibration. As $f$ is an objectwise equivalence, $f^{\prime}$ is an objectwise weak equivalence. The result follows.

Lemma 3.21 A map in $J^{\text {cr }}$-cof is a cr cofibration. 
Proof If a map is in $J^{\text {cr }}$-cof, it has the left lifting property with respect to all cr fibrations. Thus it has the left lifting property with respect to all cr fibrations that are also objectwise weak equivalences. So, by Lemma 3.20, it is a cr cofibration.

Lemma 3.22 A map in $J^{\text {cr }}$-cof is an objectwise weak equivalence.

Proof By the small object argument, every map in $J^{\text {cr }}$-cof is a retract of a map in $J^{\text {cr }}$-cell. Since $\mathcal{V}=\mathcal{S}$, every map in $\Phi$ is an objectwise cofibration in $\operatorname{Fun}(\mathcal{C}, \mathcal{S})$. This implies that every map in $J^{\text {cr }}$-cell is an objectwise weak equivalence.

Lemma 3.23 If $\mathcal{D}$ is right or left proper, then the cross effect model structure is right or left proper, respectively.

Proof Any cr fibration is an objectwise fibration and any cr cofibration is an objectwise cofibration, again using $\mathcal{V}=\mathcal{S}$ for the latter statement. Since pullbacks and pushouts are formed objectwise, the statement follows.

Lemma 3.24 If the functor $\underset{\sim}{X}$ is cross effect fibrant, the canonical map

$$
\operatorname{cr}_{n} \underset{\sim}{X} \rightarrow \operatorname{hocr}_{n} \underset{\sim}{X}
$$

is an objectwise weak equivalence.

Proof If $\underset{\sim}{X}$ is cr fibrant, then - as in the proof of Lemma 3.20 - the $P_{0}(\underline{n})$-diagram

$$
S \mapsto \underset{\sim}{X}\left(\bigvee_{i \in \underline{n}-S} K_{i}\right)
$$

is injectively fibrant. This follows from the right lifting property of the map $\underset{\sim}{X} \rightarrow *$ with respect to $J_{m}^{\mathrm{cr}}$ for $1 \leq m<n$. Thus, the map

$$
\lim _{S \in P_{0}(\underline{n})} \underset{\sim}{X}\left(\bigvee_{i \in \underline{n}-S} K_{i}\right) \rightarrow \operatorname{holim}_{S \in P_{0}(\underline{n})} \underset{\sim}{X}\left(\bigvee_{i \in \underline{n}-S} K_{i}\right)
$$

is a weak equivalence. The right lifting property with respect to $J_{n}^{\text {cr }}$ implies that the map

$$
\underset{\sim}{X}\left(\bigvee_{i=1}^{n} K_{i}\right) \rightarrow \lim _{S \in P_{0}(\underline{n})} \underset{\sim}{X}\left(\bigvee_{i \in \underline{n}-S} K_{i}\right)
$$

is a fibration. The claim follows. 
Proposition 3.25 The functor

$$
\operatorname{cr}_{n}: \operatorname{Fun}(\mathcal{C}, \mathcal{D})_{\mathrm{cr}} \rightarrow \operatorname{Fun}\left(\Sigma_{n} \prec(\mathcal{C})^{\wedge n}, \mathcal{D}\right)_{\text {proj }}
$$

is a right Quillen functor and hocr $_{n}$ is its right derived functor.

Proof Let $\underline{K}=\left(K_{1}, \ldots, K_{n}\right)$ be an $n$-tuple of objects in $\mathcal{C}$. The cofiber sequence

$$
\underset{S \in P_{0}(\underline{n})}{\operatorname{colim}} R^{\bigvee_{i \in \underline{n}-S} K_{i}} \stackrel{\phi_{\underline{K}}}{\longrightarrow} R^{\bigvee_{i=1}^{n} K_{i}} \longrightarrow \bigwedge_{i=1}^{n} R^{K_{i}}
$$

in $\operatorname{Fun}(\mathcal{C}, \mathcal{S})$ implies that the functor $\bigwedge_{i=1}^{n} R^{K_{i}}$ is cr cofibrant, because the map $\phi_{\underline{K}}$ is a cr cofibration. By Lemma 3.14,

$$
\operatorname{hom}\left(\bigwedge_{i=1}^{n} R^{K_{i}}, \underset{\sim}{X}\right) \cong \operatorname{cr}_{n} \underset{\sim}{X}\left(K_{1}, \ldots, K_{n}\right),
$$

the strict cross effect is a right Quillen functor. Its right derived functor hocr $_{n}$ is identified by Lemma 3.24.

\section{Homotopy functors}

Definition 4.1 Suppose $\mathcal{B}$ and $\mathcal{D}$ are model categories and $\mathcal{C}$ is a small full subcategory of $\mathcal{B}$. A functor in $\operatorname{Fun}(\mathcal{C}, \mathcal{D})$ is called a homotopy functor if for every weak equivalence $A \rightarrow B$ in $\mathcal{C}$ the image $F(A) \rightarrow F(B)$ is a weak equivalence in $\mathcal{D}$.

Homotopy functors are the main object of study in Goodwillie's calculus of functors. From the point of view of model categories, the full subcategory of homotopy functors is usually inadequate. The aim of this section is to construct a model structure in which every functor is a homotopy functor, up to weak equivalence.

\subsection{Homotopy functors and simplicial functors}

A preliminary goal is to show that every homotopy functor of reasonable categories is objectwise weakly equivalent to a simplicial functor. The following statement is a slight generalization of a lemma by Waldhausen [22, Lemmata 3.1.2 and 3.1.3] to certain $\mathcal{U}$-model categories.

Lemma 4.2 Let $\mathcal{C}$ be a small subcategory of a simplicial model category, closed under cotensoring with finite simplicial sets, and let $\mathcal{D}$ be a $\mathcal{U}$-model category. Suppose that $X: \mathcal{C} \rightarrow \mathcal{D}$ is a homotopy functor. If the simplicial object $n \mapsto X\left(A^{\Delta^{n}}\right)$ is Reedy cofibrant for every object $A \in \mathcal{C}$, then there exists a $\mathcal{U}$-functor $\underset{\sim}{X}: \mathcal{C} \rightarrow \mathcal{D}$ and a natural objectwise weak equivalence $f: X \rightarrow \underset{\sim}{X}$. 
Proof The value of the functor $\underset{\sim}{X}$ at an object $A$ of $\mathcal{C}$ is defined as the coend

$$
\underset{\sim}{X}(A):=\int^{n} X\left(A^{\Delta^{n}}\right) \times \Delta^{n},
$$

which is in fact the standard realization of a simplicial object in $\mathcal{D}$. Expressing $X(A)$ as the standard realization of a constant simplicial object, one obtains a natural transformation $f: X \rightarrow \underset{\sim}{X}$ via $\Delta^{n} \rightarrow \Delta^{0}$ :

$$
f(A): X(A) \cong \int^{n} X\left(A^{\Delta^{0}}\right) \times \Delta^{n} \rightarrow \int^{n} X\left(A^{\Delta^{n}}\right) \times \Delta^{n}=\underset{\sim}{X}(A) .
$$

The map $A=A^{\Delta^{0}} \rightarrow A^{\Delta^{n}}$ is a simplicial homotopy equivalence, since $\Delta^{n} \rightarrow \Delta^{0}$ is one. It follows that it is a weak equivalence in $\mathcal{C}$. Hence, so is its image under $X$ by assumption. The constant simplicial object $X(A)$ is cofibrant in the Reedy model structure. Since by assumption the target of $f(A)$ is Reedy cofibrant as well, $f$ is a natural weak equivalence. The reason is that realization is a left Quillen functor on the Reedy model structure.

It remains to prove that $\underset{\sim}{X}$ is a $\mathcal{U}$-functor. A map of simplicial sets

$$
\mathcal{U}_{\mathcal{C}}(A, B) \rightarrow \mathcal{U}_{\mathcal{D}}(\underset{\sim}{X}(A), \underset{\sim}{X}(B))
$$

will be given in simplicial degree $m$ as follows. An $m$-simplex $A \times \Delta^{m} \rightarrow B$ can equivalently be described as a map $\alpha: A \rightarrow B^{\Delta^{m}}$. Consider the simplicial objects $[n] \mapsto F_{n}=X\left(A^{\Delta^{n}}\right),[n] \mapsto G_{n}=X\left(B^{\Delta^{n}}\right)$. An $m$-simplex $F_{\bullet} \rightarrow G_{\bullet}$ is the same as a natural transformation

$$
(\gamma:[n] \rightarrow[m]) \mapsto\left(t_{\gamma}: F_{n} \rightarrow G_{n}\right) .
$$

Set $t_{\gamma}$ to be the composition

$$
X\left(A^{\Delta^{n}}\right) \stackrel{X\left(\alpha^{\Delta^{n}}\right)}{\longrightarrow} X\left(B^{\Delta^{m} \times \Delta^{n}}\right) \stackrel{X\left(B^{(\gamma, \mathrm{id})}\right)}{\longrightarrow} X\left(B^{\Delta^{n}}\right)
$$

which induces the desired map

$$
\underset{\sim}{X}(A) \times \Delta^{m} \rightarrow \underset{\sim}{X}(B) .
$$

The verification of the relevant axioms this map has to fulfill is left to the reader.

Note that the condition on Reedy cofibrancy is fulfilled automatically in many cases, for example in the category of simplicial presheaves with the injective model structure.

Remark 4.3 Recall from Definition 2.17 that a functor $X$ between pointed categories is reduced if $X(*) \cong *$. A $\mathcal{U}$-functor is an $\mathcal{S}$-functor if and only it is reduced. Hence, 
the analog of Lemma 4.2 for $\mathcal{S}$-model categories and reduced homotopy functors holds as well. In fact, one can replace a homotopy functor with $X(*) \simeq *$ by a weakly equivalent $\mathcal{S}$-functor.

\subsection{A model structure for simplicial homotopy functors}

The purpose of this section is to construct a model structure on a category of enriched functors in which every enriched functor is weakly equivalent to an enriched homotopy functor. For specific categories of enriched functors this has been obtained by Lydakis [18], Dundas, Röndigs and Østvær [7] and Biedermann, Chorny and Röndigs [3]. Although more general results are possible, a restriction to simplicial functors seems adequate, as Lemma 4.2 suggests. None of this is necessary if all functors are already homotopy functors, as it is the case if the source category is the category of finite CW complexes, or more generally consists of bifibrant objects only. This section is written in the pointed setting. All statements in this section and their proofs have unpointed variants, whose formulation is left to the reader.

Definition 4.4 An $\mathcal{S}$-model category $\mathcal{B}$ has a decent fibrant replacement functor if there exists a $\mathcal{S}$-natural transformation

$$
\phi_{\text {Fibr }}: \operatorname{Id}_{\mathcal{B}} \rightarrow \text { Fibr }
$$

of $\mathcal{S}$-functors satisfying the following conditions:

(1) For every object $A \in \mathcal{B}$ the object $\operatorname{Fibr}(A)$ is fibrant and $\phi_{\text {Fibr }}(A)$ is an acyclic cofibration.

(2) The functor Fibr sends weak equivalences of cofibrant objects to simplicial homotopy equivalences.

(3) The functor Fibr commutes with filtered colimits.

Example 4.5 In the case $\mathcal{B}=\mathcal{S}$ or $\mathcal{U}$ one can use Kan's $\mathrm{Ex}^{\infty}$, as well as the composition of the geometric realization and the singular complex, as a decent fibrant replacement functor.

Lemma 4.6 Let $\mathcal{B}$ be an $\mathcal{S}$-model category. Suppose there exists a set

$$
\{j: s j \rightarrow t j\}_{j \in J}
$$

of acyclic cofibrations in $\mathcal{B}$ with the following properties:

(1) An object $A \in \mathcal{B}$ is fibrant if $A \rightarrow *$ has the right lifting property with respect to $J$. 
(2) The functor $\mathcal{S}_{\mathcal{B}}(s j,-)=R^{s j}: \mathcal{B} \rightarrow \mathcal{S}$ commutes with filtered colimits for every $j \in J$.

Then $\mathcal{B}$ has a decent fibrant replacement functor.

Proof This is an enriched version of Quillen's small object argument, as constructed by Dundas, Röndigs and Østvær [7]. Let Fibr $_{1}$ be the $\mathcal{S}$-functor defined as the pushout of

$$
\bigvee_{j \in J} R^{s j} \wedge t j \longleftarrow \bigvee_{j \in J} R^{s j} \wedge s j \longrightarrow \mathrm{Id}_{\mathcal{B}}
$$

It comes together with an $\mathcal{S}$-natural transformation $\phi_{1}: \operatorname{Id}_{\mathcal{B}} \rightarrow$ Fibr $_{1}$. For $n \geq 1$ set $\operatorname{Fibr}_{n+1}=\operatorname{Fibr}_{1} \circ \mathrm{Fibr}_{n}$ and let

$$
\text { Fibr }=\operatorname{colim}\left(\operatorname{Id}_{\mathcal{B}} \stackrel{\phi_{1}}{\longrightarrow} \text { Fibr }_{1} \stackrel{\phi_{1} \circ \mathrm{Fibr}_{1}}{\longrightarrow} \operatorname{Fibr}_{2} \rightarrow \cdots\right)
$$

The natural transformation $A \rightarrow \operatorname{Fibr}(A)$ is then an acyclic cofibration. Since every $s j$ is in particular finitely presentable, a morphism $\alpha: s j \rightarrow \operatorname{Fibr}(A)$ factors over $\operatorname{Fibr}_{n}(A)$. The composition

$$
t j \cong S^{0} \wedge t j \rightarrow \bigvee_{j \in J} \mathcal{S}_{\mathcal{B}}\left(s j, \operatorname{Fibr}_{n}(A)\right) \wedge t j \rightarrow \operatorname{Fibr}_{n+1}(A) \rightarrow \operatorname{Fibr}(A)
$$

solves the lifting problem given by $\alpha$. Thus $\operatorname{Fibr}(A)$ is fibrant. Weak equivalences of bifibrant objects in an $\mathcal{S}$-model category are simplicial homotopy equivalences by Hirschhorn [12, Section 9.5]. Thus it remains to prove the third condition. It follows because Fibr $_{1}$ is a colimit of functors preserving filtered colimits by definition.

Convention 4.7 In addition to Convention 3.8, the following statements are assumed to be true:

(1) The category $\mathcal{C}$ is a small full sub- $\mathcal{S}$-category of an $\mathcal{S}$-model category $\mathcal{B}$, containing only $\mathcal{S}$-finitely presentable cofibrant objects.

(2) There exists a decent fibrant replacement functor $\operatorname{Id}_{\mathcal{B}} \rightarrow$ Fibr such that, for every $A \in \mathcal{C}$, the object $\operatorname{Fibr}(A) \in \mathcal{B}$ is a filtered colimit of objects in $\mathcal{C}$.

(3) The category $\mathcal{D}$ is a right proper cofibrantly generated $\mathcal{S}$-model category.

(4) In $\mathcal{D}$, weak equivalences, fibrations with fibrant codomain, and pullbacks are preserved under filtered colimits. 
A sufficient condition on the model category $\mathcal{D}$ to satisfy Convention 4.7(4) is essentially due to Voevodsky.

Definition 4.8 (Dundas, Röndigs and Østvær [7, 3.4]) A cofibrantly generated model category $\mathcal{D}$ is called weakly finitely generated if we can choose a set of generating cofibrations $I$ and of generating acyclic cofibrations $J$ such that the following conditions hold:

(1) The domains and codomains of the maps in $I$ are finitely presentable.

(2) The domains of the maps in $J$ are small.

(3) There exists a subset $J^{\prime}$ of $J$ of maps with finitely presentable domains and codomains such that a map in $\mathcal{D}$ with fibrant codomain is a fibration if and only if it is in $J^{\prime}-$ inj.

If $\mathcal{D}$ is a locally finitely presentable category, then pullbacks are preserved under filtered colimits in $\mathcal{D}$. The remaining requirements listed in Convention 4.7(4) are met, as proved in [7, Lemma 3.5], if the model structure on $\mathcal{D}$ is weakly finitely generated. The class of weakly finitely generated model structures is closed under left Bousfield localization with respect to a set of morphisms with finitely presentable cofibrant (co)domains.

Suppose from now on that Convention 4.7 holds. The first condition of Convention 4.7 has the following consequence.

Lemma 4.9 Let $i: \mathcal{C} \rightarrow \mathcal{B}$ be the inclusion functor. Every $\mathcal{S}$-functor $\underset{\sim}{X}: \mathcal{C} \rightarrow \mathcal{D}$ admits a left $\mathcal{S}-$ Kan extension $i_{*}(\underset{\sim}{X}): \mathcal{B} \rightarrow \mathcal{D}$, and the latter preserves filtered colimits.

Proof Convention 4.7(1) implies that every $\mathcal{S}$-functor $\underset{\sim}{Y}: \mathcal{B} \rightarrow \mathcal{D}$ which is represented by an object of $\mathcal{C}$ preserves filtered colimits. Suppose $\underset{\sim}{X}: \mathcal{C} \rightarrow \mathcal{D}$ is an $\mathcal{S}$-functor. By construction, the left $\mathcal{S}$-Kan extension $i_{*}(\underset{\sim}{X})$ is a colimit of $\mathcal{S}$-functors represented by objects of $\mathcal{C}$, which gives the result.

Definition 4.10 Let $\underset{\sim}{X}: \mathcal{C} \rightarrow \mathcal{D}$ be an $\mathcal{S}$-functor, and let

$$
\phi_{\underset{\sim}{X}}: \underset{\sim}{X} \rightarrow \underset{\sim}{X}{ }^{\mathrm{hf}}:=i_{*}(\underset{\sim}{X}) \circ \text { Fibr } \circ i
$$

denote the canonical map to the composition. The composition $\underset{\sim}{X}$ hf: $\mathcal{C} \rightarrow \mathcal{D}$ is again an $\mathcal{S}$-functor. 
Definition 4.11 A map $f: \underset{\sim}{X} \rightarrow \underset{\sim}{Y}$ is

(1) an $h f$ equivalence if the map $f^{\mathrm{hf}}:{\underset{\sim}{X}}^{\mathrm{hf}} \rightarrow{\underset{\sim}{Y}}^{\mathrm{hf}}$ is an objectwise weak equivalence.

(2) an hf fibration if it is an objectwise fibration $\underset{\sim}{X} \rightarrow \underset{\sim}{Y}$ such that the square

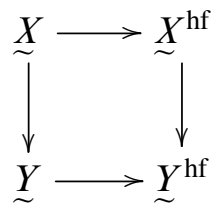

is an objectwise homotopy pullback square.

The hf cofibrations are the projective cofibrations. Theorem 4.14 states that these classes form a model structure on $\operatorname{Fun}(\mathcal{C}, \mathcal{D})$. It is called the homotopy functor model structure or $h f$ model structure for short, and denoted $\operatorname{Fun}(\mathcal{C}, \mathcal{D})_{\mathrm{hf}}$. Analogous definitions can be given starting from the cross effect model structure instead of the projective model structure. The resulting model category is denoted $\operatorname{Fun}(\mathcal{C}, \mathcal{D})_{\mathrm{hf}-\mathrm{cr}}$.

Remark 4.12 In an $\mathcal{S}$-model category, any simplicial homotopy equivalence is in particular a weak equivalence. Simplicial homotopy equivalences - unlike weak equivalences - are preserved by any $\mathcal{S}$-functor.

Remark 4.13 A few words about hf fibrant $\mathcal{S}$-functors:

(1) A (cr) fibrant functor $\underset{\sim}{X}$ is (cr) hf fibrant if and only if the map (4-1) is an objectwise weak equivalence.

(2) The functor $\underset{\sim}{X}{ }^{\mathrm{hf}}$ preserves weak equivalences. Thus a (cr) hf fibrant functor preserves weak equivalences.

(3) A (cr) fibrant functor preserving weak equivalences is (cr) hf fibrant.

(4) The hf fibrant functors are exactly the objectwise fibrant homotopy functors. The $\mathrm{hf}$ cr fibrant functors are exactly the cr fibrant homotopy functors.

Theorem 4.14 Assume Convention 4.7. The classes of maps given in Definition 4.11, starting from the projective or the cross effect model structure, constitute a right proper cofibrantly generated $\mathcal{S}$-model structure. It is left proper if $\mathcal{D}$ is left proper.

Proof As in our previous article [3], it suffices to check that the natural transformation $\phi_{X}: \underset{\sim}{X} \rightarrow \underset{\sim}{X}{ }^{\mathrm{hf}}$ satisfies the axioms (A1), (A2), and (A3) given by Bousfield in [5, 9.2]. Cofibrant generation is delegated to Lemma 4.16. 
Axiom (A1) Let $f: \underset{\sim}{X} \rightarrow \underset{\sim}{Y}$ be an objectwise weak equivalence. To prove that $f^{\text {hf }}$ is an objectwise weak equivalence, let $A \in \mathcal{C}$ and express $\operatorname{Fibr}(A)$ as a filtered colimit of objects $B_{i}$ in $\mathcal{C}$ by Convention 4.7(2). Lemma 4.9 implies that $f^{\text {hf }}(A)$ is the morphism induced on filtered colimits by the morphisms $f\left(B_{i}\right)$, which are weak equivalences. Convention 4.7(4) implies that $f^{\mathrm{hf}}(A)$ is itself a weak equivalence.

Axiom (A2) The task is to identify the two natural transformations

$$
\phi_{\sim}^{X}{ }_{\sim}^{\mathrm{hf}}, \phi_{\sim}^{\mathrm{hf}}: \underset{\sim}{X}{ }^{\mathrm{hf}} \rightarrow\left({\underset{\sim}{X}}^{\mathrm{hf}}\right)^{\mathrm{hf}}
$$

as weak equivalences. The triangular identities and the natural isomorphism

$$
\underset{\sim}{X} \stackrel{\cong}{\rightarrow} i_{*}(\underset{\sim}{X}) \circ i
$$

reduce the problem to the value of the two natural maps

$$
\operatorname{Fibr}(A) \rightarrow \operatorname{Fibr}(\operatorname{Fibr}(A))
$$

under the $\mathcal{S}$-functor $\underset{\sim}{X}$. Both maps are simplicial homotopy equivalences, since Fibr is a decent fibrant replacement functor and all objects in $\mathcal{C}$ are cofibrant by Convention 4.7(1). Remark 4.12 then implies that the maps in question are objectwise weak equivalences.

Axiom (A3) Let $f: \underset{\sim}{X} \rightarrow \underset{\sim}{Y}$ be an hf weak equivalence and let $p: \underset{\sim}{Z} \rightarrow \underset{\sim}{Y}$ be an objectwise fibration with $\underset{\sim}{Y}$ objectwise fibrant; cr fibrations are not necessary. Consider the pullback diagram

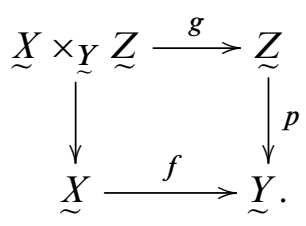

The goal is to prove that $g^{\text {hf }}$ is an objectwise weak equivalence. Pullbacks are computed objectwise. Lemma 4.9 and Convention 4.7(4) imply that the diagram

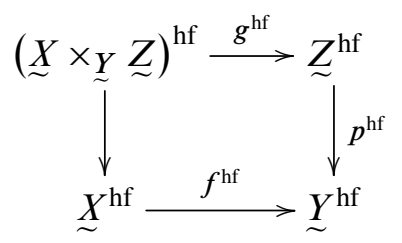

is a pullback diagram. Moreover, since fibrations in $\mathcal{D}$ with fibrant target are closed under filtered colimits, $p^{\text {hf }}$ is still an objectwise fibration. Now $f^{\text {hf }}$ is an objectwise weak equivalence by assumption and $\mathcal{D}$ is right proper. Thus, $g^{\text {hf }}$ is an objectwise weak equivalence, which finishes the proof. 
Lemma 4.15 Let $p: \underset{\sim}{X} \rightarrow \underset{\sim}{Y}$ be an objectwise fibration. Then the following statements are equivalent:

(i) The map $p$ is an hf fibration.

(ii) The induced square

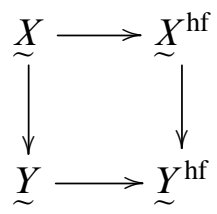

is an objectwise homotopy pullback.

(iii) For each weak equivalence $A \rightarrow B$ in $\mathcal{C}$ the induced square

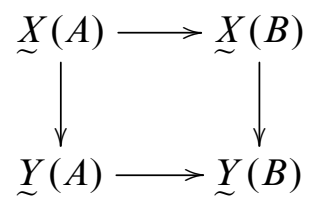

is a homotopy pullback square.

The corresponding statement for cr fibrations holds as well.

Proof The equivalence of (i) and (ii) follows from Bousfield's characterization [5, Theorem 9.3] of fibrations in the localized model structure. The equivalence of (ii) and (iii) will be shown now. Let $\underset{\sim}{X} \rightarrow \underset{\sim}{Y}$ satisfy (ii). Since the functors $\underset{\sim}{X \text { hf }}$ and $\underset{\sim}{Y \text { hf }}$ are homotopy functors, the induced diagram

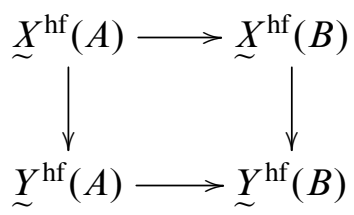

is a homotopy pullback diagram for any weak equivalence $A \rightarrow B$ in $\mathcal{C}$ for trivial reasons. This means that the composed outer square and the right-hand square in the following diagram are homotopy pullbacks:

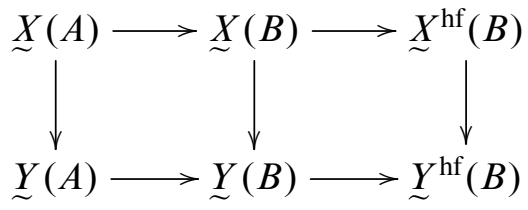

It follows that the left-hand square is a homotopy pullback. 
Now let $\underset{\sim}{X} \rightarrow \underset{\sim}{Y}$ satisfy property (iii), and let $A \rightarrow B$ be a weak equivalence in $\mathcal{C}$. Consider a decent fibrant replacement $\phi_{A}: A \rightarrow \operatorname{Fibr}(A)$. By Convention 4.7(2), this map factors through a colimit

$$
A \rightarrow \cdots \rightarrow B_{i} \rightarrow B_{i+1} \rightarrow \cdots \rightarrow \operatorname{Fibr}(A)
$$

where all objects $B_{i}$ are in $\mathcal{C}$. By (iii) there are homotopy pullback diagrams:

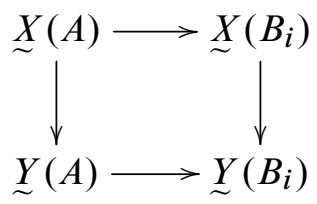

Their colimit yields the desired homotopy pullback in (ii) since homotopy pullbacks commute with filtered colimits in $\mathcal{D}$ by Convention 4.7(4).

Lemma 4.16 Assume Convention 4.7. Then the homotopy functor model structure is cofibrantly generated.

Proof In order to enlarge the set of generating acyclic cofibrations of the $\mathrm{cr}$ or projective model structure, respectively, take an arbitrary weak equivalence $w: A \stackrel{\simeq}{\rightarrow} B$ in $\mathcal{C}$. It induces the map

$$
w^{*}: R^{B} \rightarrow R^{A}
$$

which, using the simplicial mapping cylinder construction, can be factored as a projective cofibration $w \prime$, followed by a simplicial homotopy equivalence. The additional set of generating acyclic cofibrations is

$$
\left\{w^{\prime} \square i\right\}
$$

where $i$ runs through a set $I_{\mathcal{D}}$ of generating cofibrations of $\mathcal{D}$ and $w$ runs through the set of weak equivalences in $\mathcal{C}$. The fact that hf fibrations are exactly those objectwise fibrations with the right lifting property with respect to this set follows from Lemma 4.15.

Remark 4.17 It is now clear that the $(\mathrm{cr})$ hf model structure on $\operatorname{Fun}(\mathcal{C}, \mathcal{D})$ can also be viewed as the left Bousfield localization of the (cr) projective model structure with respect to the set

$$
\left\{R^{B} \rightarrow R^{A} \mid A \rightarrow B \text { is a weak equivalence in } \mathcal{C}\right\} .
$$




\subsection{Homotopy functors in several variables}

Recall that in the product of $\mathcal{S}$-model categories $\mathcal{B}_{1} \times \cdots \times \mathcal{B}_{n}$ a morphism $f: \underline{K} \rightarrow \underline{L}$ is a weak equivalence (or fibration, or cofibration) if every component $K_{i} \rightarrow L_{i}$ is so in $\mathcal{C}_{i}$. This defines an $\mathcal{S}$-model structure. In particular, Definition 4.1 is applicable in Fun $\left(\mathcal{C}_{1} \times \cdots \times \mathcal{C}_{n}, \mathcal{D}\right)$, where $\mathcal{C}_{k} \subset \mathcal{B}_{k}$ is a full subcategory for every $k \in \underline{n}$. Note that a functor in the product category is a homotopy functor if and only if all its partial functors are homotopy functors. The results of Section 4.2 apply.

Corollary 4.18 Assume that for all $k \in \underline{n}$ the categories $\mathcal{C}_{i} \subset \mathcal{B}_{i}$ and $\mathcal{D}$ satisfy Convention 4.7. Then the homotopy functor model structure obtained from the projective model structure on the category $\operatorname{Fun}\left(\mathcal{C}_{1} \times \cdots \times \mathcal{C}_{n}, \mathcal{D}\right)$ exists. Furthermore, it is a right proper cofibrantly generated $\mathcal{S}$-model structure, and it is left proper if $\mathcal{D}$ is left proper.

We want to obtain an hf model structure on the category $\operatorname{Fun}\left(\mathcal{C}_{1} \wedge \cdots \wedge \mathcal{C}_{n}, \mathcal{D}\right)$. Unlike the corresponding cartesian product category, the underlying category of $\mathcal{B}_{1} \wedge \cdots \wedge \mathcal{B}_{n}$ is usually neither cocomplete, nor complete (Definition 2.22 addresses their relation). In particular, we cannot directly apply the results from Section 4.2. We first define homotopy functors.

Definition 4.19 A functor $\underset{\sim}{X}$ in $\operatorname{Fun}\left(\mathcal{C}_{1} \wedge \cdots \wedge \mathcal{C}_{n}, \mathcal{D}\right)$ is called a homotopy functor if for any object $\left(K_{1}, \ldots, \widehat{K}_{i}, \ldots, K_{n}\right)$ in $\mathcal{C}_{1} \wedge \cdots \wedge \widehat{\mathcal{C}}_{i} \wedge \cdots \wedge \mathcal{C}_{n}$ the associated partial functor

$$
\underset{\sim}{X}\left(K_{1}, \ldots, \hat{K}_{i}, \ldots, K_{n}\right): \mathcal{C}_{i} \rightarrow \mathcal{D}
$$

is a homotopy functor. The hat indicates that the corresponding entry is left out.

A coaugmented $\mathcal{S}$-functor Fibr from $\mathcal{B}_{1} \wedge \cdots \wedge \mathcal{B}_{n}$ to itself is defined by

$$
\operatorname{Fibr}(\underline{K}):=\left(\operatorname{Fibr}_{\mathcal{B}_{1}}\left(K_{1}\right), \ldots, \operatorname{Fibr}_{\mathcal{B}_{n}}\left(K_{n}\right)\right),
$$

using the decent fibrant replacement in each category $\mathcal{B}_{i}$. Analogous to (4-1), a coaugmented $\mathcal{S}$-functor

$$
\left({ }_{-}\right)^{\text {hf }}: \operatorname{Fun}\left(\mathcal{C}_{1} \wedge \cdots \wedge \mathcal{C}_{n}, \mathcal{D}\right) \rightarrow \operatorname{Fun}\left(\mathcal{C}_{1} \wedge \cdots \wedge \mathcal{C}_{n}, \mathcal{D}\right)
$$

is defined by

$$
\phi_{\underset{\sim}{X}}: \underset{\sim}{X} \rightarrow(\underset{\sim}{X})^{\mathrm{hf}}(\underline{K}):=\left(\left(i^{\wedge n}\right)_{*} \underset{\sim}{X}\right) \circ \text { Fibr } \circ i^{\wedge n},
$$

where $i: \mathcal{C}_{1} \wedge \cdots \wedge \mathcal{C}_{n} \rightarrow \mathcal{B}_{1} \wedge \cdots \wedge \mathcal{B}_{n}$ is the inclusion. These enriched functors are well defined by Proposition 2.16. 
Definition 4.20 A functor $\underset{\sim}{X}$ in $\operatorname{Fun}\left(\Sigma_{n} \prec \mathcal{C}^{\wedge n}, \mathcal{D}\right)$ is called a homotopy functor if $\varepsilon^{*} \underset{\sim}{X}$ is a homotopy functor, where $\varepsilon: \mathcal{C}^{\wedge n} \rightarrow \Sigma_{n} \prec \mathcal{C}^{\wedge n}$ was defined in Definition 3.5.

We observe that the previous constructions extend to the wreath product category. The inclusion $\mathcal{C} \rightarrow \mathcal{B}$ induces a symmetric inclusion $\Sigma_{n} \prec \mathcal{C}^{\wedge n} \rightarrow \Sigma_{n} \prec \mathcal{B}^{\wedge n}$ and a decent fibrant replacement functor of $\mathcal{B}$ extends to a symmetric functor

$$
\text { Fibr }=\left(\operatorname{Fibr}_{\mathcal{B}}, \ldots, \operatorname{Fibr}_{\mathcal{B}}\right): \Sigma_{n} \curlywedge \mathcal{B}^{\wedge n} \rightarrow \Sigma_{n} \curlywedge \mathcal{B}^{\wedge n}
$$

which is a decent fibrant replacement functor for $\Sigma_{n} 2 \mathcal{B}^{\wedge n}$. There is then a coaugmented $\mathcal{S}$-functor

$$
\left({ }_{-}\right)^{\mathrm{hf}}: \operatorname{Fun}\left(\Sigma_{n} \curlywedge \mathcal{C}^{\wedge n}, \mathcal{D}\right) \rightarrow \operatorname{Fun}\left(\Sigma_{n} \curlywedge \mathcal{C}^{\wedge n}, \mathcal{D}\right)
$$

as above and an associated $\mathcal{S}$-natural transformation $\phi_{X}$. In order to treat functors out of the smash product category and the wreath product category simultaneously, the construction $(-)^{\mathrm{hf}}$ has to be interpreted appropriately in the following statements.

Definition 4.21 A map $f: \underset{\sim}{X} \rightarrow \underset{\sim}{Y}$ in $\operatorname{Fun}\left(\mathcal{C}_{1} \wedge \cdots \wedge \mathcal{C}_{n}, \mathcal{D}\right)$ or $\operatorname{Fun}\left(\Sigma_{n} \prec \mathcal{C}^{\wedge n}, \mathcal{D}\right)$ is called:

(1) An $h f$ equivalence if the map $f^{\mathrm{hf}}: \underset{\sim}{X^{\mathrm{hf}}} \rightarrow \underset{\sim}{Y} \mathrm{hf}$ is an objectwise weak equivalence.

(2) An hf fibration if it is an objectwise fibration such that the square

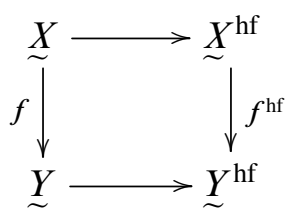

is a homotopy pullback square in the objectwise model structure.

The hf cofibrations are the projective cofibrations.

Remark 4.22 For any $\underset{\sim}{X}$ in $\operatorname{Fun}\left(\mathcal{C}_{1} \wedge \cdots \wedge \mathcal{C}_{n}, \mathcal{D}\right)$ or $\operatorname{Fun}\left(\Sigma_{n} \curlywedge \mathcal{C}^{\wedge n}, \mathcal{D}\right)$ the functor ${\underset{\sim}{X}}^{\mathrm{hf}}$ is a homotopy functor and analogues of Remark 4.13 hold.

Theorem 4.23 Assume Convention 4.7 holds. The classes given in Definition 4.21 constitute a right proper cofibrantly generated $\mathcal{S}$-model structure on the categories $\operatorname{Fun}\left(\mathcal{C}_{1} \wedge \cdots \wedge \mathcal{C}_{n}, \mathcal{D}\right)$ and $\operatorname{Fun}\left(\Sigma_{n} \prec \mathcal{C}^{\wedge n}, \mathcal{D}\right)$, respectively. It is left proper if $\mathcal{D}$ is left proper.

Proof The arguments of the proof of Theorem 4.14 showing the existence of the hf model structure on $\operatorname{Fun}(\mathcal{C}, \mathcal{D})$ apply componentwise. 
The model structure from Theorem 4.23 is called the homotopy functor model structure and is denoted by $\operatorname{Fun}\left(\mathcal{C}_{1} \wedge \cdots \wedge \mathcal{C}_{n}, \mathcal{D}\right)_{\mathrm{hf}}$ and $\operatorname{Fun}\left(\Sigma_{n} \curlywedge \mathcal{C}^{\wedge n}, \mathcal{D}\right)_{\mathrm{hf}}$, respectively.

Proposition 4.24 Assume Convention 4.7. The adjoint pair

$$
p_{*}: \operatorname{Fun}\left(\mathcal{C}_{1} \times \cdots \times \mathcal{C}_{n}, \mathcal{D}\right)_{\mathrm{hf}} \rightleftarrows \operatorname{Fun}\left(\mathcal{C}_{1} \wedge \cdots \wedge \mathcal{C}_{n}, \mathcal{D}\right)_{\mathrm{hf}}: p^{*}
$$

is a Quillen pair of homotopy functor model structures. The functor $p^{*}$ preserves and detects weak equivalences and fibrations. Also, the adjoint pair

$$
\varepsilon_{*}: \operatorname{Fun}\left(\mathcal{C}^{\wedge n}, \mathcal{D}\right)_{\mathrm{hf}} \rightleftarrows \operatorname{Fun}\left(\Sigma_{n} \prec \mathcal{C}^{\wedge n}, \mathcal{D}\right)_{\mathrm{hf}}: \varepsilon^{*}
$$

is a Quillen pair of homotopy functor model structures. The functor $\varepsilon^{*}$ preserves and detects weak equivalences and fibrations.

Proof The pair $\left(p_{*}, p^{*}\right)$ is a Quillen pair for the respective projective model structures by Lemma 2.23. The same holds for the pair $\left(\varepsilon_{*}, \varepsilon^{*}\right)$. The claims above then follow from the canonical natural isomorphisms $p^{*} \circ(-)^{\mathrm{hf}} \cong(-)^{\mathrm{hf}} \circ p^{*}$ and $\varepsilon^{*} \circ(-)^{\mathrm{hf}} \cong$ $(-)^{\mathrm{hf}} \circ \varepsilon^{*}$.

Lemma 4.25 Consider the homotopy functor model structure on $\operatorname{Fun}(\mathcal{C}, \mathcal{D})$ obtained from the cross effect model structure. Then the $n^{\text {th }}$ cross effect

$$
\operatorname{cr}_{n}: \operatorname{Fun}(\mathcal{C}, \mathcal{D})_{\mathrm{hf}-\mathrm{cr}} \rightarrow \operatorname{Fun}\left(\Sigma_{n} \curlywedge \mathcal{C}^{\wedge n}, \mathcal{D}\right)_{\mathrm{hf}}
$$

is a right Quillen functor.

Proof Proposition 3.25 implies that the left adjoint $\mathcal{L}_{n}$ of $\mathrm{cr}_{n}$ preserves cofibrations. In order to prove that $\mathcal{L}_{n}$ also preserves acyclic cofibrations in the homotopy functor model structure, it suffices to prove that $\mathrm{cr}_{n}$ maps fibrant objects to fibrant objects in the respective homotopy functor model structures. To do so, let $\underset{\widetilde{\alpha}}{X}: \mathcal{C} \rightarrow \mathcal{D}$ be a cross effect fibrant homotopy functor. $\left.\operatorname{Then} \mathrm{cr}_{n} \underset{\sim}{X}\right)$ is objectwise weakly equivalent to $\operatorname{hocr}_{n}(\underset{\sim}{X})$ by Lemma 3.24, and the latter is a homotopy functor by construction.

\section{Excisive functors}

The goal of this section is to localize the homotopy functor model structures on the various functor categories further, such that every functor is weakly equivalent to an $n$-excisive functor. Fibrant replacement in such a model structure then serves as $n$-excisive approximation. Recall that a homotopy functor is $n$-excisive if it maps strongly homotopy cocartesian $(n+1)$-cubes to homotopy Cartesian ones. 


\subsection{The excisive model structures}

Set $\underline{n}:=\{1, \ldots, n\}$, let $\mathcal{P}(\underline{n})$ be its power set, and let $\mathcal{P}_{0}(\underline{n}):=\mathcal{P}(\underline{n})-\{\varnothing\}$.

Definition 5.1 For an object $A$ in $\mathcal{C}$, let $C A$ be the simplicial cone over $A$. This is the reduced or unreduced cone depending on whether $\mathcal{C}$ is a $\mathcal{U}-$ or $\mathcal{S}$-category. For a finite set $U$, the join $A \star U$ is defined as

$$
C A \sqcup_{A} \cdots \sqcup_{A} C A,
$$

gluing $|U|$ many copies of $C A$ along their base $A$.

Convention 5.2 In addition to Convention 4.7, suppose that for any object $A$ in $\mathcal{C}$ and any finite set $U$ the object $A \star U$ is also in $\mathcal{C}$.

Remark 5.3 There are other models for $A \star U$. In an ambient model category $\mathcal{B}$, the join $A \star U$ is weakly equivalent to a homotopy colimit of the asterisk-shaped diagram given by $|U|$ copies of the map $A \rightarrow *$ out of a single copy of $A$. For instance, $A \star U$ is weakly equivalent to $|U|-1$ wedge summands of $\Sigma A$. Hence, the assumption that $\mathcal{C}$ is closed under suspensions and finite coproducts is an equally good convention. Because $\mathcal{C}$ is a full subcategory of $\mathcal{B}$, a reasonable sufficient condition is to assume that $\mathcal{C}$ is closed under finite pushouts along cofibrations.

The join is an enriched bifunctor and comes with a natural map $A \rightarrow A \star U$ induced by the inclusion $\varnothing \subset U$. The $\mathcal{P}_{0}(\underline{n+1})$-diagram $U \mapsto R^{U \star A}$ of representable functors

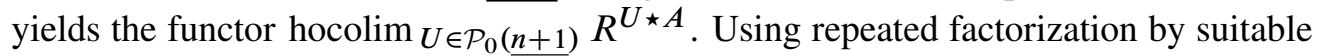
simplicial mapping cylinder constructions supplies a cr cofibrant model, denoted as

$$
\underset{\sim}{A_{n}} \stackrel{\simeq}{\longrightarrow} \operatorname{hocolim}_{U \in \mathcal{P}_{0}(\underline{\underline{n+1}})} R^{U \star A} .
$$

The induced natural transformation $\underset{\sim}{A_{n}} \rightarrow R^{A}$ is factored via a simplicial mapping cylinder as a cr cofibration $\xi_{A, n}$, followed by a simplicial homotopy equivalence:

$$
\underset{\sim}{A_{n}} \stackrel{\xi_{A, n}}{\longrightarrow} \operatorname{Cyl}\left(\xi_{A, n}\right) \stackrel{\simeq}{\rightarrow} R^{A} .
$$

Definition 5.4 Goodwillie's construction $T_{n}$ [11, page 657] on the category of objectwise fibrant homotopy functors may be rewritten as

$$
T_{n} \underset{\sim}{X}(A):=\operatorname{hom}(\underset{\sim}{A}, \underset{\sim}{X}) .
$$

Let $P_{n}$ be the colimit of the following sequence

$$
\mathrm{Id} \rightarrow(-)^{\mathrm{hf}} \rightarrow T_{n}(-)^{\mathrm{hf}} \rightarrow T_{n}^{2}(-)^{\mathrm{hf}} \rightarrow \cdots \rightarrow \underset{k}{\operatorname{colim}} T_{n}^{k}(-)^{\mathrm{hf}} .
$$


Convention 4.7 implies that filtered colimits and filtered homotopy colimits are weakly equivalent in $\mathcal{D}$. Thus, Goodwillie's $n$-excisive approximation is weakly equivalent to $P_{n}$ as defined above. The canonical inclusion $\underline{n} \rightarrow \underline{n+1}$ induces a map $\mathcal{P}_{0}(\underline{n}) \hookrightarrow$ $\mathcal{P}_{0}(\underline{n+1)})$ of posets. This induces natural transformations $T_{n} \rightarrow T_{n-1}$ and $q_{n}: P_{n} \rightarrow$ $P_{n-1}$ which commute with the coaugmentations from the identity. The map $q_{n}$ is constructed for categories of spaces or spectra in [11, page 664] and generalizes to this setup.

Lemma 5.5 The functor $T_{n}$ commutes up to natural weak equivalence with all homotopy limits. The functor $P_{n}$ commutes up to natural weak equivalence with finite homotopy limits. Both functors commute up to natural weak equivalence with filtered homotopy colimits.

Proof Since $\underset{\sim}{A}$ is cr cofibrant, $T_{n}$ is a right Quillen functor and commutes with all homotopy limits. Part (4) of Convention 4.7 ensures that $T_{n}$ commutes with filtered colimits. It then follows from its definition that $P_{n}$ commutes with filtered colimits. Again Convention 4.7 implies that $P_{n}$ commutes at least with finite homotopy limits.

Lemma 5.6 Let $\underset{\sim}{X}$ be an objectwise fibrant homotopy functor.

(1) The functor $P_{n} \underset{\sim}{X}$ is $n$-excisive.

(2) The map $\underset{\sim}{X} \rightarrow P_{n} \underset{\sim}{X}$ is initial among maps in the objectwise homotopy category out of $\underset{\sim}{X}$ into an $n$-excisive functor.

(3) Both maps $P_{n}\left(p_{n} \underset{\sim}{X}\right)$ and $p_{n}\left(P_{n} \underset{\sim}{X}\right)$ are objectwise weak equivalences and homotopic to each other.

Proof Part (1) follows by adapting Goodwillie's opaque [11, page 662] or Rezk's slightly less opaque proof [20]. Parts (2) and (3) are as in [11, page 661].

Definition 5.7 A map $\underset{\sim}{X} \rightarrow \underset{\sim}{Y}$ in $\operatorname{Fun}(\mathcal{C}, \mathcal{D})$ is called:

(1) An $n$-excisive equivalence if the induced map $P_{n} \underset{\sim}{X} \rightarrow P_{n} \underset{\sim}{Y}$ is an objectwise weak equivalence.

(2) An $n$-excisive fibration if it is an hf fibration and the following diagram

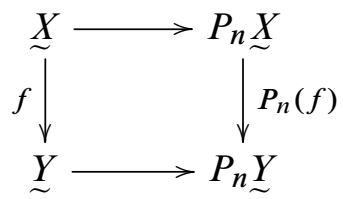

is a homotopy pullback square in the homotopy functor model structure.

The $n$-excisive cofibrations are projective cofibrations. Analogous definitions can be given starting from the cr model structure. 
In the case of $\mathcal{D}=\mathcal{S}$ and $\mathcal{C}=\mathcal{S}^{\text {fin }}$, the following theorem was already obtained in our previous article [3].

Theorem 5.8 Assume Convention 5.2. The classes described in Definition 5.7 form a right proper cofibrantly generated $\mathcal{S}$-model structure on $\operatorname{Fun}(\mathcal{C}, \mathcal{D})$, which is left proper if $\mathcal{D}$ is left proper.

Proof It suffices to show that the coaugmented functor $P_{n}$ satisfies the axioms (A1), (A2), and (A3) given by Bousfield in $[5,9.2]$. The functors $T_{n}$ and $(-)^{\text {hf }}$ preserve objectwise weak equivalences by construction and the proof of Theorem 4.14, respectively. Filtered colimits in $\mathcal{D}$ preserve weak equivalences by part (4) of Convention 4.7, whence $P_{n}$ also preserves objectwise weak equivalences. This implies (A1). Property (A2) is verified in Lemma 5.6(3). Property (A3) follows directly from Lemma 5.5. The remaining task is to add further generating acyclic cofibrations for the $n$-excisive model structure. With $I_{\mathcal{D}}$ being a set of generating cofibrations of $\mathcal{D}$, let

$$
J_{n}:=\left\{\xi_{A, n} \square i\right\}_{A \in \mathcal{C}, i \in I_{\mathcal{D}}} .
$$

An objectwise fibration $\underset{\sim}{X} \rightarrow \underset{\sim}{Y}$ has the right lifting property with respect to the set $J_{n}$ if and only if the morphism of pointed simplicial sets

$$
\left.\mathcal{S}_{\mathcal{D}}\left(\operatorname{Cyl}\left(\xi_{A, n}\right), \underset{\sim}{X}\right) \rightarrow \mathcal{S}_{\mathcal{D}}(\underset{\sim}{A}, \underset{\sim}{X}) \times_{\mathcal{S}_{\mathcal{D}}\left(A_{n}, Y \sim\right.}\right) \mathcal{S}_{\mathcal{D}}\left(\operatorname{Cyl}\left(\xi_{A, n}\right), \underset{\sim}{Y}\right)
$$

has the right lifting property with respect to $I_{\mathcal{D}}$. Since the map in (5-1) is a fibration in $\mathcal{D}$ anyway, $\underset{\sim}{X} \rightarrow \underset{\sim}{Y}$ has the right lifting property with respect to $J_{n}$ if and only if (5-1) is a weak equivalence. The simplicial homotopy equivalence

$$
\operatorname{Cyl}\left(\xi_{A, n}\right) \stackrel{\simeq}{\rightarrow} R^{A}
$$

induces a simplicial homotopy equivalence on $\mathcal{D}$-mapping objects. As $\mathcal{S}_{\mathcal{D}}(-, \underset{\sim}{Z})$ transforms homotopy colimits to homotopy limits for $\underset{\sim}{Z}$ objectwise fibrant, and $\underset{\sim}{X} \rightarrow \underset{\sim}{\underset{\sim}{Y}}$ is an objectwise fibration, the map $\underset{\sim}{X} \rightarrow \underset{\sim}{Y}$ has the right lifting property with respect to $J_{n}$ if and only if the square

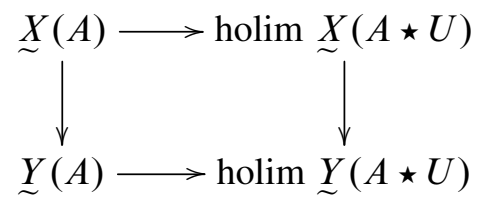

is a homotopy pullback square. Definition 5.4 of $T_{n}$ implies that there is a natural zig-zag of weak equivalences connecting this commutative diagram to the commutative 
diagram

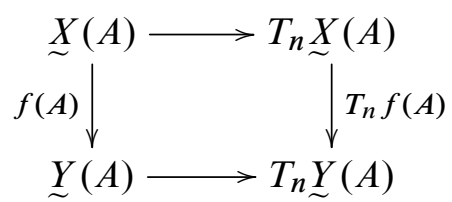

which is a homotopy pullback square by Lemma 5.9. Thus adding $J_{n}$ to a suitable set of generating acyclic cofibrations for the $\mathrm{hf}$ model structure yields a set of generating acyclic cofibrations for $n$-excisive fibrations; analogously for their cr versions.

The model structures provided by Theorem 5.8 are the $n$-excisive model structures. They are denoted $\operatorname{Fun}(\mathcal{C}, \mathcal{D})_{n \text {-exc }}$ and $\operatorname{Fun}(\mathcal{C}, \mathcal{D})_{n \text {-exc-cr }}$, respectively. The following statement is analogous to Lemma 4.15.

Lemma 5.9 A map $f: \underset{\sim}{X} \rightarrow \underset{\sim}{Y}$ is an $n$-excisive fibration if and only if it is an $h f$ fibration and the following diagram

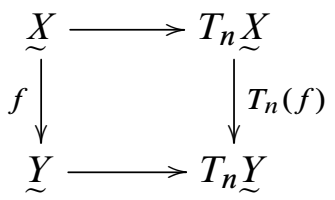

is a homotopy pullback square in the hf model structure.

Proof This is straightforward using the fact that in $\mathcal{D}$ filtered colimits preserve homotopy pullbacks.

Remark 5.10 The proof of Theorem 5.8 shows that the $n$-excisive (cr) model structure is a left Bousfield localization of the $\mathrm{hf}(\mathrm{cr})$ model structure with respect to the set $\left\{\xi_{A, n} \mid A \in \mathcal{C}\right\}$ or equivalently the set

$$
\left\{\underset{U \in \mathcal{P}_{0}(\underline{n+1})}{\operatorname{hocolim}} R^{U \star A} \rightarrow R^{A} \mid A \in \mathcal{C}\right\} .
$$

\subsection{Excisive functors in several variables}

Definition 5.11 A functor $\underset{\sim}{X}: \mathcal{C}_{1} \times \cdots \times \mathcal{C}_{n} \rightarrow \mathcal{D}$ is multi-excisive if for every object $\left(K_{1}, \ldots, \widehat{K}_{i}, \ldots, K_{n}\right)$ in $\mathcal{C}_{1} \times \cdots \times \widehat{\mathcal{C}}_{i} \times \cdots \times \mathcal{C}_{n}$ the associated partial functor

$$
\left.\underset{\sim}{X} K_{1}, \ldots, \hat{K}_{i}, \ldots, K_{n}\right): \mathcal{C}_{i} \rightarrow \mathcal{D}
$$

is excisive. A functor $\underset{\sim}{X}$ is $\left(d_{1}, \ldots, d_{n}\right)$-excisive if, for every $1 \leq i \leq n$, the associated partial functor $\underset{\sim}{X}\left(K_{1}, \ldots, \hat{K}_{i}, \ldots, K_{n}\right)$ is $d_{i}$-excisive. 
The augmented functor $P_{1}$ from Definition 5.4 can be applied componentwise to functors in $\operatorname{Fun}\left(\mathcal{C}_{1} \times \cdots \times \mathcal{C}_{n}, \mathcal{D}\right)$. More precisely, for each object $\underline{K}$ in $\mathcal{C}_{1} \times \cdots \times \mathcal{C}_{n}$ and each $1 \leq i<j \leq n$, the diagram

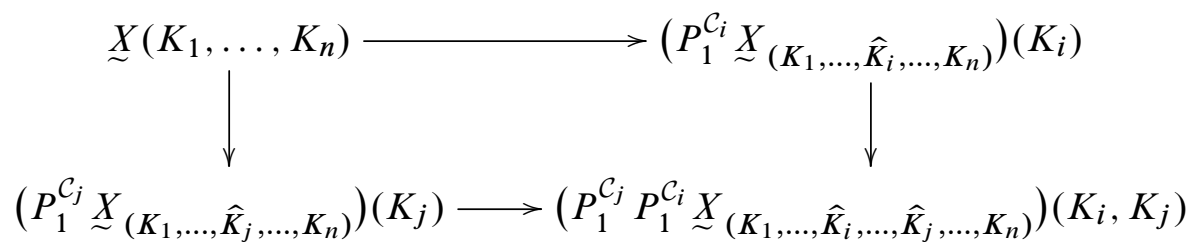

in $\mathcal{D}$ is natural in $\underline{K}$ and $\underset{\sim}{X}$. By definition, $P_{1}^{\mathcal{C}_{i}}$ and $P_{1}^{\mathcal{C}_{j}}$ commute with each other up to canonical isomorphism, and thus the order $i<j$ is purely cosmetic. These diagrams assemble into a $\mathcal{U}$-natural transformation

$$
p_{1, \ldots, 1}(\underset{\sim}{X}): \underset{\sim}{X} \rightarrow P_{1, \ldots, 1} \underset{\sim}{X}:=P_{1}^{\mathcal{C}_{1}} \ldots P_{1}^{\mathcal{C}_{n}} \underset{\sim}{X}
$$

of functors from $\mathcal{C}_{1} \times \cdots \times \mathcal{C}_{n}$ by Propositions 2.15 and 2.16. If the functor $\underset{\sim}{X}$ is multireduced, then this natural transformation is in fact enriched over $\mathcal{S}$.

A functor $\underset{\sim}{X}$ is multi-excisive if and only if the natural transformation $p_{1, \ldots, 1}(\underset{\sim}{X})$ is an objectwise weak equivalence.

Definition 5.12 A map $f: \underset{\sim}{X} \rightarrow \underset{\sim}{Y}$ in $\operatorname{Fun}\left(\mathcal{C}_{1} \times \cdots \times \mathcal{C}_{n}, \mathcal{D}\right)$ is:

(1) A multi-excisive equivalence if the map $P_{1, \ldots, 1}(f)$ is an hf equivalence.

(2) A multi-excisive fibration if it is an hf fibration such that the square

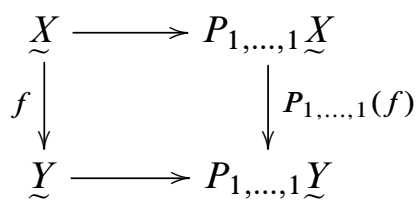

is a homotopy pullback square in the hf model structure.

The multi-excisive cofibrations are the projective cofibrations.

Theorem 5.13 The classes given in Definition 5.12 are a right proper cofibrantly generated $\mathcal{S}$-model structure on $\operatorname{Fun}\left(\mathcal{C}_{1} \times \cdots \times \mathcal{C}_{n}, \mathcal{D}\right)$, which is left proper if $\mathcal{D}$ is left proper.

Proof The fact that each functor $P_{1}^{\mathcal{C}_{i}}$ above satisfies properties (A1), (A2) and (A3) by the proof of Theorem 5.8 implies that the composite functor $P_{1, \ldots, 1}$ satisfies them. Generating acyclic cofibrations are constructed as in the proof of Theorem 5.8. 
The corresponding model structure on $\operatorname{Fun}\left(\mathcal{C}_{1} \times \cdots \times \mathcal{C}_{n}, \mathcal{D}\right)$ is called the multi-excisive model structure and is denoted $\operatorname{Fun}\left(\mathcal{C}_{1} \times \cdots \times \mathcal{C}_{n}, \mathcal{D}\right)_{\text {mltexc }}$.

\subsection{Multilinear and symmetric multilinear functors}

The natural transformation $p_{1, \ldots, 1}$ from (5-2) for functors $\underset{\sim}{X}: \mathcal{C}_{1} \times \cdots \times \mathcal{C}_{n} \rightarrow \mathcal{D}$ will be constructed differently for functors in $\operatorname{Fun}\left(\mathcal{C}_{1} \wedge \cdots \wedge \widetilde{\mathcal{C}}_{n}, \mathcal{D}\right)$ and $\Sigma_{n} \prec \mathcal{C}^{\wedge n}$. Let $S^{n}$ denote the $n$-fold smash product of $S^{1}=\Delta^{1} / \partial \Delta^{1}$. Adapting Convention 5.2 slightly, if necessary, each of the categories $\mathcal{C}_{k}$ is closed under tensoring with $S^{1}$ as a sub-S -category of the ambient model category $\mathcal{B}_{k}$. In particular, every object $\underline{K}$ has a functorial suspension $\underline{K} \wedge S^{1}=\left(K_{1} \wedge S^{1}, \ldots, K_{n} \wedge S^{1}\right)$, allowing the following observation.

Definition 5.14 For every object $\underline{K}$ in $\mathcal{C}_{1} \wedge \cdots \wedge \mathcal{C}_{n}$ there is a natural map

$$
\begin{aligned}
\mathcal{S}_{\mathcal{C}_{1} \wedge \cdots \wedge \mathcal{C}_{n}}\left(\underline{K} \wedge S^{1}, \underline{K} \wedge S^{1}\right) & \left.=\bigwedge_{i=1}^{n} \mathcal{S}_{\mathcal{C}_{i}}\left(K_{i} \wedge S^{1}, K_{i} \wedge S^{1}\right)\right) \\
& \cong \bigwedge_{i=1}^{n} \mathcal{S}\left(S^{1}, \mathcal{S}_{\mathcal{C}_{i}}\left(K_{i}, K_{i} \wedge S^{1}\right)\right) \\
& \stackrel{f}{\rightarrow} \mathcal{S}\left(S^{n}, \bigwedge_{i=1}^{n} \mathcal{S}_{\mathcal{C}_{i}}\left(K_{i}, K_{i} \wedge S^{1}\right)\right) \\
& =\mathcal{S}\left(S^{n}, \mathcal{S}_{\mathcal{C}_{1} \wedge \cdots \wedge \mathcal{C}_{n}}\left(\underline{K}, \underline{K} \wedge S^{1}\right)\right) .
\end{aligned}
$$

The isomorphism uses that the ambient categories $\mathcal{B}_{i}$ are tensored over $\mathcal{S}$. The map labeled $f$ is an $n$-fold version of the canonical map

$$
\mathcal{S}\left(S^{1}, L\right) \wedge \mathcal{S}\left(S^{1}, M\right) \rightarrow \mathcal{S}\left(S^{1} \wedge S^{1}, L \wedge M\right) .
$$

If $\underset{\sim}{X}$ is a functor in $\operatorname{Fun}\left(\mathcal{C}_{1} \wedge \cdots \wedge \mathcal{C}_{n}, \mathcal{D}\right)$, the composition above induces a map

$$
S^{n} \rightarrow \mathcal{S}_{\mathcal{C}_{1} \wedge \cdots \wedge \mathcal{C}_{n}}\left(\underline{K}, \underline{K} \wedge S^{1}\right) \rightarrow \mathcal{S}_{\mathcal{D}}\left(\underset{\sim}{X}(\underline{K}), \underset{\sim}{X}\left(\underline{K} \wedge S^{1}\right)\right) .
$$

that has an adjoint map

$$
\underset{\sim}{X}(\underline{K}) \rightarrow\left(\underset{\sim}{X}\left(\underline{K} \wedge S^{1}\right)\right)^{S^{n}}
$$

which is natural in $\underline{K}$. The result is a composite natural transformation

$$
t_{\sim}^{X}: \underset{\sim}{X} \rightarrow T_{(1, \ldots, 1)}(\underset{\sim}{X}):=\Omega^{n}\left(\underset{\sim}{X}\left(\_\wedge S^{1}\right)\right)
$$


of $\mathcal{S}$-functors. Let $\left.p_{(1, \ldots, 1)}(\underset{\sim}{X}): \underset{\sim}{X} \rightarrow P_{(1, \ldots, 1)} \underset{\sim}{X}\right)$ denote the canonical map to the colimit

$$
\underset{\sim}{X} \rightarrow \underset{\sim}{X} \mathrm{hf} \stackrel{t_{X} \mathrm{hf}}{\longrightarrow} T_{(1, \ldots, 1)}\left(\underset{\sim}{X}{ }^{\mathrm{hf}}\right) \stackrel{t_{T_{(1, \ldots, 1)}\left(X^{\mathrm{hf}}\right)}^{\longrightarrow}}{\longrightarrow} \cdots \rightarrow \operatorname{colim}_{n} T_{(1, \ldots, 1)}^{n}\left(\underset{\sim}{X^{\mathrm{hf}}}\right) .
$$

One can check that $\left.p^{*}\left(p_{(1, \ldots, 1)} \underset{\sim}{X}\right)\right)$ is canonically weakly equivalent to the natural transformation obtained in (5-2). Here, $p$ is the functor from Definition 2.22.

Remark 5.15 In order to repeat this for symmetric functors, observe that the $n$-fold smash product $S^{n}$ of the unit circle carries a natural $\Sigma_{n}$-action given by permutation.

Definition 5.16 For every object $\underline{K}$ in $\Sigma_{n} \prec \mathcal{C}^{\wedge n}$ there is a natural map

$$
\begin{aligned}
\mathcal{S}_{\Sigma_{n} \mathcal{C}^{\wedge n}}\left(\underline{K} \wedge S^{1}, \underline{K} \wedge S^{1}\right) & \cong \bigvee_{\sigma} \bigwedge_{i=1}^{n} \mathcal{S}\left(S^{1}, \mathcal{S}_{\mathcal{C}}\left(K_{i}, K_{\sigma^{-1}(i)} \wedge S^{1}\right)\right) \\
& \stackrel{f}{\rightarrow} \bigvee_{\sigma} \mathcal{S}\left(S^{n}, \bigwedge_{i=1}^{n} \mathcal{S}_{\mathcal{C}}\left(K_{i}, K_{\sigma^{-1}(i)} \wedge S^{1}\right)\right) \\
& \stackrel{g}{\rightarrow} \mathcal{S}\left(S^{n}, \mathcal{S}_{\Sigma_{n} \mathcal{C}} \wedge n\left(\underline{K}, \underline{K} \wedge S^{1}\right)\right),
\end{aligned}
$$

where the first two maps are as in Definition 5.14, and the map $g$ is the natural map relating the compositions of coproduct and the functor $\mathcal{S}\left(S^{n},-\right)$. The induced map

$$
S^{n} \rightarrow \mathcal{S}_{\Sigma_{n} \mathcal{C}^{\wedge n}}\left(\underline{K}, \underline{K} \wedge S^{1}\right) \rightarrow \mathcal{S}_{\mathcal{D}}\left(\underset{\sim}{X}(\underline{K}), \underset{\sim}{X}\left(\underline{K} \wedge S^{1}\right)\right)
$$

for any functor $\underset{\sim}{X}$ in $\operatorname{Fun}\left(\Sigma_{n} \prec \mathcal{C}^{\wedge n}, \mathcal{D}\right)$ has as adjoint the map

$$
\underset{\sim}{X}(\underline{K}) \rightarrow\left(\underset{\sim}{X}\left(\underline{K} \wedge S^{1}\right)\right)^{S^{n}},
$$

where the target has the correct $\Sigma_{n}$-action from Definition 3.7 and Remark 5.15. The resulting map of symmetric $\mathcal{S}$-functors is denoted

$$
t_{\underset{\sim}{X}}: \underset{\sim}{X} \rightarrow T_{(1, \ldots, 1)}(\underset{\sim}{X})=\left(\underset{\sim}{X}\left(\_\wedge S^{1}\right)\right)^{S^{n}} .
$$

Let $\left.p_{\sim}^{X}: \underset{\sim}{X} \rightarrow P_{(1, \ldots, 1)} \underset{\sim}{X}\right)$ denote the canonical map to the colimit of the sequence

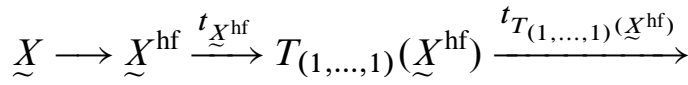

$$
\begin{aligned}
& \cdots \longrightarrow \operatorname{colim} T_{n}^{n}\left(\underset{\sim}{X}{ }^{\mathrm{hf}}\right)=: P_{(1, \ldots, 1)} \underset{\sim}{X} .
\end{aligned}
$$

Definition 5.17 A functor $\underset{\sim}{X}: \mathcal{C}_{1} \wedge \cdots \wedge \mathcal{C}_{n} \rightarrow \mathcal{D}$ is called multilinear if it is multiexcisive in the sense of Definition 5.11, that is, if all partial functors are excisive. A functor $\underset{\sim}{X}: \Sigma_{n} \prec \mathcal{C}^{\wedge n} \rightarrow \mathcal{D}$ is called multilinear if $\varepsilon^{*} \underset{\sim}{X}: \mathcal{C}^{\wedge n} \rightarrow \mathcal{D}$ is multilinear. 
In order to treat the categories $\operatorname{Fun}\left(\mathcal{C}_{1} \wedge \cdots \wedge \mathcal{C}_{n}, \mathcal{D}\right)$ and $\operatorname{Fun}\left(\Sigma_{n} \curlywedge \mathcal{C}^{\wedge n}, \mathcal{D}\right)$ simultaneously, the notation in what follows has to be interpreted appropriately.

Remark 5.18 Let $\underset{\sim}{X}$ be a functor in $\operatorname{Fun}\left(\mathcal{C}_{1} \wedge \cdots \wedge \mathcal{C}_{n}, \mathcal{D}\right)$ or $\operatorname{Fun}\left(\Sigma_{n} \curlywedge \mathcal{C}^{\wedge n}, \mathcal{D}\right)$. In particular, $\underset{\sim}{X}$ is multireduced. Hence, being multi-excisive is equivalent to being multilinear. In any case, the functor $P_{(1, \ldots, 1)} \underset{\sim}{X}$ is componentwise excisive by construction and the map $\underset{\sim}{X} \rightarrow P_{(1, \ldots, 1)} \underset{\sim}{X}$ is initial among maps to multilinear functors in the objectwise homotopy category.

Definition 5.19 A map $f: \underset{\sim}{X} \rightarrow \underset{\sim}{Y}$ in $\operatorname{Fun}\left(\mathcal{C}_{1} \wedge \cdots \wedge \mathcal{C}_{n}, \mathcal{D}\right)$ or $\operatorname{Fun}\left(\Sigma_{n} \curlywedge \mathcal{C}^{\wedge n}, \mathcal{D}\right)$ is:

(1) A multilinear equivalence if the map $P_{1, \ldots, 1}(f)$ is an hf equivalence.

(2) A multilinear fibration if it is an hf fibration such that the square

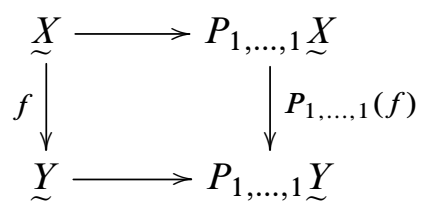

is a homotopy pullback square in the hf model structure.

The multilinear cofibrations are the projective ones.

Theorem 5.20 Assume Convention 4.7. The classes described in Definition 5.19 constitute right proper cofibrantly generated $\mathcal{S}$-model structures on $\operatorname{Fun}\left(\Sigma_{n} \imath \mathcal{C}^{\wedge n}, \mathcal{D}\right)$ and Fun $\left(\mathcal{C}_{1} \wedge \cdots \wedge \mathcal{C}_{n}, \mathcal{D}\right)$, respectively. They are left proper if $\mathcal{D}$ is left proper. The adjoint pairs

$$
p_{*}: \operatorname{Fun}\left(\mathcal{C}_{1} \times \cdots \times \mathcal{C}_{n}, \mathcal{D}\right)_{\mathrm{mlt} \text {-exc }} \rightleftarrows \operatorname{Fun}\left(\mathcal{C}_{1} \wedge \cdots \wedge \mathcal{C}_{n}, \mathcal{D}\right)_{\mathrm{ml}}: p^{*}
$$

and

$$
\varepsilon_{*}: \operatorname{Fun}\left(\mathcal{C}^{\wedge n}, \mathcal{D}\right)_{\mathrm{mlt}-\mathrm{exc}} \rightleftarrows \operatorname{Fun}\left(\Sigma_{n} \curlywedge \mathcal{C}^{\wedge n}, \mathcal{D}\right)_{\mathrm{ml}}: \varepsilon^{*}
$$

are Quillen pairs. The functors $p^{*}$ and $\varepsilon^{*}$ preserve and detect multilinear equivalences and multilinear fibrations.

Proof The assertions about $p^{*}$ and $\varepsilon^{*}$ are straightforward observations. Further, it is tedious but true that $\varepsilon^{*}$ maps the symmetric version (5-6) of the natural transformation $p_{(1, \ldots, 1)}$ to the non-symmetric version (5-4), and that $p^{*}$ maps the $\mathcal{S}$-enriched version (5-4) to the unpointed version (5-2). The proof then proceeds as the proof of Theorem 5.8. In order to describe additional generating acyclic cofibrations, let $\underline{K}$ be 
an object in $\mathcal{C}$. By Lemma 5.21 below, the map $f$ is a multilinear fibration if and only if the diagram

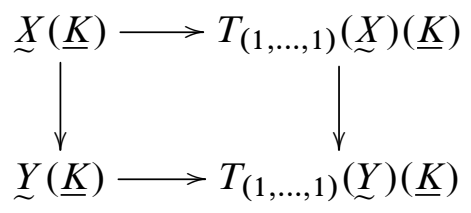

is a homotopy pullback diagram in $\mathcal{D}$ for all $\underline{K}$. The horizontal maps in this square are from (5-3). Evaluated at $\underline{K}$, the upper horizontal map may be written as

$$
\operatorname{hom}\left(R^{\underline{K}}, \underset{\sim}{X}\right) \cong \underset{\sim}{X}(\underline{K}) \rightarrow\left(\underset{\sim}{X}\left(\underline{K} \wedge S^{1}\right)\right)^{S^{n}} \cong \operatorname{hom}\left(R^{\underline{K} \wedge} S^{1} \wedge S^{n}, \underset{\sim}{X}\right) .
$$

By the $\mathcal{S}$-Yoneda Lemma 2.7, it is thus induced by a map

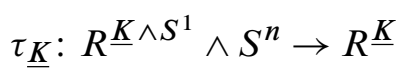

of simplicial functors. Hence, the square above is isomorphic to the following square:

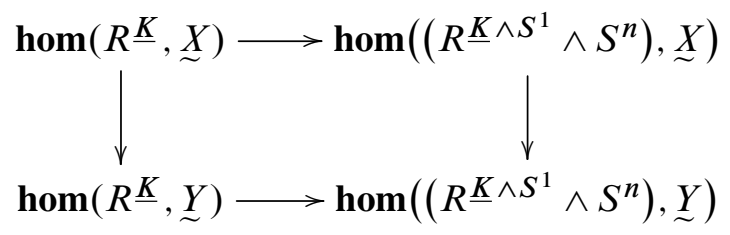

Since $\tau_{\underline{K}}$ is a map between projectively cofibrant objects, the simplicial mapping cylinder yields a factorization as

$$
R^{\underline{K} \wedge} S^{1} \wedge S^{n} \stackrel{j(\underline{K})}{\longrightarrow} \operatorname{Cyl}\left(\tau_{\underline{K}}\right) \stackrel{q(\underline{K})}{\longrightarrow} R \underline{K},
$$

where $j(\underline{K})$ is a projective cofibration, $q(\underline{K})$ is a simplicial homotopy equivalence, and all objects in this factorization are finitely presentable and projectively cofibrant. The square above factors accordingly as follows:

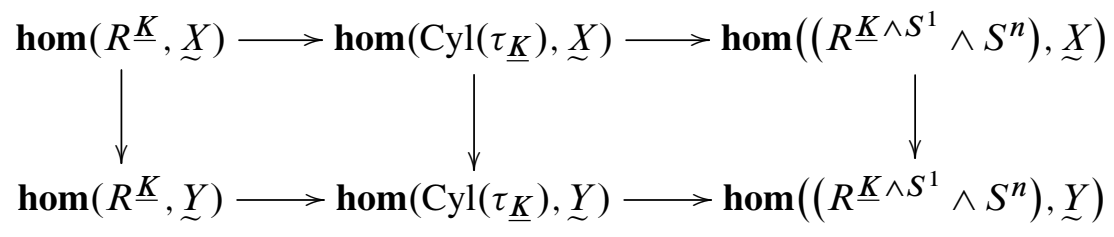

Since the map $q(\underline{K})$ is a simplicial homotopy equivalence, then so are the horizontal maps on the left-hand square. Since the map $j(\underline{K})$ is a projective cofibration and $f$ is 
at least an objectwise fibration, the map

$$
\begin{aligned}
\operatorname{hom}\left(\operatorname{Cyl}\left(\tau_{\underline{K}}\right), \underset{\sim}{X}\right) & \\
& \longrightarrow \operatorname{hom}\left(\operatorname{Cyl}\left(\tau_{\underline{K}}\right), \underset{\sim}{Y}\right) \underset{\operatorname{hom}\left(\left(R^{\underline{K}} \wedge S^{1} \wedge S^{n}\right), \underline{\sim}\right)}{\times} \operatorname{hom}\left(\left(R^{\underline{K} \wedge} S^{1} \wedge S^{n}\right), \underset{\sim}{X}\right)
\end{aligned}
$$

is a fibration in $\mathcal{D}$. Hence, this fibration is acyclic if and only if the square above is a homotopy pullback square. Because $\mathcal{D}$ is cofibrantly generated, the map in question is an acyclic fibration if and only if it has the right lifting property with respect to $I_{\mathcal{D}}$. By adjointness, the map (5-8) is thus a weak equivalence if and only if $f$ has the right lifting property with respect to the set of maps $\{i \square j(\underline{K})\}_{i \in i_{\mathcal{D}}}$.

The model structures provided by Theorem 5.20 are referred to as the multilinear model structures, and denoted $\operatorname{Fun}\left(\mathcal{C}_{1} \wedge \cdots \wedge \mathcal{C}_{n}, \mathcal{D}\right)_{\mathrm{ml}}$ and $\operatorname{Fun}\left(\Sigma_{n} \curlywedge \mathcal{C}^{\wedge n}, \mathcal{D}\right)_{\mathrm{ml}}$, respectively. The proof of Theorem 5.20 shows that they can be seen as left Bousfield localizations.

Lemma 5.21 A map $f: \underset{\sim}{X} \rightarrow \underset{\sim}{Y}$ is a multilinear fibration if and only if it is an hf fibration and the diagram

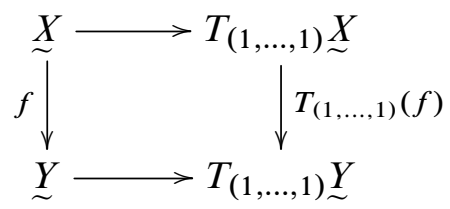

is an objectwise homotopy pullback square.

Proof This is straightforward using the fact that in $\mathcal{D}$ filtered colimits preserve homotopy pullbacks.

\subsection{Coefficient spectra}

The aim of this section is to connect the multilinear model category of symmetric functors with the model category of spectra with an action of a symmetric group.

Definition 5.22 Let $\operatorname{Sp}(\mathcal{D})$ denote the category of Bousfield-Friedlander spectra in the $\mathcal{S}$-model category $\mathcal{D}$ as defined by $\operatorname{Schwede}$ [21]. An object in $\operatorname{Sp}(\mathcal{D})$ is a sequence $\left(E_{0}, E_{1}, \ldots\right)$ of objects in $\mathcal{D}$, together with structure maps $\sigma_{n}^{E}: \Sigma E_{n} \rightarrow E_{n+1}$. A morphism of such objects is a sequence of morphisms in $\mathcal{D}$ commuting strictly with the structure maps. A map $f: E \rightarrow F$ in $\operatorname{Sp}(\mathcal{D})$ is called:

(1) A stable equivalence of spectra in $\mathcal{D}$ if $Q E \rightarrow Q F$ is a levelwise equivalence where $Q$ is a certain model for " $\Omega$-spectrum" given in [21, page 90]. 
(2) A projective cofibration if the map $E_{0} \rightarrow F_{0}$ and for all $n \geq 1$ the maps

$$
E_{n} \vee E_{n-1} F_{n-1} \rightarrow F_{n}
$$

are cofibrations in $\mathcal{D}$.

Theorem 5.23 (Schwede) Suppose $\mathcal{D}$ satisfies parts (3) and (4) of Convention 4.7. Then there is an $\mathcal{S}$-model structure on $\operatorname{Sp}(\mathcal{D})$ with stable equivalences as weak equivalences and projective cofibrations as cofibrations. It satisfies itself Convention 4.7. The $\mathcal{S}$-functor $\mathrm{Ev}_{0}: \operatorname{Sp}(\mathcal{D}) \rightarrow \mathcal{D}, E \mapsto E_{0}$ is a right Quillen functor.

The proof can be found in [21], with the modification that the assumption on properness appearing in [21, Proposition 2.1.5] may be relaxed to right properness by the work of Bousfield [5]. The following ingredients of the proof are relevant later: If $\{i\}_{i \in I_{\mathcal{D}}}$ is a set of generating cofibrations for $\mathcal{D}$, then $\left\{\operatorname{Fr}_{k}(i)\right\}_{k \in \mathbb{N}, i \in I_{\mathcal{D}}}$ is a set of generating cofibrations for $\operatorname{Sp}(\mathcal{D})$. Here the functor $\operatorname{Fr}_{k}: \mathcal{D} \rightarrow \operatorname{Sp}(\mathcal{D})$ is left adjoint to evaluating at the $k^{\text {th }}$ level, explicitly

$$
\left(\operatorname{Fr}_{k}(D)\right)_{\ell}= \begin{cases}* & \text { for } 0 \leq \ell<k \\ \Sigma^{\ell-k} D & \text { for } \ell \geq k\end{cases}
$$

The functor $\mathrm{Ev}_{0}$ commutes with all limits and colimits. It is worth mentioning that $\operatorname{Fun}(\mathcal{C}, \operatorname{Sp}(\mathcal{D}))$ is canonically isomorphic to $\operatorname{Sp}(\operatorname{Fun}(\mathcal{C}, \mathcal{D}))$.

Theorem 5.24 Composing with $\mathrm{Fr}_{0}$ and $\mathrm{Ev}_{0}$ induces a Quillen equivalence

$$
F: \operatorname{Fun}\left(\Sigma_{n} \prec \mathcal{C}^{n}, \mathcal{D}\right)_{\mathrm{ml}} \rightleftarrows \operatorname{Fun}\left(\Sigma_{n} \prec \mathcal{C}^{n}, \operatorname{Sp}(\mathcal{D})\right)_{\mathrm{ml}}: G .
$$

Proof Since the functor $\operatorname{Ev}_{0}: \operatorname{Sp}(\mathcal{D}) \rightarrow \mathcal{D}$ preserves objectwise fibrations and objectwise acyclic fibrations, the same is true for $G$. Hence, $G$ is a right Quillen functor for the projective model structures. The functor $\mathrm{Ev}_{0}$ commutes with all limits, colimits, and is a right Quillen functor. Hence $\operatorname{Ev}_{0}: \operatorname{Sp}(\mathcal{D}) \rightarrow \mathcal{D}$ commutes up to natural weak equivalence with $\underset{\sim}{X} \rightarrow \underset{\sim}{X}$ hf and $T_{(1, \ldots, 1)}$. In particular, the induced functor $G$ is a right Quillen functor on the homotopy functor and the multilinear model structures. A right Quillen functor is a Quillen equivalence if and only if its total right derived functor is an equivalence. The proof of Goodwillie [11, Proposition 3.7], which states that $\mathrm{Ev}_{0}$ induces an equivalence on the (naive) homotopy categories of multilinear functors, extends to the setup here, which concludes the proof.

Corollary 5.25 The multilinear model structure on symmetric functors is stable. 
A suitable evaluation functor connects symmetric functors directly with spectra having a symmetric group action. In order to describe it, recall from Notation 2.2 that the category $\operatorname{Sp}(\mathcal{D})^{\Sigma_{n}}$ is the category of functors $\Sigma_{n} \rightarrow \operatorname{Sp}(\mathcal{D})$, where $\Sigma_{n}$ is viewed as a category with one object. In other words, an object in $\operatorname{Sp}(\mathcal{D})^{\Sigma_{n}}$ is a spectrum with a right $\Sigma_{n}$-action. Since the stable model structure on $\operatorname{Sp}(\mathcal{D})$ is cofibrantly generated, the category $\operatorname{Sp}(\mathcal{D})^{\Sigma_{n}}$ carries a cofibrantly generated model structure with fibrations and weak equivalences defined on underlying spectra. This is sometimes called the model structure for "naive $\Sigma_{n}$-spectra".

Definition 5.26 Precomposition with the symmetric diagonal $\mathcal{S}$-functor

$$
\Delta_{n}: \Sigma_{n} \times \mathcal{C} \rightarrow \Sigma_{n} \prec \mathcal{C}^{\wedge n}
$$

as introduced in the proof of Lemma 3.15, defines a functor

$$
\Delta_{n}^{*}: \operatorname{Fun}\left(\Sigma_{n} \prec \mathcal{C}^{\wedge n}, \operatorname{Sp}(\mathcal{D})\right) \rightarrow \operatorname{Fun}\left(\Sigma_{n} \times \mathcal{C}, \operatorname{Sp}(\mathcal{D})\right) \cong \operatorname{Fun}\left(\mathcal{C}, \operatorname{Sp}(\mathcal{D})^{\Sigma_{n}}\right) .
$$

Evaluating at an object $C$ in $\mathcal{C}$ induces the functor

$$
\operatorname{ev}_{C}: \operatorname{Fun}\left(\mathcal{C}, \operatorname{Sp}(\mathcal{D})^{\Sigma_{n}}\right) \rightarrow \operatorname{Sp}(\mathcal{D})^{\Sigma_{n}}
$$

The composition is denoted

$$
\operatorname{Ev}_{C}=\operatorname{ev}_{C} \circ \Delta_{n}^{*}: \operatorname{Fun}\left(\Sigma_{n} \curlywedge \mathcal{C}^{\wedge n}, \operatorname{Sp}(\mathcal{D})\right) \rightarrow \operatorname{Sp}(\mathcal{D})^{\Sigma_{n}} .
$$

As a composition of two right adjoint functors, the functor $\mathrm{Ev}_{C}$ has a left $\mathcal{S}$-adjoint denoted by

$$
\mathcal{L E v}_{C}: \operatorname{Sp}(\mathcal{D})^{\Sigma_{n}} \rightarrow \operatorname{Fun}\left(\Sigma_{n} \curlywedge(\mathcal{C})^{\wedge n}, \operatorname{Sp}(\mathcal{D})\right)
$$

Theorem 5.27 Suppose that $\mathcal{C}$ is the category $\mathcal{S}^{\text {fin }}$ of finite pointed simplicial sets. The functor

$$
\mathcal{L E v}_{S^{0}}: \operatorname{Sp}(\mathcal{D})^{\Sigma_{n}} \rightarrow \operatorname{Fun}\left(\Sigma_{n} \curlywedge\left(\mathcal{S}^{\mathrm{fin}}\right)^{\wedge n}, \operatorname{Sp}(\mathcal{D})\right)_{\mathrm{ml}}
$$

is a left Quillen equivalence.

Proof Choosing $S^{0} \in \mathcal{C}=\mathcal{S}^{\text {fin }}$ yields the functor

$$
\operatorname{Ev}_{\underline{S}^{0}}: \operatorname{Fun}\left(\Sigma_{n} \prec\left(\mathcal{S}^{\mathrm{fin}}\right)^{\wedge n}, \operatorname{Sp}(\mathcal{D})\right) \rightarrow \operatorname{Sp}(\mathcal{D})^{\Sigma_{n}}
$$

Explicitly, it is given by $\underset{\sim}{X} \mapsto \underset{\sim}{X}\left(S^{0}, \ldots, S^{0}\right)$ with $\Sigma_{n}$-action induced by permuting the $n$-tuple $\left(S^{0}, \ldots, \widetilde{S^{0}}\right)$. Its left adjoint $\mathcal{L E v}_{\underline{S}^{0}}$ sends a $\Sigma_{n}$-spectrum $E$ to the symmetric functor

$$
\underline{K}=\left(K_{1}, \ldots, K_{n}\right) \longmapsto \mathcal{L E v}_{\underline{S}^{0}}(\underline{K})=E \wedge K_{1} \wedge \cdots \wedge K_{n}
$$


having the following effect on morphism spaces:

$$
\begin{aligned}
& \bigvee_{\sigma \in \Sigma_{n}} \bigwedge_{i=1}^{n} \mathcal{S}\left(K_{i}, L_{\sigma^{-1}(i)}\right) \longrightarrow \mathcal{S}\left(E \wedge K_{1} \wedge \cdots \wedge K_{n}, E \wedge L_{1} \wedge \cdots \wedge L_{n}\right), \\
& \quad\left(\sigma, \underline{f}=\left(f_{1}, \ldots, f_{n}\right)\right) \longmapsto \sigma_{E} \wedge\left(\sigma_{*} \circ\left(f_{1} \wedge \cdots \wedge f_{n}\right)\right) .
\end{aligned}
$$

Here $\sigma_{*}$ is the permutation $L_{\sigma^{-1}(1)} \wedge \cdots \wedge L_{\sigma^{-1}(n)} \rightarrow L_{1} \wedge \cdots \wedge L_{n}$ induced by $\sigma$. The unit $E \rightarrow \operatorname{Ev}_{S^{0}}\left(\mathcal{L E v}_{S^{0}}(E)\right)$ is the canonical isomorphism identifying $E$ with $E \wedge S^{0} \wedge \cdots \wedge S^{0}$. The counit $\mathcal{L} \operatorname{Ev}_{S^{0}}\left(\operatorname{Ev}_{S^{0}}(\underset{\sim}{X})\right) \rightarrow \underset{\sim}{X}$ is the natural transformation

$$
\underset{\sim}{X}\left(S^{0}, \ldots, S^{0}\right) \wedge K_{1} \wedge \cdots \wedge K_{n} \rightarrow \underset{\sim}{X}\left(K_{1}, \ldots, K_{n}\right)
$$

which is a special case of the assembly map

$$
\underset{\sim}{X}\left(L_{1}, \ldots, L_{n}\right) \wedge K_{1} \wedge \cdots \wedge K_{n} \rightarrow \underset{\sim}{X}\left(\left(K_{1} \wedge L_{1}\right), \ldots,\left(K_{n} \wedge L_{n}\right)\right) .
$$

The latter is adjoint to the natural map

$$
\begin{gathered}
K_{1} \wedge \cdots \wedge K_{n} \\
\left\{\operatorname{idj} \times \mathcal{S}\left(L_{1}, K_{1} \wedge L_{1}\right) \wedge \cdots \wedge \mathcal{S}\left(L_{n}, K_{n} \wedge L_{n}\right)\right. \\
\Sigma_{n} \curlywedge\left(\mathcal{S}^{\text {fin }}\right)^{\wedge n}\left(\left(L_{1}, \ldots, L_{n}\right),\left(K_{1} \wedge L_{1}, \ldots, K_{n} \wedge L_{n}\right)\right) \\
\downarrow \\
\operatorname{Sp}\left(\underset{\sim}{X}(\underline{L}), \underset{\sim}{X}\left(K_{1} \wedge L_{1}, \ldots, K_{n} \wedge L_{n}\right)\right) .
\end{gathered}
$$

Since $\Delta^{*}$ and $\mathrm{ev}_{S^{0}}$ are right Quillen functors for projective model structures on functor categories, so is their composition. Hence, $\mathcal{L} \mathrm{Ev}_{S^{0}}$ is a left Quillen functor to the projective model structure, and to the multilinear model structure as well.

To show that the derived unit $E \rightarrow \operatorname{Ev}_{S^{0}}\left(P_{(1, \ldots, 1)}\left(\mathcal{L E v}_{S^{0}}(E)\right)\right)$ is a weak equivalence for $E$ cofibrant, recall that the unit is an isomorphism. Further, the functor $\mathcal{L E v}_{S^{0}}(E)$ preserves weak equivalences and the canonical map

$$
E \wedge S^{k} \wedge \cdots \wedge S^{k} \rightarrow \Omega^{n}\left(\left(E \wedge S^{k+1} \wedge \cdots \wedge S^{k+1}\right)^{\mathrm{fib}}\right)
$$

is a weak equivalence in the stable model structure, where $\left(_{-}\right)^{\text {fib }}$ denotes fibrant replacement in $\operatorname{Sp}^{\Sigma_{n}}$. It follows that $E \rightarrow P_{(1, \ldots, 1)}\left(\mathcal{L E v}_{S^{0}}(E)\right)\left(\underline{S}^{0}\right)$ is a weak equivalence. It remains to prove that $\mathrm{Ev}_{S^{0}}$ detects weak equivalences of multilinear functors. As in the proof of Goodwillie [11, Proposition 5.8], the symmetry is irrelevant, and the 
case $n=1$ is sufficient. If $f: \underset{\sim}{X} \rightarrow \underset{\sim}{Y}$ is a map of linear functors with $f\left(S^{0}\right)$ a weak equivalence, then $f\left(S^{k}\right)$ is a weak equivalence for every $k$, as one deduces from the natural weak equivalence (5-3). It then follows that $f(K)$ is a weak equivalence for every $K \in \mathcal{S}^{\text {fin }}$ by induction on the cells in $K$, using that $\underset{\sim}{X}$ and $\underset{\sim}{Y}$ are linear.

For $\underset{\sim}{X}: \Sigma_{n} \imath\left(\mathcal{S}^{\mathrm{fin}}\right)^{\wedge n} \rightarrow \operatorname{Sp}(\mathcal{D})$, the $\Sigma_{n}$-spectrum $\operatorname{Ev}_{S^{0}}(\underset{\sim}{X})=\underset{\sim}{X}\left(S^{0}, \ldots, S^{0}\right)$ in $\mathcal{D}$ is called the coefficient spectrum of $\underset{\sim}{X}$. It has the correct homotopy type if $\underset{\sim}{X}$ is multilinear. Given a functor $\underset{\sim}{Y}: \mathcal{S}^{\text {fin }} \rightarrow \mathcal{S}$, Goodwillie calls the coefficient spectrum of the multilinear functor $\operatorname{hocr}_{n} P_{n} \underset{\sim}{Y} \simeq \operatorname{hocr}_{n} D_{n} \underset{\sim}{Y}$ the $n^{\text {th }}$ derivative of $\underset{\sim}{Y}$.

\subsection{Goodwillie's theorem on multilinearized homotopy cross effects}

Proposition 5.28 (Goodwillie [11, Proposition 3.3]) Let $0 \leq m \leq n$. For any $n-$ excisive functor $\underset{\sim}{X}$, the functor hocr $m+1 \underset{\sim}{X}$ is $(n-m)$-excisive in each variable. In particular, the $n^{\text {th }}$ homotopy cross effect is multilinear if $\underset{\sim}{X}$ is $n$-excisive, and it is contractible if $\underset{\sim}{X}$ is $(n-1)$-excisive.

Proof The proof by Goodwillie is again a variation on opaqueness and applies to the setup here.

Definition 5.29 A functor $\underset{\sim}{X}$ is $n$-reduced if $P_{n-1} \underset{\sim}{X} \simeq *$, and $n$-homogeneous if it is $n$-excisive and $n$-reduced.

Definition 5.30 In order to distinguish a functor $\underset{\sim}{X}: \mathcal{C}_{1} \times \cdots \times \mathcal{C}_{n} \rightarrow \mathcal{D}$ in $n$ variables $K_{1}, \ldots, K_{n}$ notationally from the same functor $\underset{\sim}{X}$ when viewed as a functor in one variable $\underline{K}=\left(K_{1}, \ldots, K_{n}\right)$, the latter is denoted $\underset{\sim}{\lambda} \underset{\sim}{X}$.

The $n$-excisive approximation functor $P_{n}$ applies to the functor $\underset{\sim}{X}$. There is a commutative diagram:

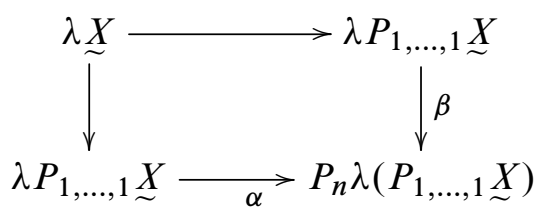

Lemma 5.31 If a functor $\underset{\sim}{X}: \mathcal{C}_{1} \times \cdots \times \mathcal{C}_{n} \rightarrow \mathcal{D}$ is $\left(d_{1}, \ldots, d_{n}\right)$-excisive, then $\underset{\sim}{X}$ is $\left(d_{1}+\cdots+d_{n}\right)$-excisive.

Proof This is [11, Lemma 6.6] whose proof refers to [10, Lemma 3.4]. The proof applies here. 
Lemma 5.32 If a functor $\underset{\sim}{X}: \mathcal{C}_{1} \times \cdots \times \mathcal{C}_{n} \rightarrow \mathcal{D}$ is multireduced, $\lambda \underset{\sim}{X}$ is $n$-reduced.

Proof The proofs of [11, Lemmas 3.2 and 6.7] apply.

Lemma 5.33 If $\underset{\sim}{X}: \mathcal{C}_{1} \times \cdots \times \mathcal{C}_{n} \rightarrow \mathcal{D}$ is multireduced and $\lambda \underset{\sim}{X}$ is $n$-excisive, then $\underset{\sim}{X}$ is multilinear.

Proof The proof of [11, Lemma 6.9] applies here as well.

Corollary 5.34 The maps $\alpha$ and $\beta$ in diagram (5-9) are objectwise weak equivalences for every functor in $\operatorname{Fun}\left(\mathcal{C}_{1} \wedge \cdots \wedge \mathcal{C}_{n}, \mathcal{D}\right)$.

Proof In order to apply the lemmata above, the functor in question is pulled back via $p: \mathcal{C}_{1} \times \cdots \times \mathcal{C}_{n} \rightarrow \mathcal{C}_{1} \wedge \cdots \wedge \mathcal{C}_{n}$. Then the map $\alpha$ is an objectwise weak equivalence by Lemma 5.31. The analogous map

$$
\gamma: P_{n} \lambda \underset{\sim}{X} \rightarrow P_{1, \ldots, 1}\left(P_{n} \lambda \underset{\sim}{X}\right)
$$

is an objectwise weak equivalence by Lemma 5.33. The maps $\beta$ and $\gamma$ are related via a natural weak equivalence commuting the constructions $P_{n}$ and $P_{1, \ldots, 1}$, since homotopy limits, as well as joins, commute among themselves. Hence, $\beta$ is an objectwise weak equivalence as well. The proof finishes by noting that the functor $p^{*}$ detects objectwise weak equivalences, see Lemma 2.23.

Theorem 5.35 (Goodwillie [11, Theorem 6.1]) For all $\mathcal{S}$-functors $\underset{\sim}{X}: \mathcal{C} \rightarrow \mathcal{D}$ there is an natural objectwise weak equivalence under $\operatorname{hocr}_{n} \underset{\sim}{X}$ :

$$
\operatorname{hocr}_{n} P_{n} \underset{\sim}{X} \simeq P_{(1, \ldots, 1)} \operatorname{hocr}_{n} \underset{\sim}{X} \text {. }
$$

Proof Let $\underset{\sim}{X}$ be a functor in $\operatorname{Fun}(\mathcal{C}, \mathcal{D})$. Substituting $\operatorname{hocr}_{n} \underset{\sim}{X}$ in $\operatorname{diagram}(5-9)$ supplies a natural zig-zag of objectwise weak equivalence

$$
P_{1, \ldots, 1} \operatorname{hocr}_{n} \underset{\sim}{X} \simeq P_{n} \lambda\left(\operatorname{hocr}_{n} \underset{\sim}{X}\right)
$$

under hocr ${ }_{n} \underset{\sim}{X}$ by Corollary 5.34. In order to prove that the functors $P_{n} \lambda\left(\operatorname{hocr}_{n} \underset{\sim}{X}\right.$ ) and $\operatorname{hocr}_{n} P_{n} \underset{\sim}{X}$ are naturally weakly equivalent under $\operatorname{hocr}_{n} \underset{\sim}{X}$, denote by $J_{U}(\underset{\sim}{X})$ the functor

$$
\left.K \mapsto J_{U} \underset{\sim}{X}\right)(K)=\underset{\sim}{X}(K \star U) .
$$


for each finite set $U$. The join with $U$ commutes with coproducts in $\mathcal{C}$. Thus, for each $\underline{K}$ in $\mathcal{C}^{\wedge n}$ there is a weak equivalence

$$
\begin{aligned}
J_{U} \lambda\left(\operatorname{hocr}_{n} \underset{\sim}{X}\right)(\underline{K}) & \simeq \operatorname{hofib}\left[\underset{\sim}{X}\left(\bigvee_{i=1}^{n}\left(K_{i} \star U\right)\right) \rightarrow \operatorname{holim}_{S \in P_{0} \underline{\underline{n}}} \underset{\sim}{X}\left(\bigvee_{i \notin S}\left(K_{i} \star U\right)\right)\right] \\
& \simeq \operatorname{hofib}\left[\underset{\sim}{X}\left(\left(\bigvee_{i=1}^{n} K_{i}\right) \star U\right) \rightarrow \operatorname{holim}_{S \in P_{0} \underline{n}} \underset{\sim}{X}\left(\left(\bigvee_{i \notin S} K_{i}\right) \star U\right)\right] \\
& \simeq \operatorname{hocr}_{n} J_{U} \underset{\sim}{X}(\underline{K})
\end{aligned}
$$

and therefore an objectwise weak equivalence $T_{n} \lambda\left(\operatorname{hocr}_{n} \underset{\sim}{X}\right) \simeq \operatorname{hocr}_{n} T_{n} \underset{\sim}{X}$. It induces the desired objectwise weak equivalence $P_{n} \lambda\left(\operatorname{hocr}_{n} \underset{\sim}{X}\right) \simeq \operatorname{hocr}_{n} P_{n} \underset{\sim}{X}$.

Corollary 5.36 The $n^{\text {th }}$ cross effect

$$
\operatorname{cr}_{n}: \operatorname{Fun}(\mathcal{C}, \mathcal{D})_{n-\mathrm{exc}-\mathrm{cr}} \rightarrow \operatorname{Fun}\left(\Sigma_{n} \prec \mathcal{C}^{\wedge n}, \mathcal{D}\right)_{\mathrm{ml}}
$$

is a right Quillen functor.

Proof The left adjoint preserves cofibrations by Lemma 4.25. It suffices to show that $\mathrm{cr}_{n}$ preserves fibrations. Let $f: \underset{\sim}{X} \rightarrow \underset{\sim}{Y}$ be an $n$-excisive $\mathrm{cr}$ fibration, hence an $\mathrm{hf} \mathrm{cr}$ fibration such that the diagram

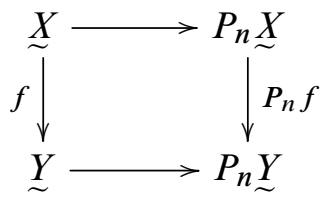

is a homotopy pullback square. Lemma 4.25 implies that the map $\mathrm{cr}_{n} f$ is an hf fibration. It remains to check that the diagram

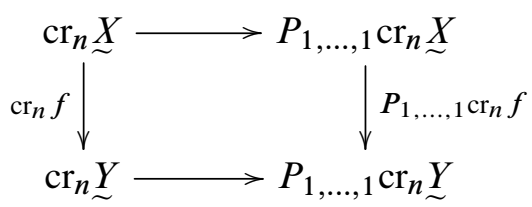

is a homotopy pullback. This square is the front of a commutative cube, whose sides are induced by the natural map $\operatorname{cr}_{n} \underset{\sim}{X} \rightarrow \operatorname{hocr}_{n} \underset{\sim}{X}$. The back of the cube is the following diagram:

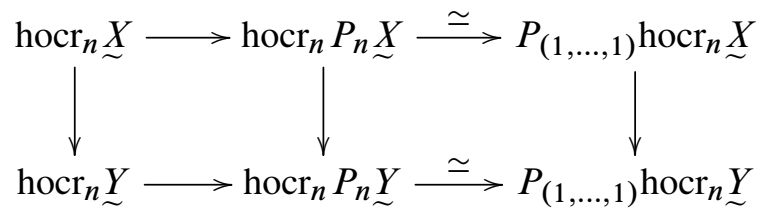


The horizontal maps on the right are objectwise weak equivalences by Goodwillie's Theorem 5.35. The square on the left-hand side is the image of a homotopy pullback square under $\operatorname{hocr}_{n}$. Thus, $\mathrm{cr}_{n} f$ is a multilinear fibration, once the sides of the commutative cube are proven to be homotopy pullback squares as well. In fact, it suffices to check that the square

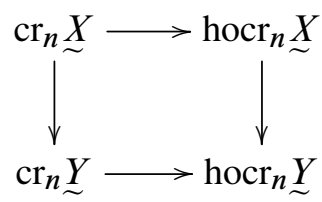

is a homotopy pullback square, because the opposite side of the cube is obtained by applying $P_{1, \ldots, 1}$ and inherits the homotopy pullback property. Let $\underset{\sim}{F}$ be the fiber of $f$. It is cr fibrant, thus by Lemma 3.24 the canonical map

$$
\mathrm{cr}_{n} \underset{\sim}{F} \rightarrow \operatorname{hocr}_{n} \underset{\sim}{F}
$$

of vertical (homotopy) fibers in diagram (5-10) is an objectwise weak equivalence. As the multilinear model structure is stable by Corollary 5.25, diagram (5-10) is a homotopy pullback square, which completes the proof.

\section{Homogeneous functors}

As recalled in Definition 5.29, a functor $\underset{\sim}{X}: \mathcal{C} \rightarrow \mathcal{D}$ is $n$-homogeneous if it is $n-$ excisive and $P_{n-1} \underset{\sim}{X}$ is contractible. In this section, $\operatorname{Fun}(\mathcal{C}, \mathcal{D})$ will be equipped via right Bousfield localization with a model structure in which every functor is weakly equivalent to an $n$-homogeneous functor. As shown in Theorem 6.10, this model category is Quillen equivalent to the multilinear model structure on $\operatorname{Fun}\left(\Sigma_{n} \prec \mathcal{C}^{\wedge n}, \mathcal{D}\right)$. Hence the $n$-homogeneous model category is also Quillen equivalent, by Theorem 5.27, to the model category of $\Sigma_{n}$-spectra in $\mathcal{D}$, provided $\mathcal{C}$ is the category of finite pointed simplicial sets. This yields a construction of derivatives for functors in $\operatorname{Fun}\left(\mathcal{S}^{\text {fin }}, \mathcal{D}\right)$ on the level of model categories.

Convention 6.1 Suppose in addition to Convention 5.2 that $\mathcal{D}$ admits a set of generating cofibrations with cofibrant domains.

\subsection{The homogeneous model structure}

Definition 6.2 Consider the following set of objects of $\operatorname{Fun}(\mathcal{C}, \mathcal{S})$ :

$$
\Lambda_{n}=\left\{\bigwedge_{i=1}^{n} R^{K_{i}} \mid K_{1}, \ldots, K_{n} \in \mathrm{Ob}(\mathcal{C})\right\} .
$$


More generally, let $I_{\mathcal{D}}$ be a set of generating cofibrations with cofibrant domains in $\mathcal{D}$, which exists by Convention 6.1. Let $\operatorname{cd}\left(I_{\mathcal{D}}\right)$ denote the set of domains and codomains of all morphisms $i \in I$, and set

$$
\Lambda_{n, I_{\mathcal{D}}}=\left\{\left(R^{K_{1}} \wedge \cdots \wedge R^{K_{n}}\right) \wedge D \mid K_{1}, \ldots, K_{n} \in \mathrm{Ob}(\mathcal{C}), D \in \operatorname{cd}\left(I_{\mathcal{D}}\right)\right\}
$$

A map $f$ in $\operatorname{Fun}(\mathcal{C}, \mathcal{D})_{n \text {-exc-cr }}$ is:

(1) An $n$-homogeneous equivalence if it is a $\Lambda_{n, I_{\mathcal{D}}}$-colocal equivalence.

(2) An $n$-homogeneous cofibration if it has the left lifting property with respect to all $n$-excisive cr fibrations that are also $n$-homogeneous equivalences.

The $n$-homogeneous fibrations are the $n$-excisive cr fibrations.

The notion of colocal equivalence is taken from Hirschhorn [12, 3.1.4(b)]. Choosing a different set of generating cofibrations with cofibrant domains in $\mathcal{D}$ yields the same classes, due to the following well-known lemma, whence the choice of generating cofibrations of $\mathcal{D}$ will be omitted from the notation.

Lemma 6.3 Let $\mathcal{D}$ be an $\mathcal{S}$-model category and $f$ a morphism of fibrant objects. Suppose $\mathcal{D}$ admits a set $I_{\mathcal{D}}$ of generating cofibrations with cofibrant domains. The morphism $f$ is a weak equivalence if and only if for every domain and every codomain $D$ appearing in $\operatorname{cd}\left(I_{\mathcal{D}}\right)$, the map $\mathcal{D}(D, f)$ is a weak equivalence of simplicial sets.

Theorem 6.4 Assume Convention 6.1. The classes described in Definition 6.2 form a right proper $\mathcal{S}$-model structure on $\operatorname{Fun}(\mathcal{C}, \mathcal{D})$.

Proof This follows from Christensen and Isaksen [6, Theorem 2.6], which applies to any cofibrantly generated right proper model category. In our case this is the $n$-excisive model structure on $\operatorname{Fun}(\mathcal{C}, \mathcal{D})$.

The right Bousfield localization of the $n$-excisive cross effect model structure on the category $\operatorname{Fun}(\mathcal{C}, \mathcal{D})$ with respect to the set $\Lambda_{n, I_{\mathcal{D}}}$ is the $n$-homogeneous model structure and is denoted by $\operatorname{Fun}(\mathcal{C}, \mathcal{D})_{n \text {-hom }}$. Theorem 5.23 implies that the $n$-homogeneous model structure on $\operatorname{Fun}(\mathcal{C}, \operatorname{Sp}(\mathcal{D}))$ exists.

Definition 6.5 The $n$-homogeneous part of a functor $\underset{\sim}{X}$ is defined as

$$
D_{n} \underset{\sim}{X}:=\operatorname{hofib}\left[q_{n}: P_{n} \underset{\sim}{X} \rightarrow P_{n-1} \underset{\sim}{X}\right] .
$$

By construction, $D_{n} \underset{\sim}{X}$ is indeed $n$-homogeneous. 
Lemma 6.6 For every functor $\underset{\sim}{X}$ the map $D_{n} \underset{\sim}{X} \rightarrow P_{n} \underset{\sim}{X}$ induces an objectwise equivalence $\operatorname{hocr}_{n} D_{n} \underset{\sim}{X} \rightarrow \operatorname{hocr}_{n} \widetilde{\widetilde{P}}_{n} \underset{\sim}{X}$.

Proof Proposition 5.28 implies that $\operatorname{hocr}_{n} P_{n-1} \underset{\sim}{X} \simeq *$. The chain

$$
\begin{aligned}
\operatorname{hocr}_{n} D_{n} \underset{\sim}{X} & \simeq \operatorname{hocr}_{n} \operatorname{hofib}\left[P_{n} \underset{\sim}{X} \rightarrow P_{n-1} \underset{\sim}{X}\right] \\
& \simeq \operatorname{hofib}\left[\operatorname{hocr}_{n} P_{n} \underset{\sim}{X} \rightarrow \operatorname{hocr}_{n} P_{n-1} \underset{\sim}{X}\right] \simeq \operatorname{hocr}_{n} P_{n} \underset{\sim}{X}
\end{aligned}
$$

of natural objectwise equivalences completes the proof.

Lemma 6.7 For $f$ in $\operatorname{Fun}(\mathcal{C}, \mathcal{D})$ the following statements are equivalent:

(1) The map $f$ is an $n$-homogeneous equivalence.

(2) The induced map $\operatorname{hocr}_{n} P_{n}(f)$ is an objectwise equivalence.

(3) The induced map $\operatorname{hocr}_{n} D_{n}(f)$ is an objectwise equivalence.

(4) The induced map $\operatorname{hocr}_{n}(f)$ is a multilinear equivalence.

Proof A map $f: \underset{\sim}{X} \rightarrow \underset{\sim}{Y}$ is an $n$-homogeneous equivalence if and only if an appropriate map between cr fibrant approximations is one. Assume that $\underset{\sim}{X}$ and $\underset{\sim}{Y}$ are cr fibrant. Lemma 6.3 implies that $f$ is an $n$-homogeneous equivalence if and only if for every $D$ in $\operatorname{cd}\left(I_{D}\right)$ the induced map

$$
\mathcal{S}_{\text {Fun }(\mathcal{C D})}\left(\left(\bigwedge_{i=1}^{n} R^{K_{i}}\right) \wedge D, P_{n}(f)\right) \cong \mathcal{S}_{\mathcal{D}}\left(D, \operatorname{cr}_{n} P_{n}(f)\left(K_{1}, \ldots, K_{n}\right)\right)
$$

is a weak equivalence. Since $\underset{\sim}{X}$ is cr fibrant, Lemma 3.24 and Theorem 5.35 entail that

$$
\operatorname{cr}_{n} P_{n} \underset{\sim}{X} \simeq \operatorname{hocr}_{n} P_{n} \underset{\sim}{X} \simeq P_{1, \ldots, 1} \operatorname{hocr}_{n} \underset{\sim}{X}
$$

and similarly for $\underset{\sim}{Y}$. This shows that assertion (1), (2) and (4) are equivalent to each other if one observes, that $\operatorname{hocr}_{n} P_{n}(f)$ is a multilinear equivalence between multilinear functors by Proposition 5.28. Statements (2) and (3) are equivalent by Lemma 6.6.

Corollary 6.8 Every functor $\underset{\sim}{X}$ is $n$-homogeneously equivalent to $D_{n} \underset{\sim}{X}$.

Proof The map $\underset{\sim}{X} \rightarrow P_{n} \underset{\sim}{X}$ is an $n$-excisive equivalence, hence an $n$-homogeneous equivalence. By Lemma 6.7 and Lemma 6.6, the map $D_{n} \underset{\sim}{X} \rightarrow P_{n} \underset{\sim}{X}$ is an $n-$ homogeneous equivalence.

The next statement also holds for functors to an unstable category $\mathcal{D}$, but it will be shown later in Lemma 6.19, after some auxiliary statements.

Corollary 6.9 A map $f$ in $\operatorname{Fun}(\mathcal{C}, \operatorname{Sp}(\mathcal{D}))$ is an $n$-homogeneous equivalence if and only if the induced map $D_{n}(f)$ is an objectwise equivalence. 
Proof By Corollary 6.8, it remains to show that $D_{n}(f)$ is an objectwise equivalence if and only if $\operatorname{hocr}_{n} D_{n}(f)$ is an objectwise equivalence. This is the content of [11, Proposition 3.4] where Goodwillie actually shows that a functor to $\operatorname{Sp}(\mathcal{D})$ is $(n-1)-$ excisive if it is $n$-excisive with contractible $n^{\text {th }}$ homotopy cross effect. In the proof, the following two properties are used:

(1) Every strongly homotopy cocartesian cube of cofibrant objects admits a weak equivalence from a pushout cube (see Goodwillie [10, Proposition 2.2]). This holds in every model category.

(2) If a map $\mathcal{X} \rightarrow \mathcal{Y}$ of $n$-cubes is homotopy Cartesian as an $(n+1)$-cube and $\mathcal{Y}$ is homotopy Cartesian, then $\mathcal{X}$ is homotopy Cartesian. This holds in every model category.

This implies the statement for stable model categories, because there a map is a weak equivalence if and only if its homotopy fiber is contractible.

Theorem 6.10 Assume Convention 6.1. The functors

$$
\mathcal{L}_{n}: \operatorname{Fun}\left(\Sigma_{n} \prec \mathcal{C}^{\wedge n}, \operatorname{Sp}(\mathcal{D})\right)_{\mathrm{ml}} \rightleftarrows \operatorname{Fun}(\mathcal{C}, \operatorname{Sp}(\mathcal{D}))_{n-\text { hom }}: \mathrm{cr}_{n}
$$

form a Quillen equivalence.

Proof The $n^{\text {th }}$ cross effect is a right Quillen functor from the $n$-excisive cr model structure by Corollary 5.36. In particular, $\mathrm{cr}_{n}$ preserves fibrations. Lemma 3.24 and Lemma 6.7 show that $\mathrm{cr}_{n}$ preserves and detects $n$-homogeneous equivalences on cr fibrant objects. Hence if $p$ is an acyclic fibration in the $n$-homogeneous $\mathrm{cr}$ model structure with fiber $\underset{\sim}{F}$, the map $\operatorname{cr}_{n}(p)$ is a fibration with contractible fiber $\operatorname{cr}_{n}(\underset{\sim}{F})$. As the multilinear model structure is stable by Corollary $5.25, \operatorname{cr}_{n}(p)$ is an acyclic fibration. Thus $\mathrm{cr}_{n}$ is a right Quillen functor on the $n$-homogeneous model structure. The argument from [11, page 678] extends to show that the derived unit map $\underset{\sim}{X} \rightarrow \operatorname{hocr}_{n} \mathcal{L}_{n} \underset{\sim}{X}$ is an equivalence. The already mentioned fact that $\mathrm{cr}_{n}$ detects $n-$ homogeneous equivalences on cr fibrant objects implies it is a Quillen equivalence.

\subsection{Goodwillie's delooping theorem}

Theorem 6.11 Suppose that $\mathcal{C}$ and $\mathcal{D}$ satisfy Convention 6.1. The pair of adjoint functors obtained by composing with the functors

$$
\operatorname{Fr}_{0}: \mathcal{D} \rightarrow \operatorname{Sp}(\mathcal{D}) \quad \text { and } \quad \operatorname{Ev}_{0}: \operatorname{Sp}(\mathcal{D}) \rightarrow \mathcal{D}
$$

is a Quillen equivalence:

$$
F: \operatorname{Fun}(\mathcal{C}, \mathcal{D})_{n-\text { hom }} \rightleftarrows \operatorname{Fun}(\mathcal{C}, \operatorname{Sp}(\mathcal{D}))_{n \text {-hom }}: G
$$


Proof The proof is divided into several steps. The pair is a Quillen adjunction by Lemma 6.12. The total right derived functor of $G$ is faithful by Lemma 6.13, and essentially surjective and full by Lemma 6.16 .

Lemma 6.12 The functors $(F, G)$ form a Quillen pair for the $n$-homogeneous model structures on both sides.

Proof The functor $F$ maps the generating sets $I^{\mathrm{cr}}$ and $J^{\mathrm{cr}}$ on the left-hand side into the corresponding ones on the right-hand side. This implies that $F$ is a left Quillen functor on cr model structures. The functor $\mathrm{Ev}_{0}$ commutes with all colimits, limits and homotopy limits. In particular, it commutes with the functors $(-)^{\text {hf }}$ and $P_{n}$ up to natural weak equivalence. Thus, the characterization of fibrations in the hf model structure in Lemma 4.15 and in the $n$-excisive model structure in Definition 5.7 yields that $G$ is a right Quillen functor on hf model structures and the $n$-excisive model structure. The acyclic fibrations agree in all these model structures.

The fibrations in the $n$-homogeneous and the $n$-excisive model structure agree. Suppose that $f$ is an $n$-homogeneous acyclic fibration. Since $\mathrm{Ev}_{0}$ commutes with $P_{n}$ and with homotopy fibers, we have $\operatorname{hocr}_{n} P_{n} G(f) \simeq G\left(\operatorname{hocr}_{n} P_{n} f\right)$. It follows from Lemma 6.7 that $G(f)$ is an $n$-homogeneous acyclic fibration, whence $G$ is a right Quillen functor also for the $n$-homogeneous model structure. Alternatively, the left adjoint $F$ preserves the set of objects that define the right Bousfield localization.

Lemma 6.13 The functor $G$ preserves and detects $n$-homogeneous equivalences of bifibrant functors, and its total right derived functor is faithful on morphisms.

Proof General localization theory of model categories implies that a map between $n$-homogeneously bifibrant functors is an $n$-homogeneous equivalence if and only if it is an objectwise equivalence. The functor $\mathrm{Ev}_{0}$ preserves and detects weak equivalences of stably fibrant spectra. Thus, the functor $G$ preserves and detects $n$-homogeneous equivalences of bifibrant functors.

Moreover, the structure maps $\underset{\sim}{X} \rightarrow \Omega \underset{\sim}{X}$ of an $n$-homogeneously bifibrant functor $\underset{\sim}{X}: \mathcal{C} \rightarrow \operatorname{Sp}(\mathcal{D})$ are objectwise weak equivalences. Hence, if $f$ and $g$ are maps of bifibrant objects in the $n$-homogeneous model structure on $\operatorname{Sp}(\mathcal{D})$-valued functors such that $f_{0}$ and $g_{0}$ are homotopic, then $f$ and $g$ are homotopic. In particular, the total right derived functor of $G$ is faithful on morphisms.

For the next lemma recall that all $\mathcal{S}$-functors are reduced. 
Lemma 6.14 Let $n>0$ and $\underset{\sim}{X}$ a functor. Then there is a natural commutative diagram

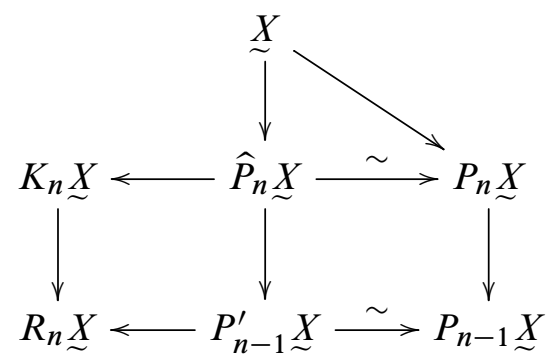

in which the left-hand square is a homotopy pullback square, the functor $K_{n} \underset{\sim}{X}$ is contractible and the functor $R_{n} \underset{\sim}{X}$ is $n$-homogeneous.

Proof The diagram above is diagram (2.3) in [11] and Goodwillie's proof of its existence in Section 2 of that article applies.

Lemma 6.15 Let $\underset{\sim}{X}: \mathcal{C} \rightarrow \mathcal{D}$ be an $n$-reduced functor. Then there exists a spectrumvalued functor $\mathbf{R}_{n} \underset{\sim}{\widetilde{X}}: \mathcal{C} \rightarrow \operatorname{Sp}(\mathcal{D})$ and a natural objectwise equivalence $G\left(\mathbf{R}_{n} \underset{\sim}{X}\right) \simeq \underset{\sim}{X}$.

Proof If a functor $\underset{\sim}{X}: \mathcal{C} \rightarrow \mathcal{D}$ is $n$-reduced, not only the upper left corner $K_{n} \underset{\sim}{X}$, but also the lower right corner $P_{n-1} \underset{\sim}{X}$ of the homotopy pullback square from Lemma 6.14 is contractible. Let $U_{n} \underset{\sim}{X}$ denote the homotopy pullback of that square. The natural objectwise weak equivalences

$$
\underset{\sim}{X} \stackrel{\simeq}{\longrightarrow} \hat{P}_{n} \underset{\sim}{X} \stackrel{\simeq}{\longrightarrow} U_{n} \underset{\sim}{X} \stackrel{\simeq}{\longleftarrow} R_{n} \underset{\sim}{X},
$$

do not form a direct map which can be iterated to obtain a spectrum-valued functor $\mathbf{R}_{n} \underset{\sim}{X}$. However, a trick by Goodwillie [9] given at the end of the introduction works. For $j \geq 0$, let $\left(\mathbf{R}_{n} \underset{\sim}{X}\right)_{j}$ denote the homotopy limit (not colimit) of the diagram

$$
R_{n}^{j} \underset{\sim}{\longrightarrow} \stackrel{\simeq}{\longrightarrow} U\left(R_{n}^{j} \underset{\sim}{X}\right) \stackrel{\simeq}{\longleftarrow} \Omega R_{n}^{j+1} \underset{\sim}{\longrightarrow} \stackrel{\simeq}{\longrightarrow} \Omega U\left(R_{n}^{j+1} \underset{\sim}{X}\right) \stackrel{\simeq}{\longleftarrow} \Omega^{2} R_{n}^{j+2} \underset{\sim}{X} \stackrel{\simeq}{\longrightarrow} \cdots,
$$

starting with $R_{n}^{0} \underset{\sim}{X}=\underset{\sim}{X}$. Then $\left(\mathbf{R}_{n} \underset{\sim}{X}\right)_{j} \stackrel{\simeq}{\longrightarrow} R_{n}^{j} \underset{\sim}{X}$ for all $j \geq 0$, and there are structure maps

$$
\left(\mathbf{R}_{n} \underset{\sim}{X}\right)_{j} \stackrel{\simeq}{\longrightarrow} \Omega\left(\mathbf{R}_{n} \underset{\sim}{X}\right)_{j+1}
$$

defining a spectrum-valued functor $\mathbf{R}_{n} \underset{\sim}{X}$ such that $G\left(\mathbf{R}_{n} \underset{\sim}{X}\right) \simeq \underset{\sim}{X}$.

Lemma 6.16 The total right derived functor of $G$ is essentially surjective and full.

Proof By Corollary 6.8, any functor in $\operatorname{Fun}(\mathcal{C}, \mathcal{D})$ is $n$-homogeneously equivalent to an $n$-homogeneous functor $\underset{\sim}{X}$. Lemma 6.15 supplies a functor $\mathbf{R}_{n} \underset{\sim}{X}$ with $G\left(\mathbf{R}_{n} \underset{\sim}{X}\right) \simeq$ $\underset{\sim}{X}$. The statement follows. 


\subsection{The Quillen equivalences}

There is a commutative diagram of Quillen pairs:

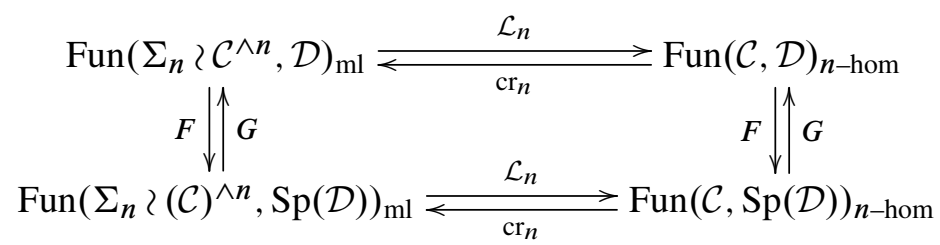

The left vertical Quillen pair was shown to be a Quillen equivalence in Theorem 5.24, the lower horizontal one in Theorem 6.10, and the right vertical pair in Theorem 6.11. The 2-out-of-3 property of Quillen equivalences yields the following statement.

Corollary 6.17 Suppose Convention 6.1 holds. Then the pair

$$
\mathcal{L}_{n}: \operatorname{Fun}\left(\Sigma_{n} \prec \mathcal{C}^{\wedge n}, \mathcal{D}\right)_{\mathrm{ml}} \rightleftarrows \operatorname{Fun}(\mathcal{C}, \mathcal{D})_{n-\text { hom }}: \mathrm{cr}_{n}
$$

is a Quillen equivalence.

The associated diagram of total derived functors on homotopy categories yields Goodwillie's diagram of equivalences of homotopy categories (as displayed in the introduction). If $\mathcal{C}=\mathcal{S}^{\text {fin }}$, evaluation at $S^{0}$ prolongs this Quillen equivalence to the category of $\Sigma_{n}$-spectra in $\mathcal{D}$, by Theorem 5.27.

Corollary 6.18 The $n$-homogeneous model structure on $\operatorname{Fun}(\mathcal{C}, \mathcal{D})$ is stable, if Convention 6.1 holds.

\subsection{More on the homogeneous model structures}

The following assertion was proved for $\operatorname{Sp}(\mathcal{D})$ as target category already in Corollary 6.9. The validity of Convention 6.1 is assumed for the remainder of this article.

Lemma 6.19 A map $f$ in $\operatorname{Fun}(\mathcal{C}, \mathcal{D})$ is an $n$-homogeneous equivalence if and only if the induced map $D_{n}(f)$ is an objectwise weak equivalence.

Proof By Lemma 6.7 and Theorem 5.35, the map $f$ is an $n$-homogeneous equivalence if and only if $\operatorname{hocr}_{n} P_{n}(f) \simeq \operatorname{hocr}_{n} D_{n}(f)$ is an objectwise equivalence. This shows immediately that if $D_{n}(f)$ is an objectwise equivalence, then $f$ is an $n$-homogeneous equivalence.

Conversely, let $f$ be an $n$-homogeneous equivalence. By Corollary 6.8 it can be replaced by the induced $D_{n}(f): D_{n} \underset{\sim}{X} \rightarrow D_{n} \underset{\sim}{Y}$. Lemma 6.15 states that there exists 
$n$-homogeneous functors $\mathbf{R}_{n} \underset{\sim}{X}$ and $\mathbf{R}_{n} \underset{\sim}{Y}$ to $\operatorname{Sp}(\mathcal{D})$ and a map $g=\mathbf{R}_{n}(f)$ between them such that $G(g)=D_{n}(f)$. The functor $G$ detects $n$-homogeneous equivalences by Lemma 6.13 . Thus, $g$ is an $n$-homogeneous equivalence. Corollary 6.9 shows that $g=D_{n}(g)$ is an objectwise equivalence of symmetric multilinear functors to $\operatorname{Sp}(\mathcal{D})$. Hence, $G(g)=D_{n}(f)$ is an objectwise weak equivalence.

Corollary 6.20 For any functor $\underset{\sim}{X}: \mathcal{C} \rightarrow \mathcal{D}$ and $n>0$ there exists a functor $R_{n} \underset{\sim}{X}: \mathcal{C} \rightarrow$ $\mathcal{D}$ and a zig-zag of natural weak equivalences $D_{n} \underset{\sim}{X} \simeq \Omega R_{n} \underset{\sim}{X}$.

Proof This follows from Lemma 6.14.

Corollary 6.21 Let $n>0$. A map $f: \underset{\sim}{X} \rightarrow \underset{\sim}{Y}$ in $\operatorname{Fun}(\mathcal{C}, \mathcal{D})$ induces an objectwise weak equivalence $D_{n}(f)$ if and only if the diagram

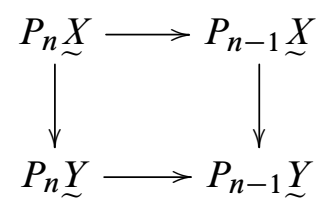

is an objectwise homotopy pullback square.

Proof If the diagram is a homotopy pullback square, then its homotopy fibers are weakly equivalent via $D_{n}(f)$. For the converse note that $R_{n}(f)$ is a weak equivalence if $D_{n}(f)$ is by Corollary 6.20. The horizontal maps are part of homotopy fiber sequences

$$
P_{n} \underset{\sim}{X} \rightarrow P_{n-1} \underset{\sim}{X} \rightarrow R_{n} \underset{\sim}{X} \quad \text { and } \quad P_{n} \underset{\sim}{Y} \rightarrow P_{n-1} \underset{\sim}{Y} \rightarrow R_{n} \underset{\sim}{Y}
$$

by Lemma 6.14. So the square is a homotopy pullback.

Lemma 6.22 A map is an $n$-homogeneous acyclic fibration if and only if it is an $(n-1)$-excisive cr fibration and an $n$-homogeneous equivalence.

Proof By Definition 5.7, a map is an (n-1)-excisive cr fibration if and only if it is a fibration $f: \underset{\sim}{X} \rightarrow \underset{\sim}{Y}$ in the hf cr model structure that induces the following objectwise homotopy pullback diagram:

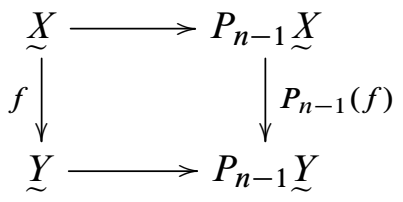


Then it follows that in the diagram

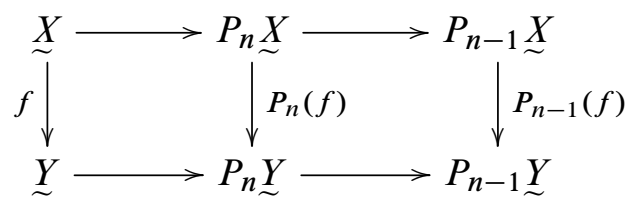

the outer square and the right-hand square are homotopy pullbacks. Therefore, the left-hand square is a homotopy pullback, and $f$ is an $n$-excisive cr fibration which is the same as an $n$-homogeneous fibration. Because it is an $n$-homogeneous equivalence by assumption, it is an $n$-homogeneous acyclic fibration.

Suppose now that $f$ is an $n$-homogeneous acyclic fibration. An $n$-homogeneous fibration is the same as an $n$-excisive cr fibration, hence the left-hand square of diagram (6-4) is a homotopy pullback. Because (6-3) is also a homotopy pullback, the combined outer square is so. Hence, $p$ is an $(n-1)$-excisive cr fibration. It is an $n$-homogeneous equivalence by assumption.

Lemma 6.23 Let $\underset{\sim}{X}$ and $\underset{\sim}{Y}$ be $n$-excisively cr fibrant. Then a map $p: \underset{\sim}{X} \rightarrow \underset{\sim}{Y}$ is an $n$-homogeneous acyclic fibration if and only if it is an (n-1)-excisive cr fibration.

Proof According to Lemma 6.22, it suffices to show that an (n-1)-excisive cr fibration between $n$-excisively cr fibrant objects is already an $n$-homogeneous equivalence. In this case the outer square of diagram (6-4) is a homotopy pullback and the horizontal maps in the left-hand square are weak equivalences. Thus, the square on the right-hand side is a homotopy pullback square, and the induced map of fibers $D_{n} \underset{\sim}{X} \rightarrow D_{n} \underset{\sim}{Y}$ is a weak equivalence.

Lemma 6.24 A cr cofibration is an $n$-homogeneous cofibration if and only if it is $(n-1)$-excisive equivalence.

Proof Right properness implies that a map is an acyclic cofibration in the $n$-homogeneous model structure if and only if it has the left lifting property with respect to all $n$-homogeneous fibrations between fibrant objects (see Hirschhorn [12, Proposition 13.2.1]). The fibrant objects are exactly the $n$-excisively cr fibrant functors. Thus, the stated equivalence follows from Lemma 6.23.

Corollary 6.25 A functor is cofibrant in the $n$-homogeneous model structure if and only if it is cr cofibrant and $n$-reduced.

Proof This follows from Lemma 6.24. 
In particular, one can view the $n$-homogeneous model structure as the "fiber" model structure of the left Quillen functor

$$
\text { Id: } \operatorname{Fun}(\mathcal{C}, \mathcal{D})_{n \text {-exc-cr }} \rightarrow \operatorname{Fun}(\mathcal{C}, \mathcal{D})_{(n-1) \text {-exc-cr }}
$$

of excisive model structures. This supplies a concrete instance where the homotopy fiber model category of Bergner [2, Theorem 3.1] exists.

\section{References}

[1] D Barnes, P Oman, Model categories for orthogonal calculus, Algebr. Geom. Topol. 13 (2013) 959-999 MR3044598

[2] J E Bergner, Homotopy fiber products of homotopy theories, Israel J. Math. 185 (2011) 389-411 MR2837143

[3] G Biedermann, B Chorny, O Röndigs, Calculus of functors and model categories, Adv. Math. 214 (2007) 92-115 MR2348024

[4] F Borceux, Handbook of categorical algebra 2: Categories and structures, Encycl. Math. Appl. 51, Cambridge Univ. Press (1994) MR1313497

[5] A K Bousfield, On the telescopic homotopy theory of spaces, Trans. Amer. Math. Soc. 353 (2001) 2391-2426 MR1814075

[6] J D Christensen, D C Isaksen, Duality and pro-spectra, Algebr. Geom. Topol. 4 (2004) 781-812 MR2100680

[7] B I Dundas, O Röndigs, P A Østvær, Enriched functors and stable homotopy theory, Doc. Math. 8 (2003) 409-488 MR2029170

[8] S Eilenberg, G M Kelly, Closed categories, from: "Proc. Conf. Categorical Algebra", (S Eilenberg, D K Harrison, S Mac Lane, H Röhri, editors), Springer, New York (1966) 421-562 MR0225841

[9] T G Goodwillie, Calculus I: The first derivative of pseudoisotopy theory, $K$-Theory 4 (1990) 1-27 MR1076523

[10] T G Goodwillie, Calculus II: Analytic functors, K-Theory 5 (1991/92) 295-332 MR1162445

[11] T G Goodwillie, Calculus III: Taylor series, Geom. Topol. 7 (2003) 645-711 MR2026544

[12] PS Hirschhorn, Model categories and their localizations, Mathematical Surveys and Monographs 99, Amer. Math. Soc. (2003) MR1944041

[13] M Hovey, Model categories, Mathematical Surveys and Monographs 63, Amer. Math. Soc. (1999) MR1650134 
[14] D C Isaksen, Flasque model structures for simplicial presheaves, K-Theory 36 (2005) 371-395 MR2275013

[15] G M Kelly, Basic concepts of enriched category theory, London Math. Soc. Lecture Note Series 64, Cambridge Univ. Press (1982) MR651714

[16] N J Kuhn, Goodwillie towers and chromatic homotopy: an overview, from: "Proceedings of the Nishida Fest", (M Ando, N Minami, J Morava, W S Wilson, editors), Geom. Topol. Monogr. 10 (2007) 245-279 MR2402789

[17] J Lurie, Higher algebra (2012)

[18] M Lykadis, Simplicial functors and stable homotopy theory, 98-049, SFB 343 Bielefeld (1998) Available at http://www.mathematik.uni-bielefeld.de/sfb343/ preprints/index $98 . \mathrm{html}$

[19] L Pereira, A general context for Goodwillie calculus arXiv:1301.2832

[20] C Rezk, A streamlined proof of Goodwillie's n-excisive approximation, Algebr. Geom. Topol. 13 (2013) 1049-1051 MR3044601

[21] S Schwede, Spectra in model categories and applications to the algebraic cotangent complex, J. Pure Appl. Algebra 120 (1997) 77-104 MR1466099

[22] F Waldhausen, Algebraic K-theory of spaces, from: "Algebraic and geometric topology", (A Ranicki, N Levitt, F Quinn, editors), Lecture Notes in Math. 1126, Springer, Berlin (1985) 318-419 MR802796

Institut für Mathematik, Universität Osnabrück

D-49069 Osnabrück, Germany

gbiederm@uni-osnabrueck.de, oroendig@uni-osnabrueck.de

Received: 12 August 2013 Revised: 31 December 2013 
